Aus der Poliklinik für Zahnärztliche Prothetik

(Prof. Dr. med. dent. R. Bürgers)

im Zentrum Zahn-, Mund- und Kieferheilkunde

der Medizinischen Fakultät der Universität Göttingen

\title{
Langzeitevaluation zur klinischen Bewährung von Resilienzteleskopprothesen
}

\author{
INAUGURAL-DISSERTATION \\ zur Erlangung des Doktorgrades \\ für Zahnheilkunde \\ der Medizinischen Fakultät der \\ Georg-August-Universität zu Göttingen \\ vorgelegt von \\ Vicky Wiedemann \\ aus \\ Chemnitz
}

Göttingen 2017 
Dekan:

Referent:

Ko-Referent:
Prof. Dr. rer. nat. H. K. Kroemer PD Dr. med. dent. Matthias Rödiger Prof. Dr. med. dent. Michael Hülsmann

Datum der mündlichen Prüfung: 12.04.2018 
Hiermit erkläre ich, die Dissertation mit dem Titel "Langzeitevaluation zur klinischen Bewährung von Resilienzteleskopprothesen“ eigenständig angefertigt und keine anderen als die von mir angegebenen Quellen und Hilfsmittel verwendet zu haben.

Göttingen, den 25.10.2017 


\section{Inhaltsverzeichnis}

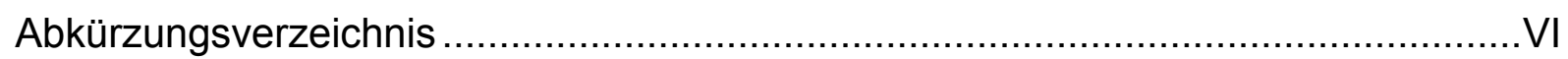

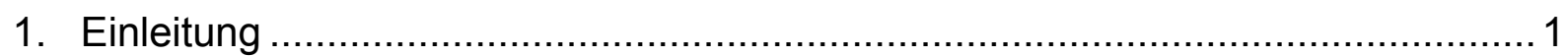

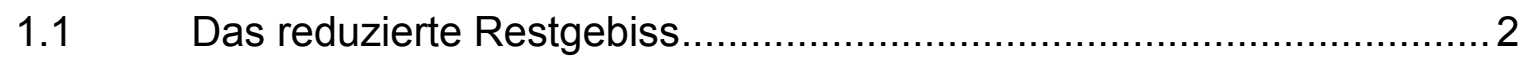

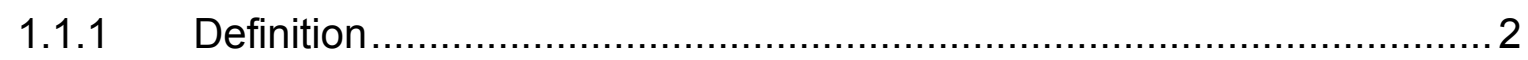

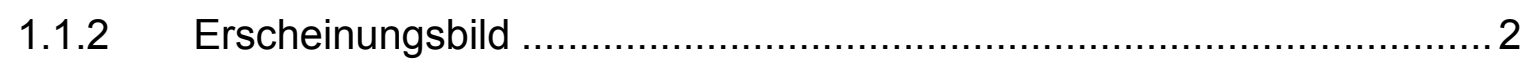

1.1.3 Die Notwendigkeit für den Erhalt von Restzähnen ...............................2

1.2 Doppelkronen .......................................................................

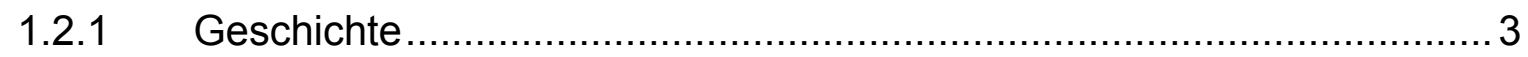

1.2.2 Definition der Doppelkronen .................................................. 4

1.2.3 Klassifikation und Auswahlschema zur Therapie ................................ 4

1.2.4 Doppelkronen als Elemente der starren Lagerung ….......................... 6

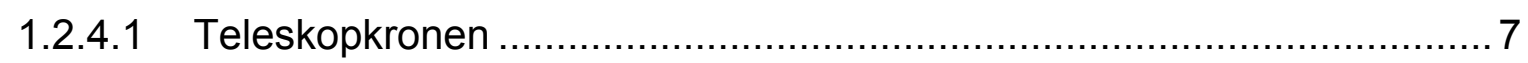

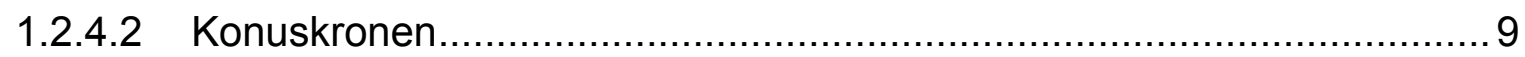

1.2.4.3 Doppelkronen mit Halteelement ..................................................

1.2.5 Doppelkronen als Elemente der beweglichen Lagerung bei

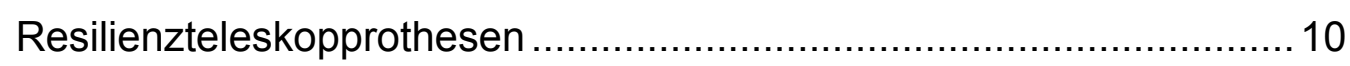

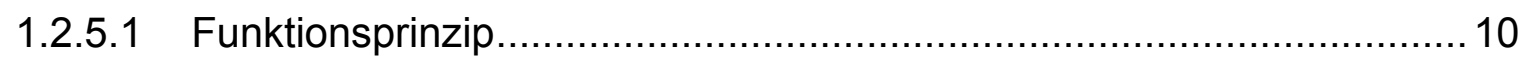

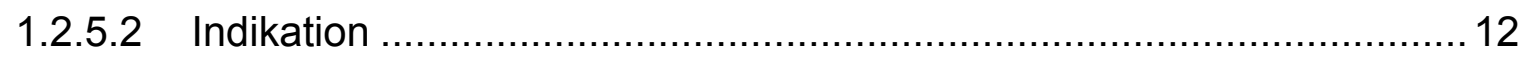

1.2.5.3 Bewährung von Resilienzteleskopprothesen im stark reduzierten

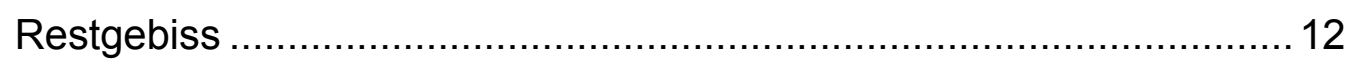

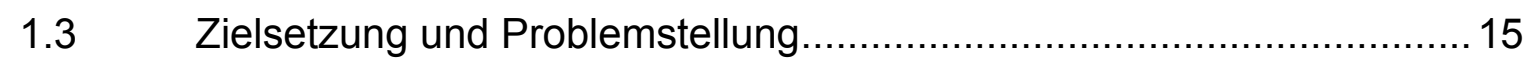

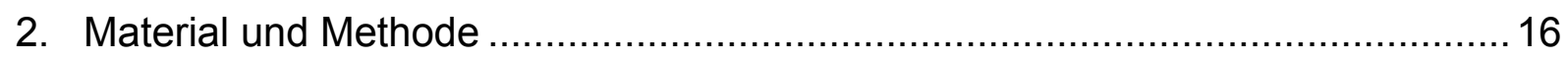

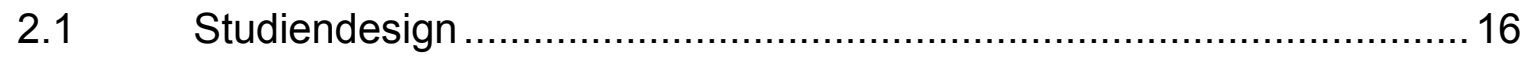

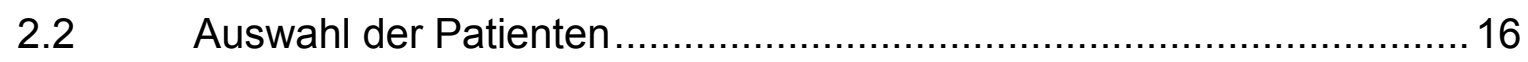

$2.3 \quad$ Untersuchte Parameter/Auswertung .................................................. 17

$2.4 \quad$ Art der Dokumentation................................................................ 19

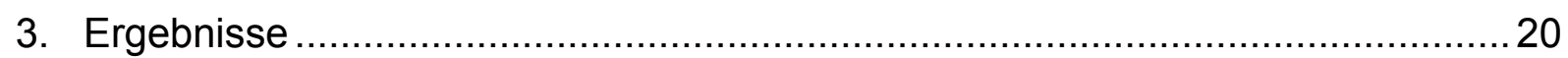

3.1 Häufigkeitsverteilung verschiedener Parameter ............................... 20

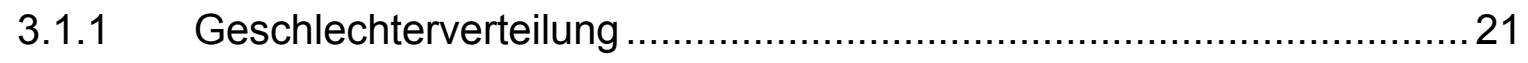

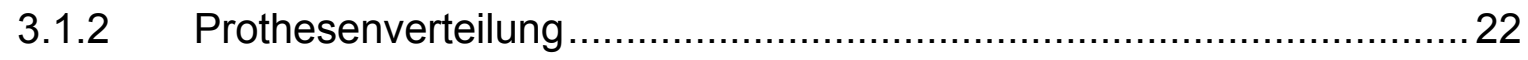

3.1.3 Topographie und Anzahl der Pfeilerzähne ……...............................2 23

3.1.4 Vitalität und Lockerungsgrad der Pfeilerzähne .................................2 24 
3.1.5 Antagonistische Versorgung.....

3.2 Ereigniszeitanalysen der Resilienzteleskopprothesen nach

Kaplan-Meier. 26

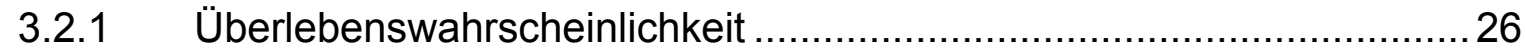

3.2.2 Erfolgswahrscheinlichkeit (keine Komplikation/Intervention) ...............27

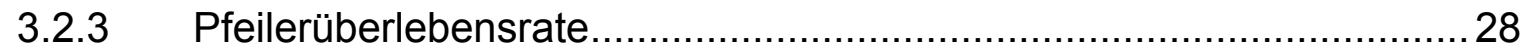

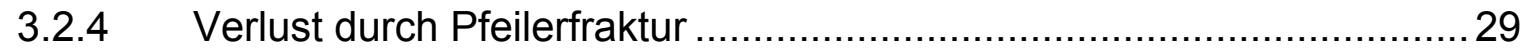

3.2.5 Biologische Erfolgsrate ............................................................. 30

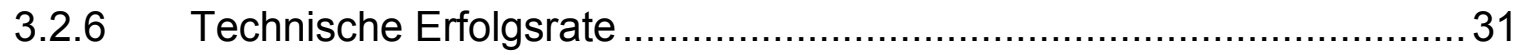

3.3 Ereigniszeitanalysen in Abhängigkeit von möglichen Risikofaktoren ... 32

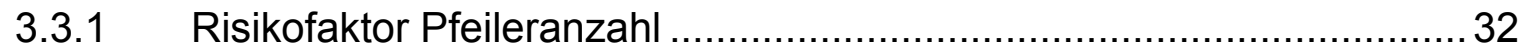

3.3.1.1 Überlebenswahrscheinlichkeit in Abhängigkeit von der Pfeileranzahl.. 32

3.3.1.2 Erfolgswahrscheinlichkeit in Abhängigkeit von der Pfeileranzahl ......... 34

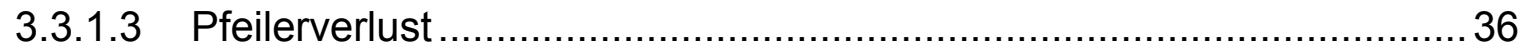

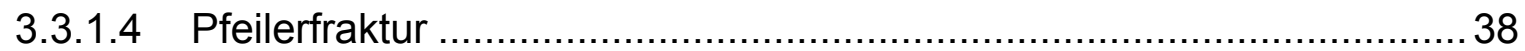

3.3.1.5 Erfolgswahrscheinlichkeit bezogen auf Retentionsverlust.................. 40

3.3.2 Risikofaktor Antagonistische Versorgung ....................................... 42

3.3.2.1 Überlebenswahrscheinlichkeit in Abhängigkeit von der antagonistischen Versorgung

3.3.2.2 Erfolgswahrscheinlichkeit in Abhängigkeit von der antagonistischen

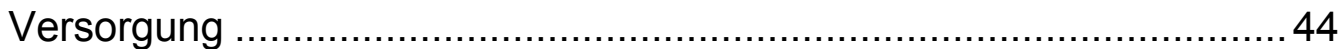

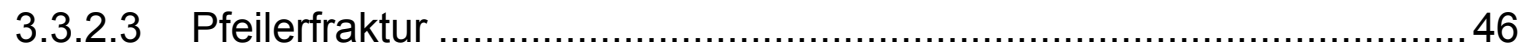

3.3.2.4 Erfolgswahrscheinlichkeit bezogen auf Prothesenbasisfraktur............ 48

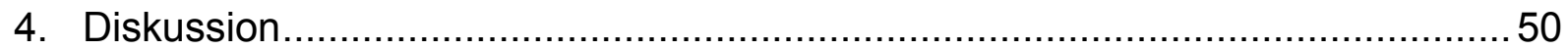

4.1 Diskussion der Ergebnisse ........................................................ 50

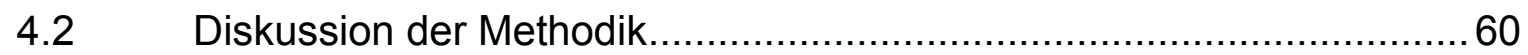

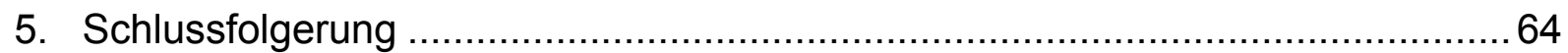

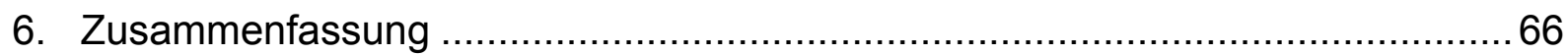

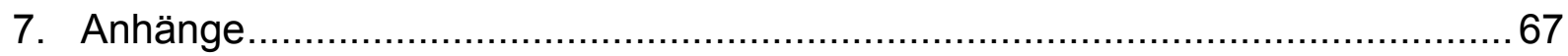

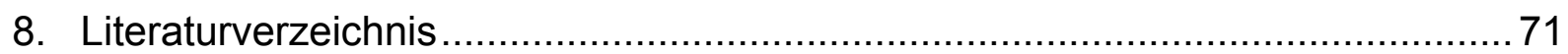




\section{Abkürzungsverzeichnis}

BEMA

et al.

insg.

Jg.

Koef

Kombi-Zahnersatz

$\mathrm{mm}$

NA

$\mathrm{N}$

OK

RTP

u. a.

UK
Bewertungsmaßstab zahnärztlicher Leistungen

et alii/ et aliae/ et alia, bedeutet "und andere"

insgesamt

Jahrgang

Koeffizient

kombiniert festsitzend-abnehmbarer Zahnersatz

Millimeter

Not Available, nicht verfügbar

Newton

Oberkiefer

Resilienzteleskopprothese

unter anderem

Unterkiefer 


\section{$1 \quad$ Einleitung}

Die Erhaltung oder Wiederherstellung der Funktion des Kauorgans sollte Ziel jeglicher zahnärztlicher Therapie sein (Jüde et al. 1979).

Der Anstieg der Lebenserwartung, eine gute Versorgung mittels Zahnersatzes und die wachsende Bedeutung der Prophylaxe hat zu einem längeren Erhalt des Restgebisses geführt. Der Bedarf und die Notwendigkeit der Versorgung von älteren Menschen mit Totalprothesen nimmt immer mehr ab oder entsteht erst viel später als bisher. Die verbleibenden Restzähne müssen unter funktionellen und ästhetischen Gesichtspunkten so gut prothetisch versorgt werden, dass dieser Zahnersatz den steigenden Ansprüchen der Patienten gerecht wird. Der damit verbundene Begriff „Lebensqualität“ gewinnt in der Zahnmedizin zunehmend an Bedeutung. Kothe et al. (2003) konnten in ihrer Untersuchung zeigen, dass sich signifikante Verbesserungen nach neuer teleskopierender Versorgung im Hinblick auf „Äußere Erscheinung“, „Komfort“ und „Eingeschränkte Nahrungsaufnahme“ ergaben.

In Abhängigkeit von der Anzahl, Wertigkeit und Verteilung der noch vorhandenen Zähne sowie der Compliance und finanziellen Möglichkeit des Patienten erfolgt eine Versorgung mittels einer festsitzenden, abnehmbaren oder kombiniert festsitzendabnehmbaren Konstruktion. Ziel dabei sollte es sein, den Zustand des Restgebisses zu erhalten und einer Verschlechterung entgegenzuwirken. Der Zahnersatz soll in keinem Fall einen negativen Einfluss auf das stomatognathe System haben. Bereits Studien aus dem Jahr 1977 belegen, dass der Eingliederungszustand durch regelmäßige Kontrollen - nach Abschluss der Behandlung - über Jahre gehalten werden kann (Bergmann et al. 1977).

Die abnehmbare Zahnprothese wird über spezielle Verbindungselemente am Restgebiss befestigt. Diese erfüllen sowohl die Aufgabe einer Kippmeider- und Haltefunktion gegen auf die Prothese einwirkende Abzugskräfte als auch eine Stützund Schubverteilungsfunktion, um auftreffende Kaukräfte auf die Pfeilerzähne weiterzuleiten oder umzuverteilen (Stark 1996).

Spezielle Ankerelemente als Verbindung zwischen Teilprothese und Pfeilerzahn lassen sich in Stege, Geschiebe, Klammern und Doppelkronen differenzieren (Freesmeyer 1987). 


\section{$1.1 \quad$ Das reduzierte Restgebiss}

\subsubsection{Definition}

Sind bei einem Patienten in einem Kiefer nicht mehr als drei oder vier (gegebenenfalls parodontal geschwächte) Zähne vorhanden, liegt ein stark reduziertes Restgebiss vor (Jung 1989).

\subsubsection{Erscheinungsbild}

In vielen Fällen sind die noch vorhanden Restzähne durch kariöse, parodontale oder abrasive Schäden sowie durch eine fehlende okklusale Abstützung gekennzeichnet (Jung 1989). Des Weiteren ist die Verteilung der Zähne im Kiefer häufig statisch ungünstig. Meist fehlt ein Unterstützungspolygon, und die Stützlinie verläuft quer durch die Prothesenbasis (Körber 1980, von Majewsky 1989).

Eine insuffiziente prothetische Versorgung und die zunehmende Atrophie des Kieferknochens führen dazu, dass sich das Verhältnis der Hebelarme am Pfeilerzahn ändert. Dabei wird der koronare Hebelarm länger als der radikuläre Widerstandsarm und eine Belastbarkeit des Zahnes in horizontaler Richtung kritischer. Außerdem wird dieser Verlauf häufig von einer Taschenbildung, Schwund des Alveolarknochens und einer Entzündung der marginalen Gingiva unterstützt. Das Resultat ist eine erhöhte Zahnlockerung (Dolder und Wirz 1982).

In vielen Fällen prägen verschieden lange, zahnlose, prognostisch ungünstige, gekrümmt verlaufende, „akut oder prospektiv schlechte“ flache Kieferkammabschnitte das Erscheinungsbild (Ludwig 1983; Sauer 1986; Jung 1989).

\subsubsection{Die Notwendigkeit für den Erhalt von Restzähnen}

Aus zahnärztlich-prothetischer Sicht ist der Erhalt der Zähne im Restgebiss äußerst sinnvoll und wichtig. Dabei spielt die funktionelle Belastung der Wurzeln, welche dem Abbau des Alveolarfortsatzes entgegenwirken, eine wichtige Rolle (Jung 1989). 
Da dieser für einen langfristigen Behandlungserfolg sehr wichtig ist, sollte der Zahnersatz selbst keinen oder nur einen geringen Einfluss auf die Prothesenlagergewebe nehmen (Ludwig 1983).

Des Weiteren sind die Restzähne zur Verankerung und dem damit verbundenen Halt des Zahnersatzes sehr wertvoll. So kann es durchaus sinnvoll sein, Zähne vorübergehend $\mathrm{zu}$ belassen, auch wenn sie langfristig entfernt werden müssen (Nitschke und Müller 2002). Vor allem im Unterkiefer ist dies aufgrund der ungünstigen Morphologie sinnvoll. Außerdem können die Restzähne, zum Beispiel bei fehlender Kieferkamm-Retention im Oberkiefer (Tuber maxillae), eine Verbesserung des Halts herbeiführen.

Sowohl die propriozeptive Steuerung der Kaumuskulatur durch die Erhaltung der Rezeptoren im Parodontium als auch die Sprachlautbildung können so erhalten werden (Kobes 1969).

Dem Erhalt der Restzähne kommt ebenfalls auch ein psychologischer Wert zu. Die Adaptation an den Zahnersatz erfolgt leichter, selbst wenn nur noch eine Restwurzel als Anker dient (Kobes 1969).

Es ist wichtig, bei der Planung eine mangelnde oder gar fehlende Adaptationsfähigkeit des älteren Patienten an einen neuen Zahnersatz abschätzen und berücksichtigen zu können (Bär et al. 2009).

\subsection{Doppelkronen}

\subsubsection{Geschichte}

Doppelkronen werden seit über 100 Jahren zur Verankerung von herausnehmbarem Zahnersatz verwendet (Lehmann und Gente 1988). R. W. Starr, ein in Philadelphia/USA praktizierender Arzt, beschrieb im Jahre 1886 erstmalig abnehmbare Brücken, welche über Doppelkronen abgestützt waren. Dabei bestand die Primärkrone aus zylindrischen Ringbandkappen (Lehmann und Gente 1988). 1929 wurde die teleskopierende Versorgung von Häupl nach Europa gebracht (Häupl und Reichborn-Kjennerud 1929). 
Seine Schüler Rehm und Böttger haben in einer Vielzahl von klinischen Fällen telekopierende Verbindungselemente und Teleskopkronen zur Verankerung von Zahnersatz beschrieben (Rehm 1940, 1952; Böttger 1953, 1961; Böttger et al. 1965), so dass sie ab 1950 vermehrt zur Verankerung abnehmbarer Teilprothesen verwendet wurden. Hofmann und Graber beschrieben 1966 die resiliente Lagerung von Teilprothesen durch Doppelkronen mit Spielpassung für das stark reduzierte Restgebiss (Hofmann 1966; Graber 1966).

1968 führte K. H. Körber das System der konischen Doppelkronen ein, welche eine Retention durch Verkeilung erreichen. Der Einsatz zusätzlicher Halteelemente wurde 1971 von Lehmann beschrieben (Wenz und Kern 2007).

Somit wurde der Weg in die zahnärztliche Praxis geebnet.

\subsubsection{Definition der Doppelkronen}

In der Zahnmedizin setzt sich eine Doppelkrone aus einer fest auf den Pfeiler aufzementierten Primärkrone und einer mit dem abnehmbaren Zahnersatz verbundenen Sekundärkrone zusammen (Lehmann und Gente 1988). Sie sind als Verankerungselemente für herausnehmbaren Zahnersatz gut geeignet (Freesmeyer 1987).

\subsubsection{Klassifikation und Auswahlschema zur Therapie}

Die Auswahl der Doppelkrone als Verankerungselement erfolgt nach einem üblichen Schema. Abbildung 1 zeigt einen Überblick über den standardisierten Ablauf.

Ist der vorhandene Zahnbestand belastbar, sollte der Patient mit einem parodontal abgestützten Zahnersatz prothetisch versorgt werden. Dazu zählen Konuskronen, Teleskopkronen sowie Doppelkronen mit einem zusätzlichen Halteelement.

Auf schleimhautgetragene Prothesen sollte bei einem stark reduzierten Lückengebiss zurückgegriffen werden. 
Dabei hat das Verankerungselement keine abstützende Funktion und ist somit ein Element der beweglichen Lagerung (Lehmann und Gente 1988).

Der Zahnersatz kann sich unter Belastung in die Kieferschleimhaut entsprechend der Resilienz am Ort der Verankerung einlagern (Lehmann und Gente 1988). Es handelt sich hierbei um so genannte Resilienzteleskopprothesen.

Die Verwendung der Doppelkrone als Element der starren oder beweglichen Lagerung wird erst nach ausführlicher Befundung und Erstellung eines Behandlungsplanes entschieden und umgesetzt.

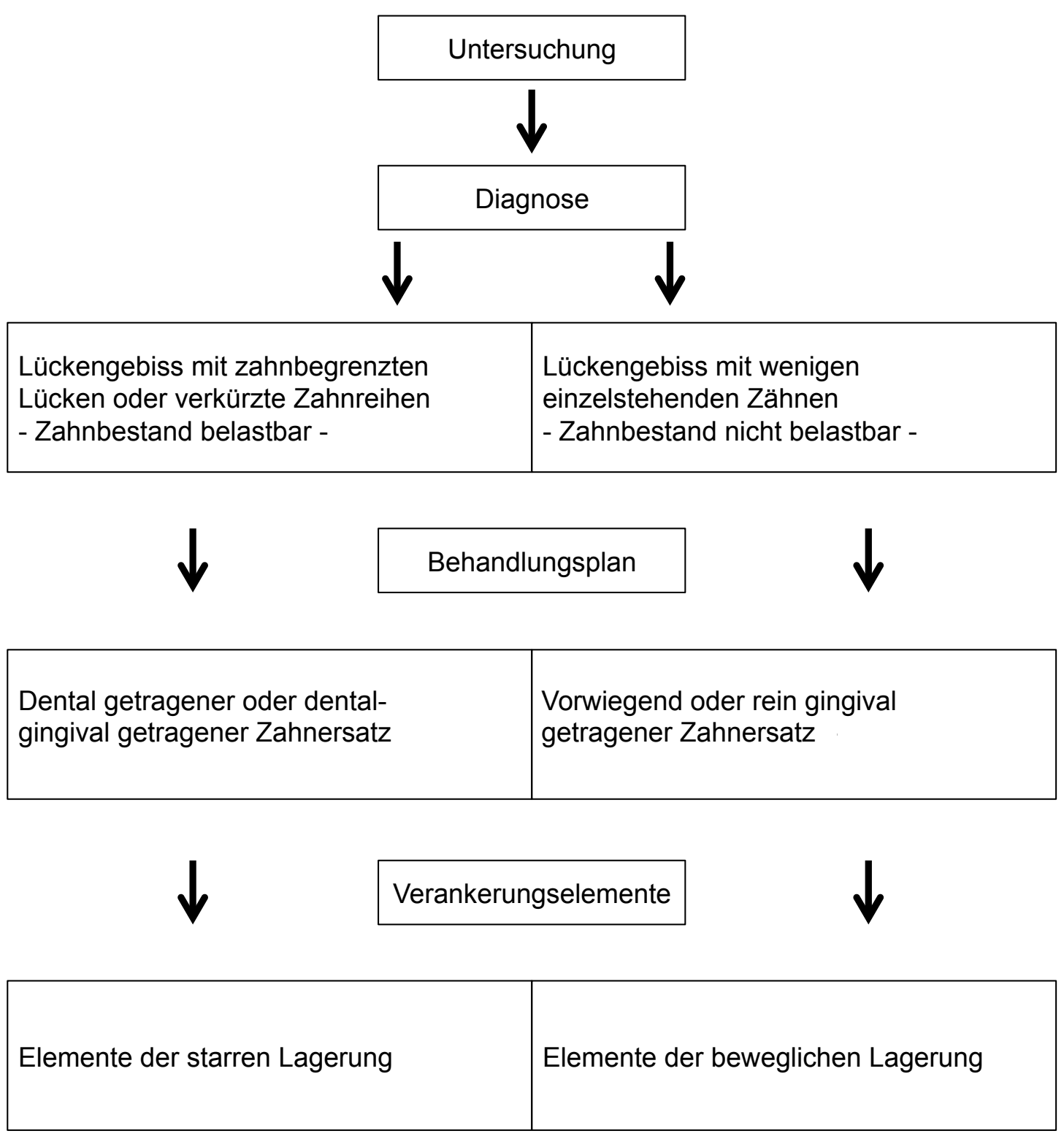

Abb. 1: Auswahlschema zur Therapie des Restgebisses, modifiziert nach Lehmann und Gente 1988, S. 107 
In der Doppelkronentechnik differenziert man zwischen Teleskopen, Konuskronen und Doppelkronen mit zusätzlichem Halteelement, die der starren Lagerung angehören sowie Resilienzteleskopen, als Elemente der beweglichen Lagerung (Lehmann und Gente 1988; Wenz et al. 1999).

\subsubsection{Doppelkronen als Elemente der starren Lagerung}

Doppelkronen, welche Elemente der starren Lagerung besitzen, können eine Halteund Stützfunktion ausüben (Lehmann und Gente 1988). Dabei lässt sich die Stützfunktion auf die exakte Passung der Sekundärkrone auf der Primärkrone zurückführen. Die Haltewirkung wird, abhängig vom verwendeten Doppelkronensystem, auf unterschiedlichem Weg erreicht (Lehmann und Gente 1988).

Abbildung 2 zeigt eine schematische Darstellung der verschiedenen Doppelkronensysteme.
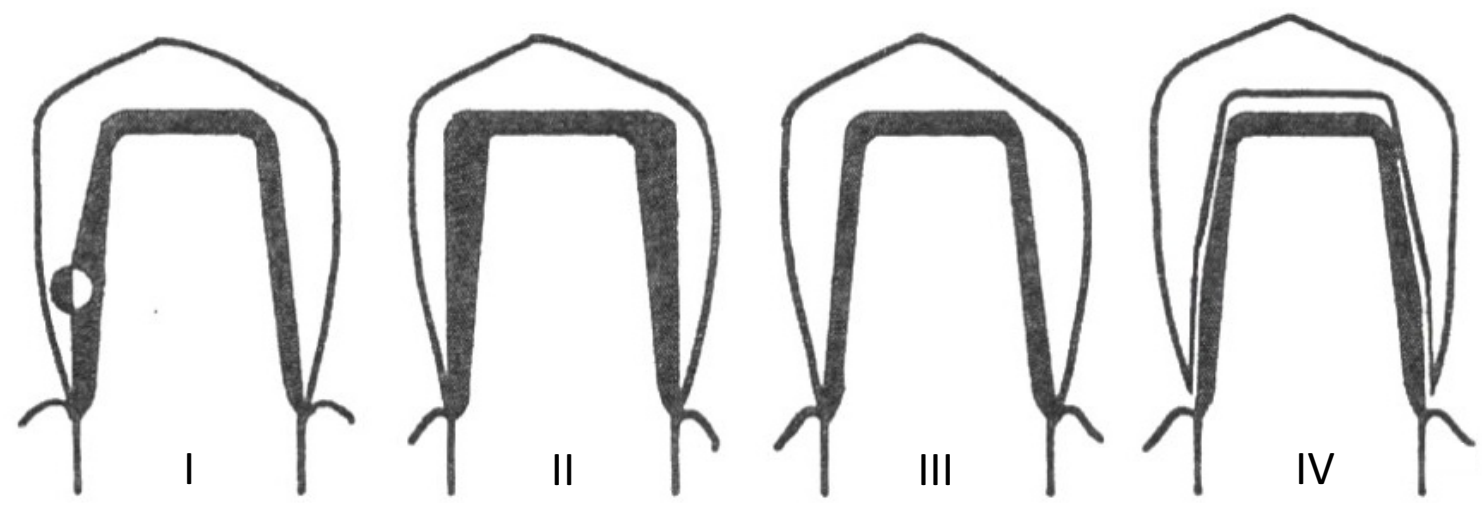

Abb. 2: Schematische Darstellung der verschiedenen Doppelkronentypen, I-IV Doppelkronen als Elemente der starren Lagerung, I = Doppelkrone mit Halteelement (Riegel), II = Teleskopkrone, III = Konuskrone, IV = Doppelkrone als Element der beweglichen Lagerung (mit Resilienzspielraum zwischen Patrize und Matrize), modifiziert nach Lehmann und Gente 1988, S. 108 


\subsubsection{Teleskopkronen}

Eine in Deutschland sehr verbreitete Möglichkeit zur prothetischen Versorgung stark reduzierter Restgebisse stellen Teleskopprothesen dar (Diedrichs 1990; Stark 1996). Dabei entsteht die Haltewirkung durch die Friktion parallelisierter Flächen (Böttger und Gründler 1970; Böttger 1973). Die Stützfunktion entsteht aus dem Formschluss zwischen Außen- und Innenkrone (Stark 1996). Somit verfügen die teleskopierenden Prothesenanker über eine gute Halte- und Stützfunktion. Im Frontzahngebiet muss die Primärkrone über mindestens zwei gegenüberliegende, streng parallelisierte Wände verfügen, im Seitenzahngebiet sind zirkulär parallele Wände möglich (Lehmann und Gente 1988).

Während des Fügens oder Trennens entstehen zwischen den Oberflächen der Primär- und Sekundärfläche sogenannte Gleit- und Haftreibungskräfte, die in der zahnärztlichen Literatur auch als Friktionskräfte bezeichnet werden (Böttger 1961; Lehmann und Gente 1988; Meyer 1983; Reither 1978; Stüttgen 1985). Diese sind auf die zwischenmolekularen Wechselwirkungen zwischen den Oberflächenschichten beider Kronenanteile, den sogenannten Kaltschweißbrücken, und den folgenden Adhäsionskräften sowie dem mechanischen Kontakt, das heißt dem Vielpunktkontakt zwischen Primär- und Sekundärkrone begründet (Becker 1983).

Es werden geschlossene (Vollteleskope) von offenen Formen (Teilteleskope) unterschieden (Lehmann und Gente 1988). Es handelt sich um einen kombiniert festsitzend-abnehmbaren Zahnersatz.

Die Pfeilerzähne werden über die herausnehmbare Prothesenkonstruktion indirekt miteinander starr verbunden und somit sekundär geschient, wodurch eine funktionell günstige Belastung zustande kommt (Böttger 1953; Häupl 1959; Häupl und Reichborn-Kjennerud 1929; Rehm et al. 1962). Der außergewöhnlich feste Sitz und Halt der Prothese kann des Weiteren als vorteilhaft angesehen werden (Stark 1996). Dies kommt durch die sekundäre Verblockung der einzelnen Pfeilerzähne über die Suprakonstruktion zustande. Das gute Kau- und Sprachvermögen, die kurze Eingewöhnungszeit sowie die gute Ästhetik wurden von den Patienten als sehr positiv bewertet (Adam 1984; Gernet et al. 1983; Kothe et al. 2003). 
Im Vergleich zu Klammer-Modellgussprothesen, bei welchen es vor allem im Frontzahnbereich zur Sichtbarkeit der Klammerarme kommt, ist bei der Teleskoptechnik eine bessere ästhetische Gestaltung gegeben (Teubner et al. 2007). Des Weiteren stellte man in Studien von Bergmann (1987), Stark (1993) und Ettinger und Jakobson (1997) fest, dass die Pflege und die damit verbundene Reinigung, im Vergleich zu anderen Versorgungen, keine größeren Anforderungen an die Patienten stellt. Eine regelmäßige Nachsorge sowie eine gute Motivation und Instruktion der Patienten müssen dabei jedoch ein wichtiger Grundbaustein der prothetischen Behandlung sein.

Langer gab in seiner Studie an, dass Teleskope, bedingt durch die axiale Belastung und die gute körperliche Fassung der Pfeilerzähne, zu einer Festigung der gelockerten Zähne führen können (Langer 1981). Sowohl aus wirtschaftlicher als auch aus finanzieller Sicht ist die leichte Erweiterbarkeit der Teleskoparbeit als sehr vorteilhaft anzusehen (Stark und Schrenker 1998; Vosbeck 1989; Wenz et al. 2001). Indiziert ist die Teleskopprothese auch bei einem stark reduzierten Restgebiss (Jacoby und Gasser 1973; Schwanwede und Anderseck 1985), und/oder bei statisch ungünstiger Pfeilerverteilung (Wupper 1986) und unsicherer Prognose einzelner Zähne im parodontal geschädigten Gebiss (Krämer und Weber 1990; Vosbeck 1989). Es bedarf eines hohen finanziellen und technischen Aufwandes zur Herstellung einer qualitativ hochwertigen Teleskoparbeit. Das Sekundärteleskop wird, im Gegensatz zu den Konuskronen, mit Kunststoff verblendet.

Nicht zu vernachlässigen ist der eintretende Materialverschleiß infolge einer dauernden Bewegungsreibung an allen Flächen beim Ein- und Ausgliedern (Körber 1974).

Die Anzahl, Parallelität sowie Oberflächenbeschaffenheit der Teleskope, der Parodontalzustand der Restzähne und die Geschicklichkeit des Patienten bestimmen die Einstellung der Friktion (Kimmel 2007; Koeck 1996). Die jeweils verwendete Legierung hat dabei einen geringeren Einfluss auf die Friktion (Kimmel 2007). Es ist darauf hinzuweisen, dass der Name Teleskopkrone gerne synonym für den Begriff Doppelkrone verwendet wird. Dies entspricht jedoch nicht den Tatsachen, da es sich bei der Teleskopversorgung um eine parallelwandige Konstruktion handelt. 


\subsubsection{Konuskronen}

Die von K. H. Körber entwickelte Konuskrone beruht auf einer Keilwirkung zwischen Primär- und Sekundärkrone (Körber 1974). Diese entsteht durch die konische Gestaltung des Innenteleskopes. Abhängig vom Konuswinkel zwischen Patrize und Matrize resultiert die Haltewirkung (Lehmann und Gente 1988). Dieser Konuswinkel ist definiert als Neigung der Außenfläche der Primärkrone zur Einschubrichtung (Lehmann und Gente 1988).

Die Haftkraft ist umso kleiner, je größer der Konuswinkel gewählt wird. Sie beträgt 8 bis $10 \mathrm{~N}$ bei einem Winkel von $6^{\circ}$ bis $8^{\circ}$ (Lehmann und Gente 1988).

Für ein gesundes Parodont sind Extrusionskräfte dieses Ausmaßes unschädlich. Allerdings sollten sie nicht höher gewählt werden, um Traumatisierungen des Zahnhalteapparates zu vermeiden (Lehmann und Gente 1988).

Es ist möglich, parodontal schwächere Zähne, sogenannte „Stützkoni“, durch eine Vergrößerung des Konuswinkels und damit resultierender Haftverminderung mit einzubeziehen (Lehmann und Gente 1988). Bei Konuskronen ist eine Haftreibung bei Bewegung nicht möglich und somit ein Materialverschleiß - im Gegensatz zur Teleskopversorgung - ausgeschlossen (Körber 1974). Erst bei endgültiger Lage treten die Berührungsflächen zueinander in Haftung. Aus diesem Grund kann die Versorgung nach dem ersten Lösedruck reibungslos abgenommen werden (Körber 1974).

\subsubsection{Doppelkronen mit Halteelement}

Hierbei wird der Halt zwischen Innen- und Außenteleskop durch ein zusätzliches Halteelement bestimmt. Nach Möglichkeit wird die Primärkrone parallelisiert und die Sekundärkrone sitzt passgenau, jedoch ohne Friktion, mit Spielpassung auf dem Innenteleskop (Lehmann und Gente 1988). 
Es wurde in einigen Studien nachgewiesen, dass mit zunehmender Tragedauer die Friktion von Teleskopen nachlässt (Freesmeyer 1987; Meyer 1983; Vosbeck 1989). Somit versuchte man diesem Problem entgegenzuwirken. Dabei gehört der Riegel zu den klassischen Halteelementen (Lehmann und Gente 1988). Es folgten weitere Retentionssysteme, wie das TK-Snap-System 1991 (Firma Si-Tec), Friktionsstifte (Weber) sowie Titannitridbeschichtungen (Gütschow) 1993.

Im Gegensatz zur oben genannten Studie stellte Hagener 2006 fest, dass er in seinen Versuchen zum Abnutzungsverhalten bei Teleskopen keinen Friktionsverlust feststellen konnte. Teilweise stieg die Haftkraft sogar an, und die Pfeilerzähne wurden überlastet.

\subsubsection{Doppelkronen als Elemente der beweglichen Lagerung bei Resilienzteleskopprothesen}

Dieses Doppelkronensystem, welches weder eine Halte- noch eine Stützfunktion besitzt, ist im stark reduzierten Restgebiss mit parodontal geschwächten Zähnen indiziert (Lehmann und Gente 1988). Die Resilienzteleskopprothese folgt diesem Funktionsprinzip, das im Folgenden beschrieben wird.

Hofmann beschrieb 1966 erstmals diese Doppelkronenart (Hofmann 1966). Sie ist sowohl unter dem Namen "Teleskopierende Totalprothese" (Hofmann und Ludwig 1973), „Cover-Denture-Prothese“ (Hofmann 1966) als auch Resilienzteleskopprothese bekannt.

\subsubsection{Funktionsprinzip}

Das Funktionsprinzip dieses Doppelkronensystems entspricht einer rein gingivalgetragenen Totalprothese. Dabei werden okklusal einwirkende Kräfte vor allem von dem Knochen und der Schleimhaut aufgenommen, eine Belastung der Pfeilerzähne findet primär nicht statt.

Die überkronten Restzähne sichern die Prothese gegen kippende Bewegungen, übernehmen eine gewisse Führung und werden erst nach Erschöpfung der Schleimhautresilienz belastet (Hofmann und Ludwig 1973). 
Hofmann und Ludwig beschrieben 1973 diesen Vorgang als ein ständiges Wechselspiel zwischen Belastung und Entlastung des Zahnes sowie des Schleimhautteguments. Der Halt dieses Doppelkronensystems wird über die funktionelle Randgestaltung (Ventilrand) wie bei der Totalprothese erreicht (Lehmann und Gente 1988).

In Ruhelage besteht okklusal zwischen Innen- und Außenteleskop ein Abstand, der sog. Resilienzspielraum, welcher der Schleimhautresilienz entspricht (Lehmann und Gente 1988). Je nach Beschaffenheit liegt diese zwischen 0,2 und 0,7 mm (Körber 1954; Rehm et al. 1962). In der Regel wird eine vertikale Spielpassung von 0,3 mm, bei einem straffen Tegument von 0,2 mm eingearbeitet (Hofmann und Ludwig 1973). Der Resilienzspalt sollte jedoch nie größer als $0,5 \mathrm{~mm}$ sein (Hofmann und Ludwig 1973). Im unteren Drittel ist eine Parallelisierung der Seitenwände zu erkennen (Abb. 3) (Lehmann und Gente 1988). Innen- und Außenteleskop müssen sowohl bei Kaubelastung als auch bei Entlastung ohne Friktion aneinander vorbei gleiten können, denn nur dann wird der Zahnersatz wieder in die eigentliche Ausgangslage gebracht (Lehmann und Gente 1973).

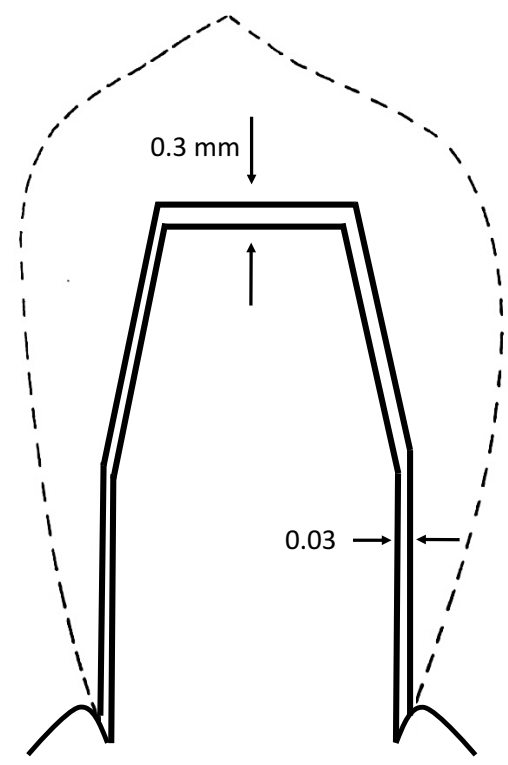

Abb. 3: Prinzip der Doppelkrone mit Resilienzspielraum, modifiziert nach Hofmann 1976, S. 40 
Sowohl edelmetallhaltige als auch edelmetallfreie Legierungen können zur Herstellung dieser Doppelkronen verwendet werden, da bei der Modellation die Retentionen berücksichtigt werden (von Majewsky 1989).

Lehmann und Gente wiesen 1988 mit einigen Untersuchungen darauf hin, dass es aus parodontalhygienischer Sicht erforderlich ist, alle überdeckten Bereiche des marginalen Parodonts hohlzulegen und den extraalveolären Teil der Pfeilerzähne nicht länger zu halten als den Intraalveolären. Aus heutiger Sicht ist die Indikation von teleskopierenden Totalprothesen umstritten. Sowohl von Majewsky (1989) als auch Strub et al. (2011) beschrieben, dass der eingearbeitete Resilienzspielraum aufgrund der Einlagerung der Prothese mittelfristig verloren geht. Aus diesem Grund sind regelmäßig durchgeführte Kontrollen von großer Bedeutung, um einem Absinken des Prothesensattels durch eine Unterfütterung entgegenzuwirken (von Majewsky 1989). Auch eine parodontale Problematik an den Pfeilerzähnen stellt eine große Schwierigkeit dar.

\subsubsection{Indikation}

Die Resilienzteleskopprothese ist bei Patienten mit maximal 3 Restzähnen pro Kiefer indiziert. Alternativ könnte der Einsatz einer Totalprothese diskutiert werden (Hofmann und Ludwig 1973).

Die Restzähne sollen den Funktionswert der Prothese bezüglich Halt und Stabilität verbessern sowie den späteren Übergang zu einer zahnlosen Versorgung erleichtern (Hofmann und Ludwig 1973).

\subsubsection{Bewährung von Resilienztelekopprothesen im stark reduzierten Restgebiss}

Hofmann und Ludwig konnten 1973 bei 78 Prothesen mit 163 Resilienzteleskopen feststellen, dass der Lockerungsgrad der Restzähne nach Eingliederung der Prothese innerhalb der ersten 3 Jahre abnahm. Diese Tendenz war ebenfalls an der Taschentiefe zu erkennen. Mit zunehmender Tragedauer kam es jedoch zu einer Erhöhung der Mobilität und der Sondierungstiefe. 
Es wurden keine Angaben über das Auftreten von Sekundärkaries, Prothesenreparaturen und Nachbehandlungen an den Pfeilern gemacht. Abschließend konnte man feststellen, dass die Zielsetzung, die Funktion der Totalprothese zu erhöhen, erfüllt wurde (Hofmann und Ludwig 1973).

Pöggeler untersuchte 1995106 Prothesen auf insgesamt 236 Resilienzteleskoppfeilern nach 2 bis 11 Jahren. Dabei waren während der Beobachtungsdauer $14 \%$ der Zähne verloren gegangen, wobei die Überlebenswahrscheinlichkeit der Pfeilerzähne im Unterkiefer mit 92\% nach 5 Jahren besser als im Oberkiefer mit 86\% war. Häufigste Indikation zur Extraktion war eine Parodontitis marginalis profunda. Im Unterschied zu anderen Studien entwickelte sich zwar die Lockerung günstiger, die Taschentiefen nahmen jedoch zu. Pöggeler beschrieb weiterhin ein erhöhtes Auftreten von Rissen bei Unterkieferprothesen (UK 32,8\%, OK 23,4\%) sowie einen schlechten Halt und erhöhten Unterfütterungsbedarf (UK 25\%, OK 11\%) (Pöggeler 1995).

Wenz et al. werteten 1999 in ihrer retrospektiven Studie 111 doppelkronenverankerte Teilprothesen aus und untersuchten dabei das Risiko des Pfeilerverlustes bei 392 Zähnen. Bei starrer Prothesenlagerung ( $\geq 4$ Pfeilerzähne) lag das Risiko nach 5 Jahren bei $4,8 \%$ und bei $8,9 \%$ nach 10 Jahren. Die resiliente Lagerung ( $\leq 3$ Pfeilerzähne) zeigte mit 8,7\% nach 5 bzw. 13,5\% nach 10 Jahren ein höheres Pfeilerverlustrisiko (Wenz et al. 1999). Es konnte anhand der Ergebnisse gezeigt werden, dass Doppelkronen mit Spielpassung gleichermaßen gut zur Verankerung von starr als auch resilient gelagerten Teilprothesen geeignet sind (Wenz et al. 1999). Hervorgehoben wurde im Zusammenhang mit Kieferdefekten, dass eine strenge Pfeilerselektion für den Langzeiterfolg von größerer Bedeutung ist als eine ungünstige Belastungssituation (Wenz et al. 1999).

Coca und Kollegen stellten 2000 in ihrer klinischen Langzeitstudie für Resilienzteleskoppfeiler einen Verlust im Oberkiefer nach durchschnittlich 3,4 Jahren, im Unterkiefer nach 4,5 Jahren (Kaplan-Meier-Analyse) fest. Dabei waren Oberkieferzähne mit einer Wahrscheinlichkeit von 68\% nach 7 Jahren extrahiert worden, untere Pfeilerzähne mit einer Wahrscheinlichkeit von 73\% nach 8 Jahren (Coca et al. 2000). 
Wenz und Kollegen untersuchten 2001 in ihrer Langzeitbewährungsstudie 126 Prothesen mit Spielpassung. Dabei berichteten sie von einer Überlebensrate der Pfeilerzähne von $84 \%$ nach 5 Jahren und $66 \%$ nach 10 Jahren. Es zeigte sich kein signifikanter Unterschied zwischen resilienter ( $\leq 3$ Pfeilerzähne) und starrer Lagerung ( $\geq 4$ Pfeilerzähne) (Wenz et al. 2001).

Coca und Klimek verglichen 2002 im Unterkiefer magnetgehaltene Totalprothesen, Cover-Dentures mit Spielpassungsteleskopen und klammerverankerte Modellgussprothesen nach einer durchschnittlichen Tragedauer von 6,5 Jahren miteinander. Dabei konnte festgestellt werden, dass ein Zahnverlust bei 12\% der Primärkronen zu verzeichnen war und die Überlebenswahrscheinlichkeit bei Pfeilerzähnen mit Primärkronen bei $73 \%$ lag. Magnetwurzelkappen (79\%) und Modellgussprothesen (82\%) wiesen bessere Überlebenswahrscheinlichkeiten auf, was bei Magnetpfeilern auf das günstigere Kronen-Wurzel-Hebelverhältnis und die höhere Pfeileranzahl bei Modellgussprothesen zurückzuführen war (Coca und Klimek 2002).

Schwindling und Kollegen stellten 2014 in ihrer Studie eine Überlebenswahrscheinlichkeit von 90,4\% nach 5 Jahren fest, in welche 34 Resilienzteleskopprothesen einbezogen wurden. Außerdem kamen sie zu dem signifikanten Ergebnis, dass schwerwiegende Komplikationen bei Einbeziehung von Resilienzteleskopprothesen, im Vergleich zu Teleskop- oder Konuskronen, 2,2-mal vermehrt auftraten. Zudem wurde ebenfalls ein negativer Effekt einer geringen Pfeileranzahl hinsichtlich der Überlebenszeit der Versorgungen beschrieben (Schwindling et al. 2014). 


\subsection{Zielsetzung und Problemstellung}

In der Poliklinik für Zahnärztliche Prothetik der Medizinischen Fakultät der Universität Göttingen wurden bis zum Jahre 2014 Patienten mit stark reduziertem Restgebiss mit maximal 3 oder weniger Zähnen pro Kiefer - mit Resilienzteleskopprothesen versorgt.

In einer retrospektiven Verlaufsbeobachtung wurde nun die Bewährung von Resilienztelekopprothesen (mit einem jeweiligen Mindestbeobachtungszeitraum von 2 Jahren) untersucht, wobei auch ein frühzeitigerer Verlust dokumentiert wurde. Herangezogen wurden für diese Untersuchung Doppelkronenversorgungen, welche zwischen 2000 bis 2012 angefertigt wurden.

Diese aktenbasierte Beobachtungsstudie zielte auf eine Einschätzung der Resilienzteleskopprothese hinsichtlich Langzeitbewährung, Komplikationen und Nachsorge ab. Es sollte dargestellt werden, ob eine prothetische Versorgung mit diesem Doppelkronensystem aus heutiger, zahnärztlicher Sicht noch sinnvoll erscheint oder ob man sich besser anderer prothetischer Versorgungsmöglichkeiten bedienen sollte, wie zum Beispiel einer Cover-Denture-Prothese ohne Resilienzspielraum.

Folgende Parameter sollten in dieser Studie analysiert werden:

- Welche Überlebensrate der Resilienzteleskopprothesen und der Pfeilerzähne bestand bei dieser Versorgung im Durchschnitt (nach mindestens 2 Jahren Beobachtungszeit)?

- Wie hoch war die Erfolgswahrscheinlichkeit für ein komplikationsfreies Überleben?

- Welchen Einfluss hatte sowohl die Anzahl der Pfeilerzähne als auch die antagonistische Versorgung auf Überlebens-, Erfolgs- und Pfeilerverlustrate? 


\section{$2 \quad$ Material und Methode}

\section{$2.1 \quad$ Studiendesign}

Die vorliegende retrospektive Studie evaluiert die technischen und biologischen Komplikationsraten sowie die Langzeitbewährung von Resilienzteleskopprothesen im Ober- und Unterkiefer, welche in der Regel im Studentenkurs unter Betreuung oder direkt von Zahnärzten der Poliklinik für Zahnärztliche Prothetik der Medizinischen Fakultät der Universität Göttingen in den Jahren 2000 bis 2012 angefertigt und eingegliedert wurden, wobei die Zahntechnik von gewerblichen Labors ausgeführt und die Nachuntersuchungen von Assistenz- und Oberärzten durchgeführt wurden.

\subsection{Auswahl der Patienten}

Die Patientenakten der Poliklinik für Zahnärztliche Prothetik bildeten die Grundlage für diese Beobachtungsstudie. Diese wurden durch ein in der Zahnärztlichen Prothetik immanentes Recall-Karteikartensystem und erstellte Abrechnungspositionslisten, welche bei der Leistung „BEMA 91d“ (Teleskop-/Konuskrone) einen Rückschluss auf eine Teleskopprothese oder Resilienzteleskopprothese gaben, eruiert. Anhand dieser Listen wurden die Akten im Zentralarchiv angefordert und nach vorher festgelegten Auswahlkriterien, nach Prüfung der Dokumentation, ein Patientenkollektiv zusammengestellt. In der vorliegenden Studie wurden ausschließlich Resilienzteleskopprothesen aufgenommen, die der oben genannten Definition entsprachen. Teleskopprothesen mit starrer Lagerung wurden von der Auswertung ausgeschlossen. Alle Patientennamen wurden durch eine fortlaufende Nummerierung einer Zahl zugeteilt, die in einer zweiten Liste dem Vor- und Zunamen zugeordnet werden konnte. Diese Liste unterliegt datenrechtlichem Verschluss (Pseudonymisierung).

Einschlusskriterium war, dass die Patienten von der Poliklinik für Zahnärztliche Prothetik der Medizinischen Fakultät der Universität Göttingen versorgt wurden und eine Beobachtungszeit von mindestens 24 Monaten vorhanden war, wobei Eingliederungs- und letztes Kontrolldatum dokumentiert sein mussten. 
Anhand der Auswahlkriterien konnten somit 221 Patienten mit insgesamt 263 Resilienztelekopprothesen (127 Oberkiefer/136 Unterkiefer) in die Studie einbezogen werden. Diese Prothesenanzahl setzt sich zum einen aus aktuell nachuntersuchten Patienten, das heißt im Jahr 2013, 2014 oder 2015 und zum anderen aus Versorgungen, welche bereits vor oder nach dem festgelegtem Beobachtungszeitraum einen Verlust zu verzeichnen hatten, zusammen.

Es ergab sich eine mittlere klinische Beobachtungsdauer von 64,5 Monaten.

Erfolgte eine prothetische Mehrfachversorgung bei untersuchten Patienten im Laufe der Behandlungszeit, wurde nur die jeweils erste Versorgung ab dem Jahr 2000 pro Kiefer in diese Studie eingeschlossen.

Ein einheitliches Recall-Intervall konnte aufgrund verschiedener Faktoren (zwischenzeitlicher externer Behandler, unregelmäßiges Erscheinen) nicht festgelegt werden und war somit kein Ausschlusskriterium. Es ergab sich eine Geschlechterverteilung von 100 weiblichen und 121 männlichen Patienten.

\subsection{Untersuchte Parameter/Auswertung}

Bei der Aufnahme der Resilienzteleskopprothesen wurde, neben den bereits erwähnten Kriterien, sowohl zum Eingliederungszeitpunkt als auch, wenn vorhanden, bei den Verlaufskontrollen die Vitalität und der Lockerungsgrad des jeweiligen Pfeilers dokumentiert. Des Weiteren wurde die antagonistische Versorgung vermerkt. Komplikationen aller Art wurden zum Zeitpunkt des Auftretens dokumentiert. Sie sind definiert als ein Ereignis, welches eine klinische Intervention erforderte, um den Zahnersatz in Funktion zu halten. Es erfolgte eine Aufteilung in zwei Untergruppen. Dabei handelte es sich um biologische Komplikationen wie das Auftreten einer Sekundärkaries, Füllungen, Vitalitätsverlust, Pfeilerfraktur, parodontale Probleme, Schmerzen, Pfeilerverlust oder Druckstellen und technische Komplikationen wie der Retentionsverlust des Primärteils, Perforationen, Änderungen der Friktion, das zusätzliche Eingliedern einer Schiene, Frakturen eines Prothesenzahnes, der Prothesenbasis oder der Verblendung, Korrekturen der Oklussion, Änderungen der Vertikaldimension, Umstellungen bzw. Austausch der Front oder Seitenzähne und Unterfütterungen. 
Die jeweils erste dokumentierte Komplikation pro Prothese, unabhängig von deren Art, wurde mittels Datum erfasst und anschließend in der Erfolgsanalyse ausgewertet. Dabei ist zu beachten, dass alle biologischen Komplikationen ab dem ersten Tag, nach definitiver Eingliederung der Arbeit, als solche gewertet wurden (außer Druckstellen). Druckstellen und alle Arten technischer Komplikationen wurden erst nach einer Tragezeit von 6 Monaten als solche gewertet, da sie im Zeitraum der ersten 6 Monate als Modifikation noch innerhalb des Herstellungs- bzw. Eingliederungsprozesses eingestuft wurden.

Es wurde sowohl die Anzahl der jeweils aufgetretenen biologischen und technischen Komplikationen als auch die Gesamtzahl errechnet und ausgewertet.

Im Anschluss wurden verschiedene Ereigniszeitanalysen nach Kaplan-Meier erstellt. Zur Evaluation des Einflusses unabhängiger Variablen auf die Dauer der Überlebenszeit der Resilienzteleskopprothesen wurde eine Cox-Regressionsanalyse durchgeführt. Diese multivariate Cox-Regression wurde für jede Variable erstellt.

Die unabhängigen Variablen setzen sich zum einen aus der Pfeileranzahl der Gruppen 'Pfeileranzahl 1' und 'Pfeileranzahl 2', welche der Referenzgruppe 'Pfeileranzahl 3' gegenüber gestellt wurden, zusammen. Zum Anderen wurde die antagonistische Versorgung der Gruppen 'kombiniert festsitzend-abnehmbarer Zahnersatz' und 'Totalprothesen' mit der Referenzgruppe 'festsitzender Zahnersatz' in Zusammenhang gesetzt. Dabei wurden die Ergebnisse zunächst mit Hilfe der Hazard-Funktion interpretiert und anschließend durch den Hazard-Quotient (Hazard Ratio) als Maß für die Stärke eines Zusammenhanges berechnet. „Hazard“ gibt die Wahrscheinlichkeit eines Ereignisses in einer bestimmten Beobachtungszeit an, „Rate" bezieht sich auf die Anzahl der Ereignisse in einer bestimmten Zeit. Die „Ratios" aus beiden Gruppen werden anschließend miteinander verglichen und ins Verhältnis gesetzt (,Ratio“ = Verhältnis).

Wird ein "Hazard Ratio" von 1 ermittelt, kann kein Unterschied zwischen den Gruppen festgestellt werden. Liegt der Wert über 1, ist das Risiko ein Ereignis in einer bestimmten Zeit in der beobachteten Gruppe zu erwarten größer als in der Vergleichsgruppe. Ist der ermittelte Wert kleiner als 1 , ist das Risiko für die beobachtete Gruppe kleiner. 
Es wurden verschiedene Ereignisse vom gleichen Patienten (abhängige Stichprobe) durch eine adjustierte Varianzschätzung berücksichtigt.

Als Ausdruck statistischer Signifikanz wurden p-Werte kleiner als 0,05 festgelegt.

\section{$2.4 \quad$ Art der Dokumentation}

Alle Patientendaten wurden kontinuierlich vom jeweilig behandelnden Zahnarzt dokumentiert. Dabei wurden Nachsorgemaßnahmen sowie Komplikationen schriftlich in der Akte des Patienten festgehalten.

Die aufgenommenen Daten wurden in einer Excel Tabelle erfasst und anschließend ausgewertet. Dabei wurden Zahlen in fortlaufender Reihenfolge des Einschlusses als Verschlüsselung des jeweiligen Vor- bzw. Nachnamens im Sinne einer Pseudonymisierung verwendet. 


\section{$3 \quad$ Ergebnisse}

Die vorliegende Studie identifizierte 221 Patienten, welche in der Poliklinik für Zahnärztliche Prothetik der Medizinischen Fakultät der Universität Göttingen in den Jahren 2000 bis 2012 mit Resilienzteleskopprothesen versorgt wurden.

Die folgende Auswertung und Analyse bezieht sich zum einen auf aktuell nachuntersuchte Resilienzteleskopprothesen und zum anderen auf Versorgungen, die zwischenzeitlich bereits zu Verlust gegangen waren.

Es wurden verschiedene Häufigkeitsverteilungen ermittelt und Ereigniszeitanalysen nach Kaplan-Meier durchgeführt. Des Weiteren wurde der Einfluss von Pfeileranzahl und antagonistischer Versorgung für jede zeitabhängige Variable in einem multivariaten Cox-Regressionsmodell untersucht.

Es ergaben sich individuelle Beobachtungszeiträume von 3 bis 179 Monaten mit einer mittleren klinischen Beobachtungsdauer von 64,5 Monaten (5,4 Jahre) und einer Standardabweichung von $\pm 34,8$ Monaten.

Die Ergebnisse der statistischen Analyse sind im Anhang dargestellt (Tabellen 11 bis 14).

Die statistische Auswertung der ermittelten Daten erfolgte durch die Abteilung Medizinische Statistik der Georg-August-Universität Göttingen.

\section{1 Häufigkeitsverteilung verschiedener Parameter}

Durch die anhand der Akten ermittelten Werte konnten verschiedene Häufigkeiten dargestellt werden. Diese beziehen sich auf demographische Daten wie Alter und Geschlecht sowie die Prothesenverteilung. Außerdem wurden Ausgangsbefunde wie die Topographie, Anzahl, Vitalität und der Lockerungsgrad der einbezogenen Pfeilerzähne sowie die Art der jeweiligen antagonistischen Versorgung erfasst. 


\subsubsection{Geschlechterverteilung}

Es wurden 221 Patienten, davon 100 Frauen und 121 Männer, in die Studie einbezogen.

Es konnte insgesamt ein Durchschnittsalter zum Insertionszeitpunkt von 62,3 Jahren ermittelt werden. Das Durchschnittsalter betrug bei Frauen 62,8 Jahre und bei Männern 61,5 Jahre (Abb. 4).

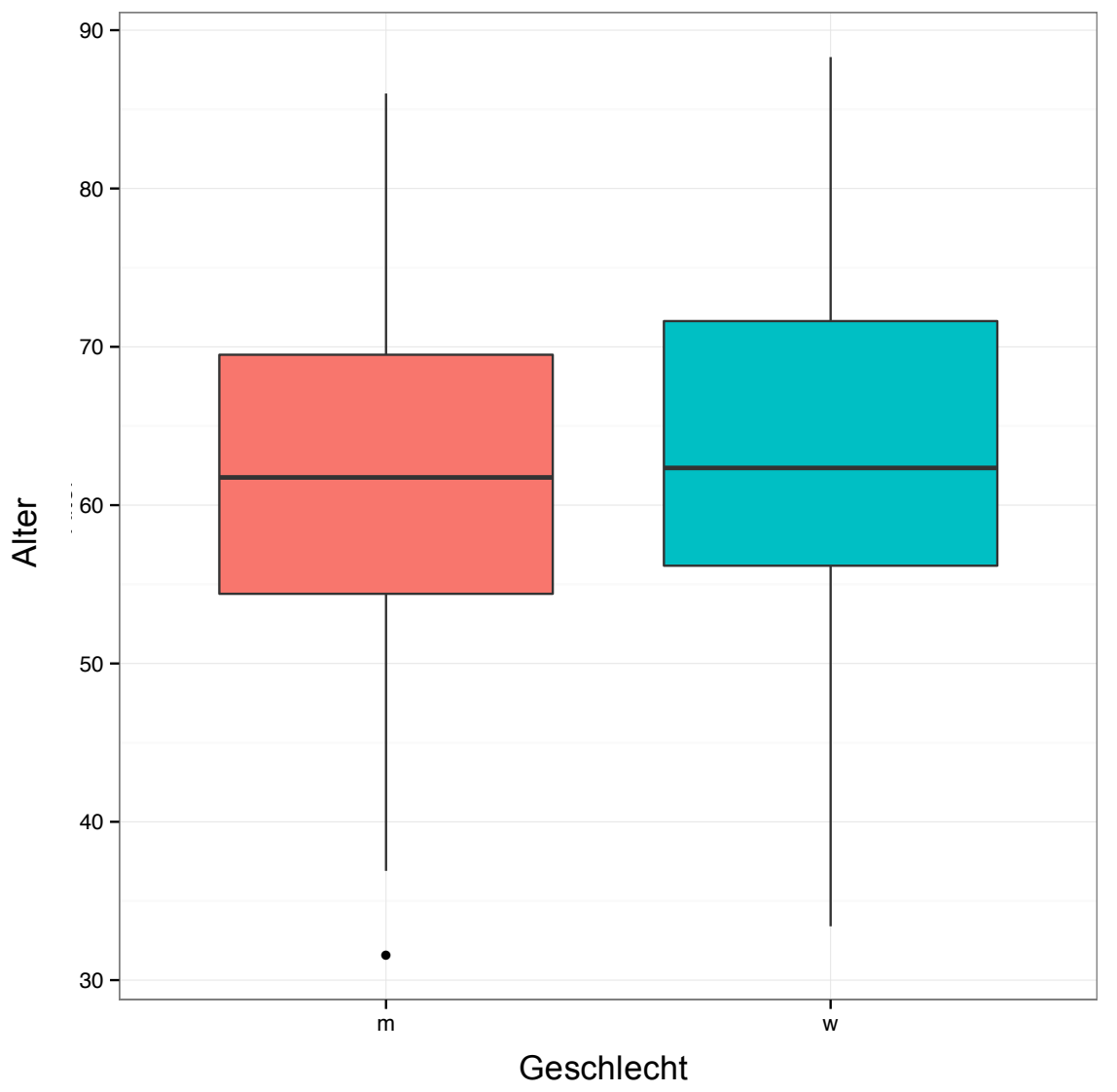

Abb. 4: Geschlechterspezifische Altersverteilung der 221 eingeschlossenen Patienten mittels Boxplot-Diagramm 


\subsubsection{Prothesenverteilung}

221 Patienten wurden mit insgesamt 263 Prothesen, davon 136 im Unterkiefer und 127 im Oberkiefer, versorgt (Abb. 5).

116 Versorgungen wurden bei weiblichen Patientinnen und 147 bei männlichen Patienten eingesetzt.

16 weibliche und 26 männliche Patienten wurden mit einer Ober- und Unterkiefer Resilienzteleskopprothese versorgt. 179 Patienten, davon 84 Frauen und 95 Männer, erhielten eine Resilienzteleskopprothese im Ober- oder Unterkiefer.

Im Falle einer Mehrfachversorgung im Laufe der Zeit wurde bei allen Patienten jeweils nur die erste Versorgung ab dem Jahr 2000 sowohl im Oberkiefer als auch im Unterkiefer aufgenommen.

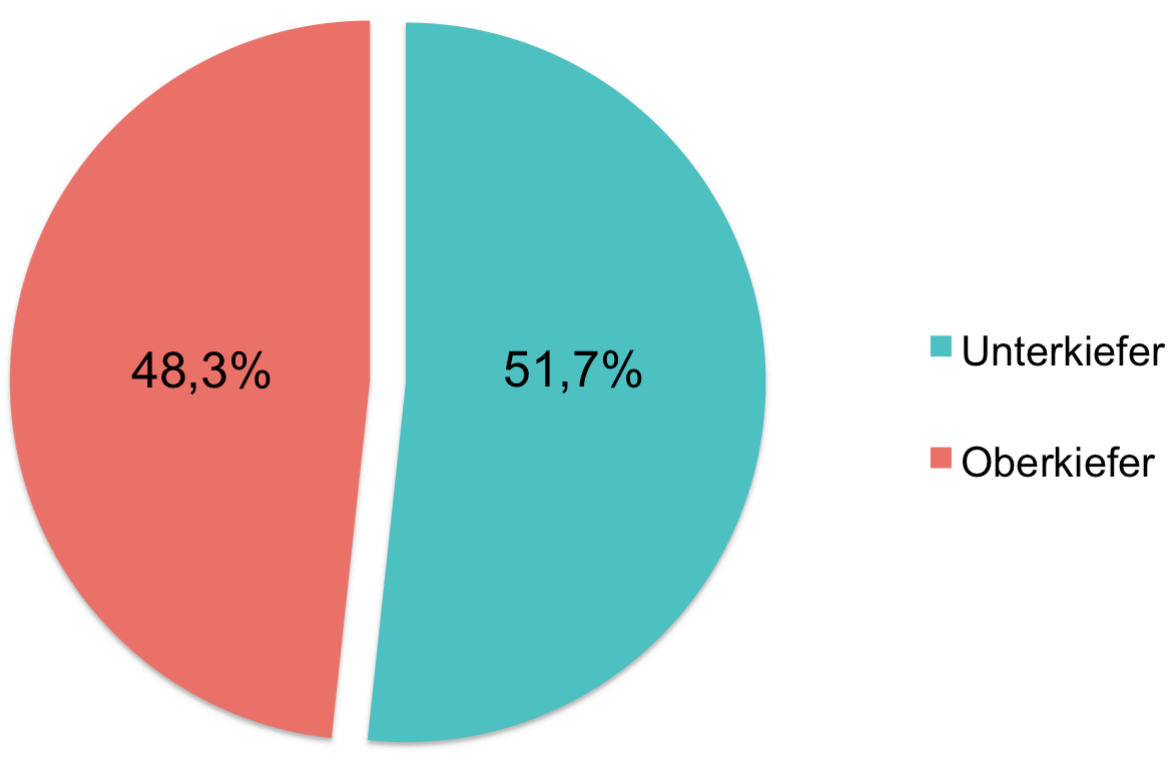

Abb. 5: Verteilung der 263 Resilienzteleskopprothesen im Ober- und Unterkiefer 


\subsubsection{Topographie und Anzahl der Pfeilerzähne}

263 Resilienztelekopprothesen wurden auf insgesamt 538 Pfeilerzähnen verankert. Dabei wurde die Verteilung der jeweiligen Teleskoppfeiler vermerkt und ausgewertet. Aus der nachfolgend erstellten Abbildung 6 wird ersichtlich, dass der Eckzahn in allen Quadranten am häufigsten als Pfeilerzahn in einer Resilienzteleskopversorgung einbezogen wurde. Im Oberkiefer schließen sich zur Verankerung vor allem die übrigen Frontzähne an, im Unterkiefer eher die Prämolaren.

Am häufigsten (101 Fälle) wurden in dieser Studie Resilienzteleskopprothesen mit zwei Pfeilerzähnen eingesetzt. 87 Versorgungen wurden über drei Pfeilerzähne und 75 Prothesen über einen Pfeilerzahn abgestützt.

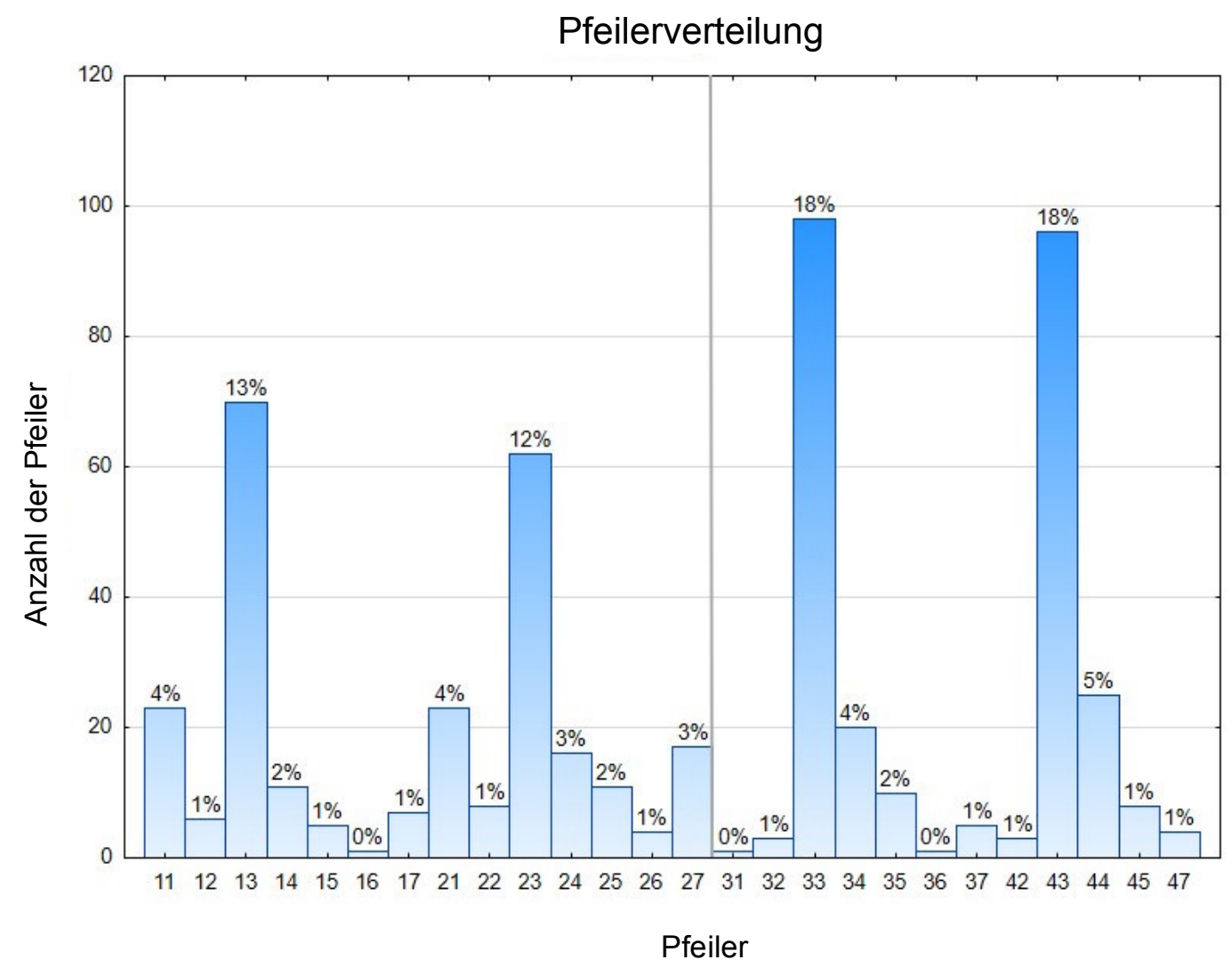

Abb. 6: Pfeilerverteilung der 263 Prothesen im Ober- und Unterkiefer (insgesamt 538 Pfeilerzähne) 


\subsubsection{Vitalität und Lockerungsgrad der Pfeilerzähne}

Zum Eingliederungszeitpunkt der Resilienzteleskopprothesen wurde die Vitalität und der Lockerungsgrad von dem behandelnden Zahnarzt in der Akte notiert und anschließend in dieser Studie aufgenommen. Leider konnte nicht immer ein Wert für diesen Parameter in der Akte gefunden werden, so dass es ebenfalls einen Anteil an fehlenden Werten („Missings“) gibt.

485 Pfeilerzähne waren zum Eingliederungszeitpunkt vital und 48 devital. Bei 5 Pfeilern konnte retrospektiv die Vitalität nicht ermittelt werden.

Nach der Kaplan-Meier-Analyse betrug nach einer Beobachtungszeit von 5 Jahren, die Vitalitätsrate $82 \%$. Nach 8 und 10 Jahren ließ sich eine Wahrscheinlichkeit von $73 \%$ ermitteln. Ein statistisch signifikanter Unterschied stellte sich in der CoxRegressionsanalyse nicht dar.

129 der untersuchten Pfeilerzähne wiesen zum Insertionszeitpunkt einen Lockerungsgrad von 0 auf, 109 einen Lockerungsgrad von 1 und 32 einen Lockerungsgrad von 2. Nur 1 Pfeilerzahn hatte zum Eingliederungszeitpunkt einen Lockerungsgrad von 3. Bei 267 Pfeilerzähnen wurde diese Information nicht in der Akte vermerkt. 


\subsubsection{Antagonistische Versorgung}

Bei allen 263 Resilienzteleskopprothesen konnte die Art der Gegenbezahnung ermittelt und dokumentiert werden (Tab. 1). Daraus ergab sich, dass 168 antagonistische Versorgungen einem kombiniert festsitzend-abnehmbaren Zahnersatz entsprachen. Dieser setzte sich aus verschiedenen Untergruppen zusammen. Dazu zählten teleskopierende- sowie Interimsversorgungen, Klammer-Modellgussprothesen, implantatgetragene Totalprothesen sowie Resilienzteleskopprothesen.

69 Patienten waren antagonistisch mit Totalprothesen versorgt.

Bei 26 Patienten konnte im Gegenkiefer eine festsitzende Versorgung in Form von Brücken, Kronen oder einer rein natürlichen Bezahnung festgestellt werden.

Häufigkeitstabelle Antagonistische Versorgung

\begin{tabular}{lll}
\hline Versorgung & Anzahl & Prozent \\
\hline $\begin{array}{l}\text { Kombiniert festsitzend- } \\
\text { abnehmbarer Zahnersatz }\end{array}$ & 168 & 63,9 \\
Totalprothesen & 69 & \\
Festsitzender Zahnersatz & 26 & 26,2 \\
\hline
\end{tabular}

Tab.1: Art und Verteilung der antagonistischen Versorgung bei den 263 Resilienzteleskopprothesen 


\subsection{Ereigniszeitanalysen der Resilienzteleskopprothesen nach Kaplan-Meier}

Anhand verschiedener Parameter wurden Ereigniszeitanalysen nach Kaplan-Meier durchgeführt und anschließend ausgewertet.

Bei allen statistischen Tests wurde ein Signifikanzniveau von $5 \%$ festgelegt. Alle Analysen wurden durch die Statistik Software R mit Hilfe des R-Paketes 'prodlim' durchgeführt. Alle Grafen wurden jeweils mit drei gestrichelten Linien markiert, welche die Beobachtungszeit nach 5, 8 und 10 Jahren verdeutlichten.

\subsection{1 Überlebenswahrscheinlichkeit}

Es konnte anhand der Kaplan-Meier-Analyse insgesamt eine Überlebenswahrscheinlichkeit der Resilienzteleskopprothesen von 62\% nach 5 Jahren (95\% Konfidenzintervall: $[0.55 ; 0.69]), 38 \%$ nach 8 Jahren (95\% Konfidenzintervall: [0.3; $0.45])$ und $28 \%$ nach 10 Jahren (95\% Konfidenzintervall: $[0.2 ; 0.36])$ festgestellt werden (Abb. 7).

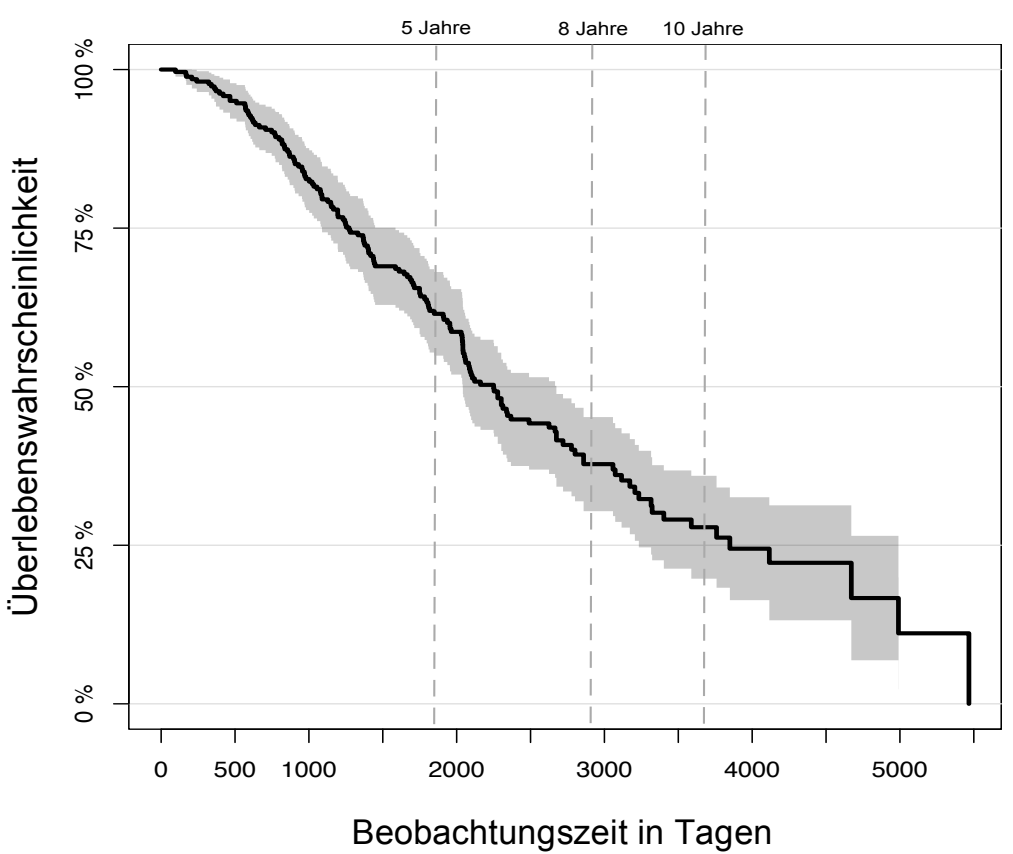

Abb.7: Zeitabhängige Überlebenswahrscheinlichkeit (nach Kaplan-Meier) der 263 Resilienzteleskopprothesen 


\subsubsection{Erfolgswahrscheinlichkeit (keine Komplikation/ Intervention)}

Die Erfolgswahrscheinlichkeit für die untersuchten Resilienzteleskopprothesen betrug nach 5 Jahren 13\% (95\% Konfidenzintervall: [0.09; 0.17]).

Nach einem Zeitraum von 8 Jahren wurde nur noch eine Erfolgswahrscheinlichkeit von 3\% (95\% Konfidenzintervall: $[0.01 ; 0.05])$ und nach 10 Jahren von 1\% (95\% Konfidenzintervall: [0; 0.03]) ermittelt (Abb. 8).

Alle Komplikationen, ausgenommen biologische Prozesse (außer Druckstellen), wurden erst nach 6 Monaten als solche gewertet und aufgenommen. Zuvor aufgetretene technische Komplikationen waren dem Adaptationsgeschehen nach Eingliederung der jeweiligen Prothese zuzurechnen.

In vergleichbaren Untersuchungen wurden Kontrollen bezüglich der Langzeitbewährung von teleskopverankerten Prothesen ebenfalls zunächst wenige Tage nach der Eingliederung (Basline) und danach halbjährlich durchgeführt (Stark und Schrenker 1998).

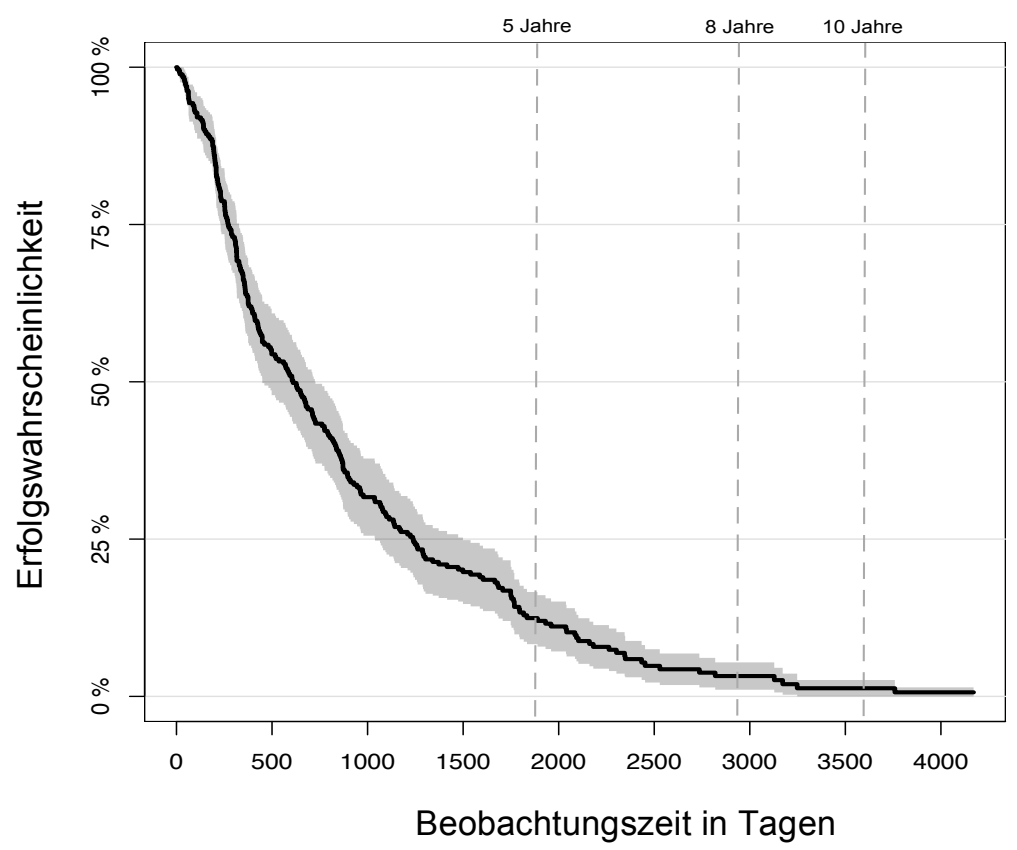

Abb. 8: Zeitabhängige Erfolgswahrscheinlichkeit (nach Kaplan-Meier) der untersuchten Resilienzteleskopprothesen 


\subsubsection{Pfeilerüberlebensrate}

Die Kaplan-Meier-Analyse ergab, dass die Überlebenswahrscheinlichkeit eines Pfeilerzahnes nach einer Zeitspanne von 5 Jahren 55\% und nach 8 Jahren 34\% betrug. Nach einer Beobachtungszeit von 10 Jahren konnte ein Pfeilerverlust mit einer Wahrscheinlichkeit von 30\% ausgeschlossen werden (Abb. 9).

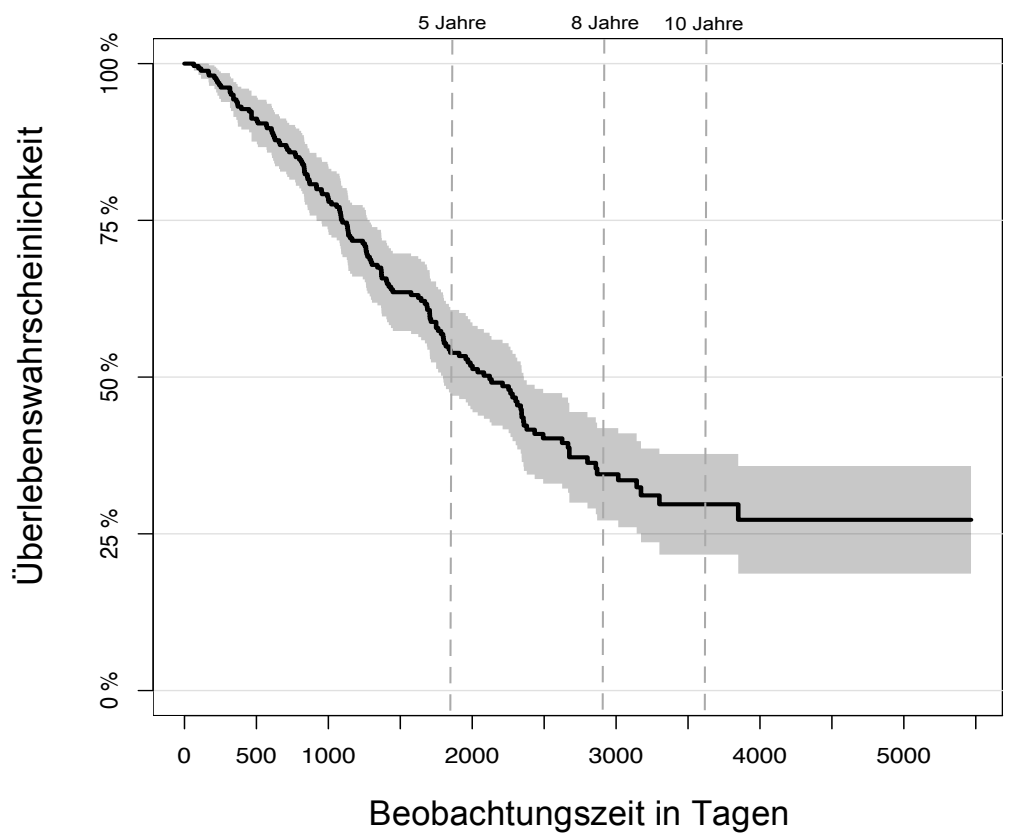

Abb. 9: Zeitabhängige Pfeilerüberlebensrate (nach Kaplan-Meier) der 263 Resilienzteleskopprothesen auf 538 Pfeilerzähnen 


\subsubsection{Verlust durch Pfeilerfraktur}

Nach einer Beobachtungszeit von 5 Jahren konnte ein frakturfreies Überleben der Pfeilerzähne mit einer Wahrscheinlichkeit von 76\% festgestellt werden. Nach 8 und 10 Jahren konnten nur noch Wahrscheinlichkeiten von $69 \%$ bzw. $64 \%$ ermittelt werden (Abb. 10).

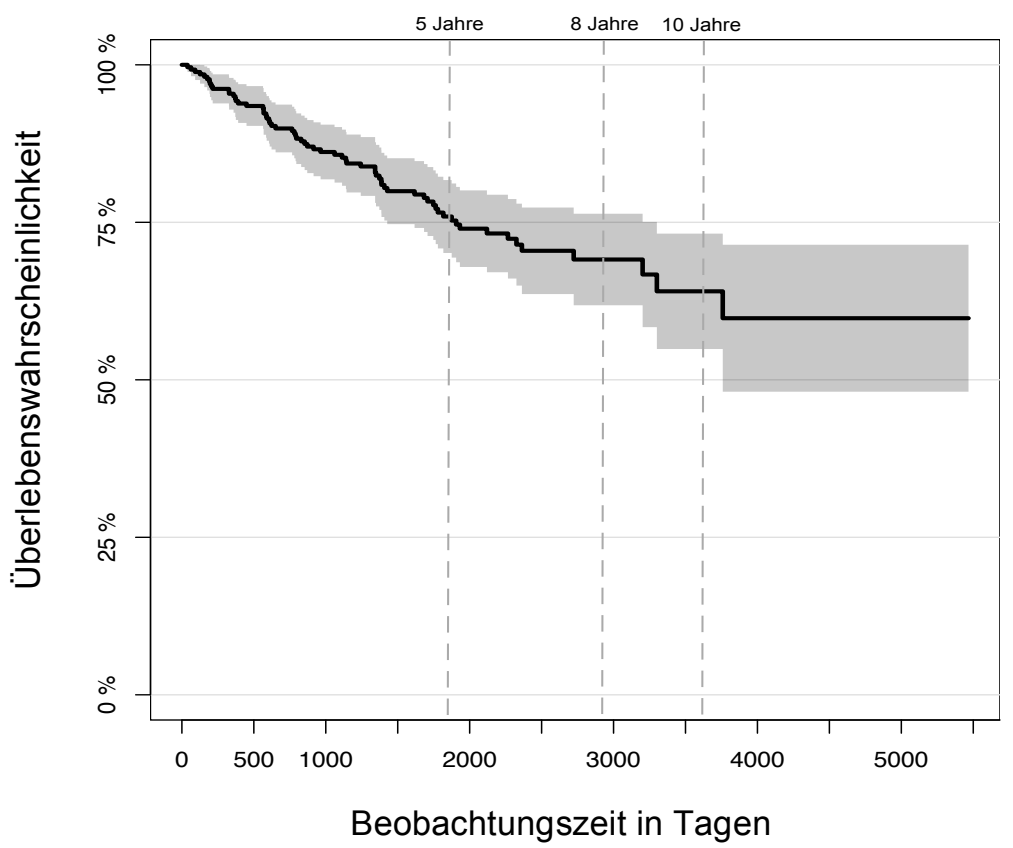

Abb. 10: Zeitabhängige Überlebensrate (nach Kaplan-Meier) der 538 Pfeilerzähne bezogen auf den Verlust durch Pfeilerfraktur 


\subsubsection{Biologische Erfolgsrate}

Anhand der Kaplan-Meier-Analyse konnten biologische Komplikationen nach 5 Jahren zu 44\% ausgeschlossen werden. Nach einen Zeitraum von 8 bzw. 10 Jahren wurden Wahrscheinlichkeiten von $37 \%$ angegeben (Abb. 11). Ein statistisch signifikanter Unterschied zu den Beobachtungszeiten konnte anhand der CoxRegressionsanalyse nicht ermittelt werden.

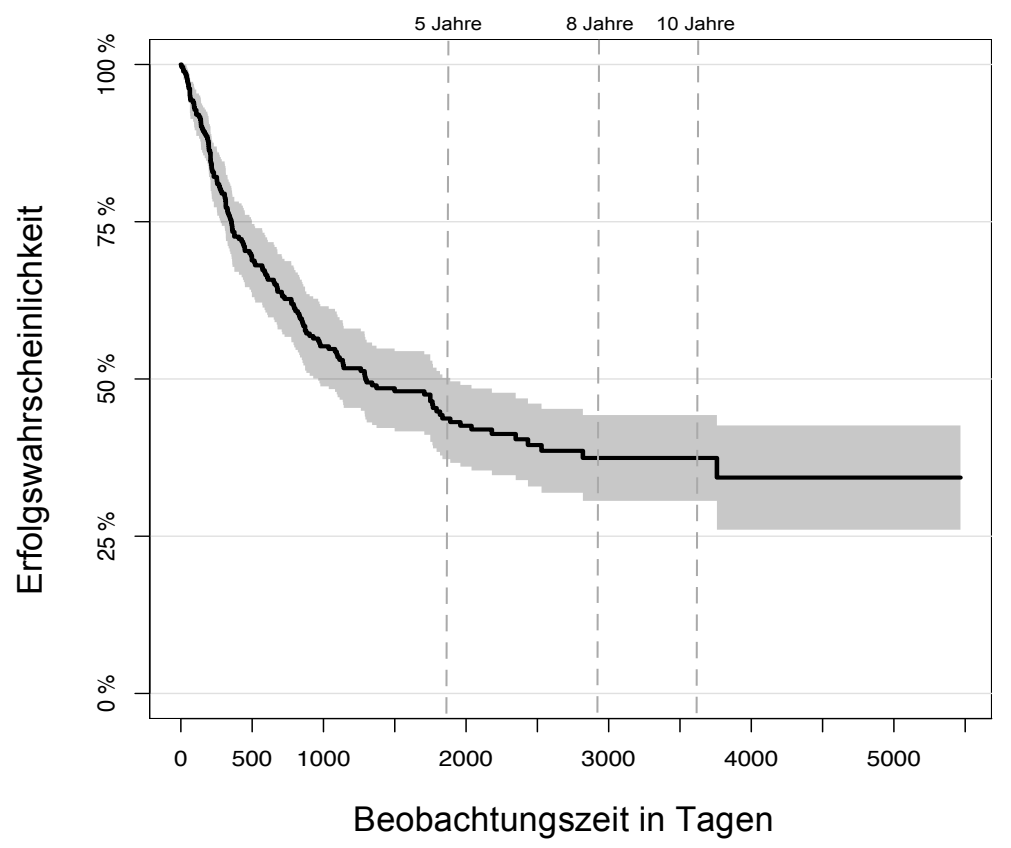

Abb. 11: Zeitabhängige Erfolgswahrscheinlichkeit (nach Kaplan-Meier) bezogen auf biologische Komplikationen 


\subsubsection{Technische Erfolgsrate}

Eine technische Komplikationsfreiheit konnte - nach einem Beobachtungszeitraum von 5 Jahren - mit einer Wahrscheinlichkeit von 63\% festgestellt werden. Nach einem Zeitraum von 8 Jahren ergab sich eine Wahrscheinlichkeit von $53 \%$ und nach 10 Jahren eine Wahrscheinlichkeit von 47\% (Abb. 12). Ein signifikanter Unterschied konnte nicht ermittelt werden.

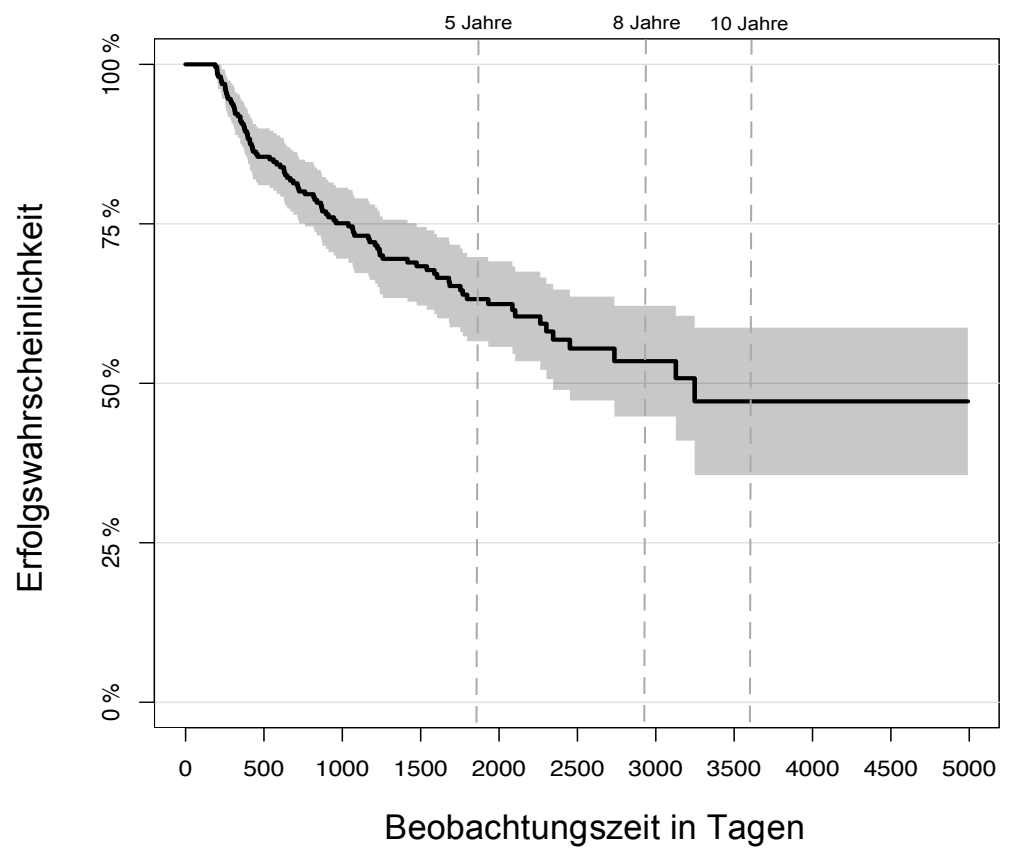

Abb. 12: Zeitabhängige Erfolgswahrscheinlichkeit (nach Kaplan-Meier) bezogen auf technische Komplikationen 


\subsection{Ereigniszeitanalysen in Abhängigkeit von möglichen Risikofaktoren}

\subsubsection{Risikofaktor Pfeileranzahl}

\subsubsection{1 Überlebenswahrscheinlichkeit in Abhängigkeit von der Pfeileranzahl}

Anhand der durchgeführten Analyse nach Kaplan-Meier war zu erkennen, dass die Überlebenswahrscheinlichkeit der Resilienzteleskopprothesen mit zunehmender Anzahl der Pfeilerzähne steigt (Abb. 13).

Nach einer Beobachtungszeit von 5 Jahren wurde anhand der Kaplan-Meier-Analyse eine Überlebenswahrscheinlichkeit von 39\% mit einem Pfeilerzahn, von 63\% mit zwei Pfeilerzähnen und 80\% mit drei Pfeilerzähnen ermittelt.

Nach 8 Jahren betrug die Überlebenswahrscheinlichkeit mit einem Pfeilerzahn 16\%, mit zwei Pfeilerzähnen 27\% und mit drei Pfeilerzähnen $70 \%$.

Es konnte bei Versorgungen mit einem Pfeilerzahn, nach einer Zeitspanne von 10 Jahren, ebenfalls ein Überleben mit einer Wahrscheinlichkeit von $16 \%$ festgestellt werden. Ein nur gering besseres Ergebnis (20\%) konnten Prothesen mit zwei Pfeilerzähnen erreichen, wohingegen sich drei Pfeilerzähne mit 48\% deutlich abgrenzten (Tab. 2).

Anhand der durchgeführten Cox-Regressionsanalyse konnte gezeigt werden, dass das Hazard Ratio bei Resilienzteleskopprothesen mit einem Pfeilerzahn 3,54 und mit zwei Pfeilerzähnen 2,43 betrug (95\% Konfidenzintervall: [2.22; 5.65], [1.59; 3.71]). Es lässt sich somit sagen, dass das Risiko, eine Prothese zu verlieren, bei Versorgungen mit einem Pfeilerzahn 3,54 und mit zwei Pfeilerzähnen 2,43-mal so hoch ist, im Vergleich zur Referenzgruppe mit drei Pfeilerzähnen.

Es konnte ein signifikanter Unterschied $(p<0,001)$ ermittelt werden. 


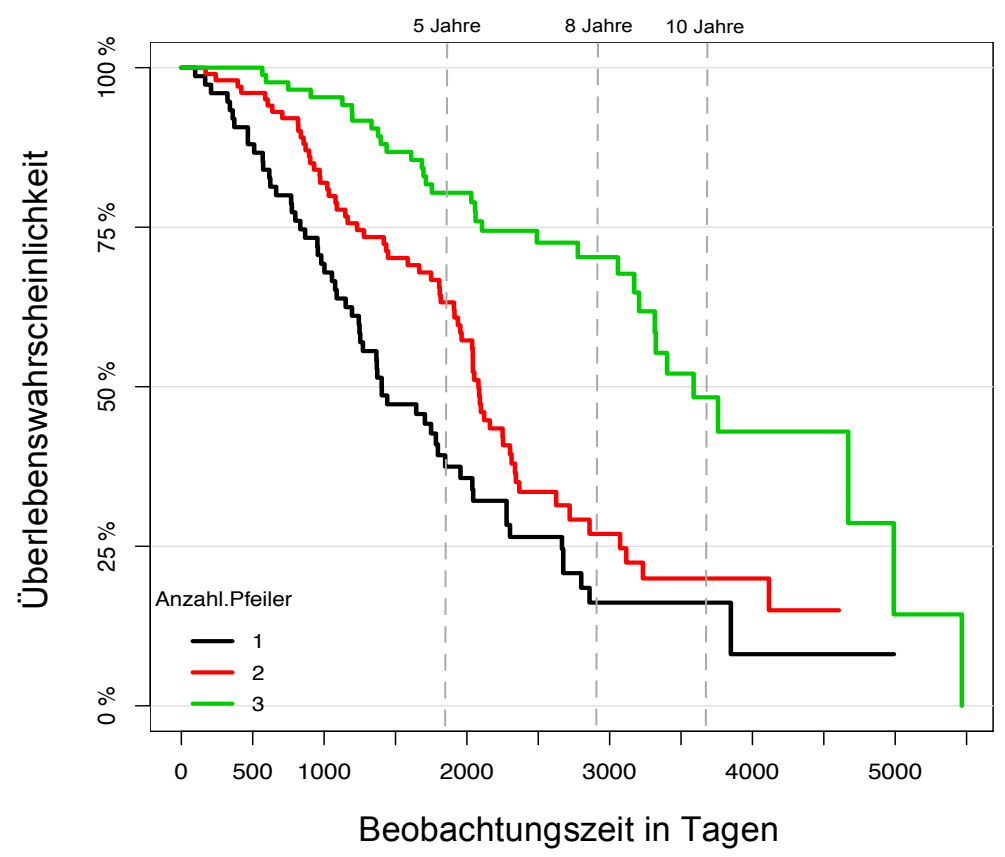

Abb. 13: Zeitabhängige Überlebenswahrscheinlichkeit (nach Kaplan-Meier) der 263 Resilienzteleskopprothesen in Abhängigkeit von der Pfeileranzahl

\begin{tabular}{|c|c|c|c|c|}
\hline Variable & Zeit (Jahre) & 1 Pfeilerzahn & 2 Pfeilerzähne & 3 Pfeilerzähne \\
\hline \multirow{3}{*}{ Überleben } & 5 & $39 \%$ & $63 \%$ & $80 \%$ \\
\cline { 2 - 5 } & 8 & $16 \%$ & $27 \%$ & $70 \%$ \\
\cline { 2 - 5 } & 10 & $16 \%$ & $20 \%$ & $48 \%$ \\
\hline
\end{tabular}

Tab. 2: Zeitabhängige Überlebenswahrscheinlichkeit der 263 Resilienzteleskopprothesen in Abhängigkeit von der Pfeileranzahl 


\subsubsection{Erfolgswahrscheinlichkeit in Abhängigkeit von der Pfeileranzahl}

Die Kaplan-Meier-Analyse demonstrierte, dass Versorgungen mit einem Pfeilerzahn eine Erfolgsrate von lediglich 6\%, mit zwei Pfeilerzähnen von $11 \%$ und mit drei Pfeilerzähnen von $20 \%$ im 5-Jahreszeitraum aufwiesen. Weitere Daten ließen sich nach dieser Zeit leider nur noch für Resilienzteleskopprothesen mit zwei oder drei Pfeilerzähnen ermitteln. Es konnte eine Erfolgsrate von 1\% nach 8 Jahren bei Versorgungen mit zwei Pfeilerzähnen festgestellt werden. Prothesen mit drei Pfeilerzähnen erzielten nach 8 Jahren zu $7 \%$ und nach 10 Jahren zu 3\% eine Komplikationsfreiheit (Abb. 14, Tab. 3).

Anhand der durchgeführten zeitabhängigen Analyse konnte ermittelt werden, dass das Auftreten einer Komplikation bei Resilienzteleskopprothesen mit zwei Pfeilerzähnen im Vergleich zur Referenzgruppe mit drei Pfeilerzähnen 1,4-fach erhöht ist. Dieser Unterschied war statistisch signifikant ( $p=0,0353)$.

Versorgungen mit einem Pfeilerzahn ergaben keinen signifikanten Unterschied bezüglich der Erfolgswahrscheinlichkeit, jedoch konnte eine Tendenz für das erhöhte Auftreten einer Komplikation festgestellt werden $(p=0,0525)$. 


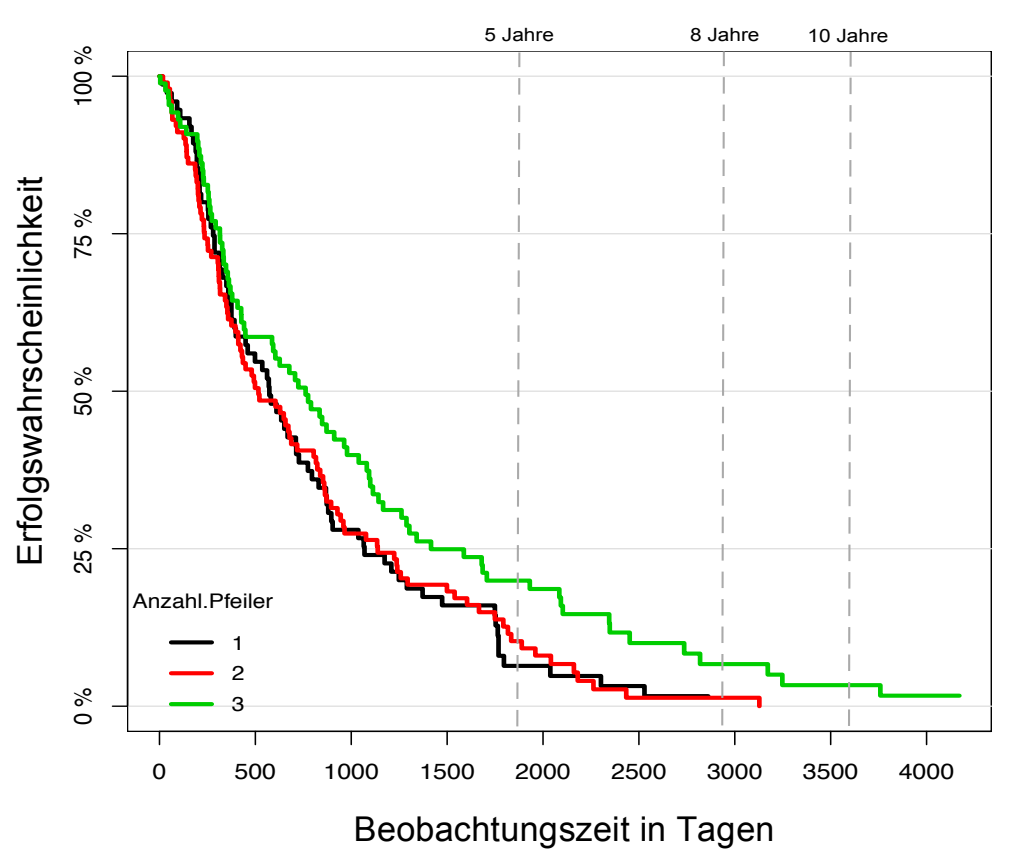

Abb. 14: Zeitabhängige Erfolgswahrscheinlichkeit (nach Kaplan-Meier) der 263 Resilienzteleskopprothesen in Abhängigkeit von der Pfeileranzahl

\begin{tabular}{|c|c|c|c|c|}
\hline Variable & Zeit (Jahre) & 1 Pfeilerzahn & 2 Pfeilerzähne & 3 Pfeilerzähne \\
\hline \multirow{3}{*}{ Erfolg } & 5 & $6 \%$ & $11 \%$ & $20 \%$ \\
\cline { 2 - 5 } & 8 & NA & $1 \%$ & $7 \%$ \\
\cline { 2 - 5 } & 10 & NA & NA & $3 \%$ \\
\hline
\end{tabular}

Tab. 3: Zeitabhängige Erfolgswahrscheinlichkeit der 263 Resilienzteleskopprothesen in Abhängigkeit von der Pfeileranzahl, (NA = Not Available) 


\subsubsection{Pfeilerverlust}

Bei Resilienzteleskopprothesen mit einem Pfeilerzahn ist nach 5 Jahren mit einer Wahrscheinlichkeit von $46 \%$ kein Verlust des Pfeilerzahnes zu erwarten gewesen.

Ein Verlust des Zahnes konnte bei Versorgungen mit zwei Pfeilerzähnen zu 55\% und mit drei Pfeilerzähnen zu 62\% nach gleicher Zeitspanne ausgeschlossen werden.

Nach einem Beobachtungszeitraum von 8 Jahren wurde eine Pfeilerüberlebenswahrscheinlichkeit von $23 \%$ bei Prothesen mit einem Pfeilerzahn und von $53 \%$ bei Resilienzteleskopprothesen mit drei Pfeilerzähnen festgestellt.

Nach 10 Jahren ergab sich eine Pfeilerüberlebenswahrscheinlichkeit von 39\% bei Versorgungen mit drei Pfeilerzähnen. Prothesen mit zwei Pfeilerzähnen erzielten sowohl nach 8 Jahren als auch nach 10 Jahren eine Pfeilerüberlebenswahrscheinlichkeit von $25 \%$. Ähnliches konnte bei Versorgungen mit einem Pfeilerzähnen beobachtet werden. Diese ließen nach 8 und 10 Jahren ebenfalls ein gleiches Ergebnis von 23\% erkennen (Abb. 15, Tab. 4).

Die durchgeführte Cox-Regressionsanalyse ergab bei Versorgungen mit einem Pfeilerzahn im Vergleich zu drei Pfeilerzähnen ein Hazard Ratio von 1,72. Somit konnte ein statistisch signifikanter Unterschied ermittelt werden $(p=0,0207)$. Ähnliche Tendenz, jedoch keinen signifikanten Effekt, zeigten Prothesen mit zwei Pfeilerzähnen relativ zur Referenzgruppe mit drei Pfeilerzähnen $(p=0,0508)$. 


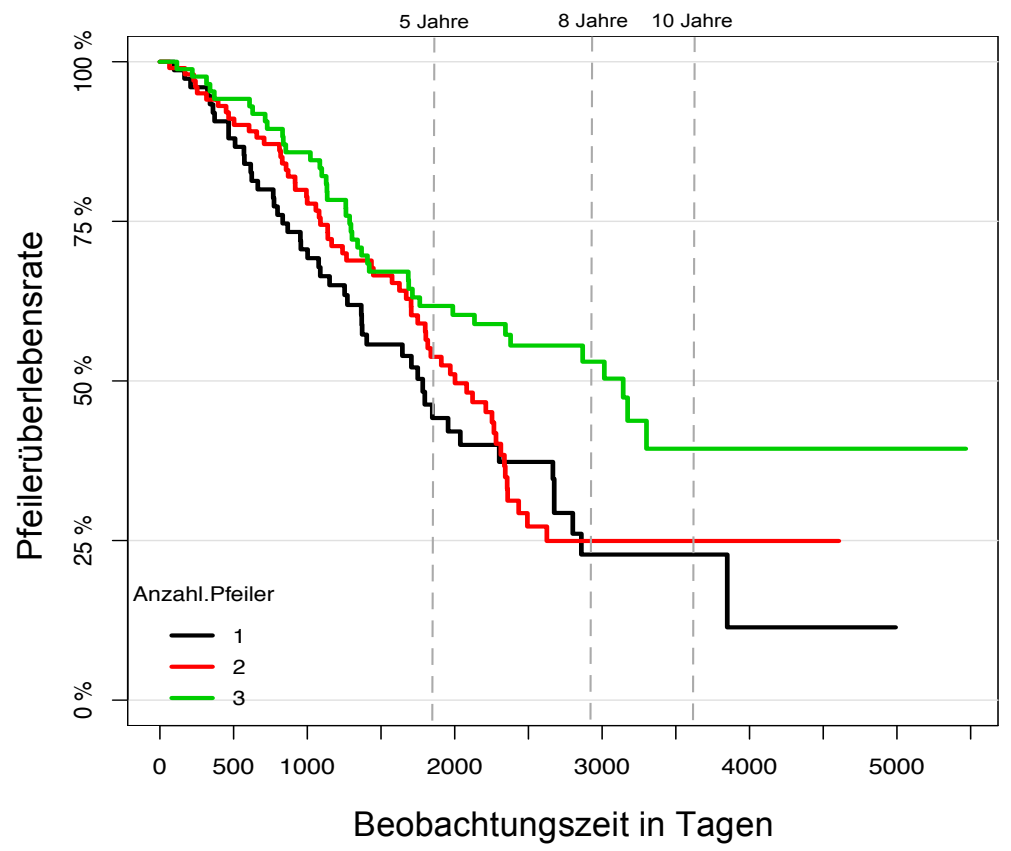

Abb. 15: Zeitabhängige Pfeilerüberlebenswahrscheinlichkeit (nach Kaplan-Meier) der 263 Resilienzteleskopprothesen in Abhängigkeit von der Pfeileranzahl

\begin{tabular}{|c|c|c|c|c|}
\hline Variable & Zeit (Jahre) & 1 Pfeilerzahn & 2 Pfeilerzähne & 3 Pfeilerzähne \\
\hline \multirow{3}{*}{ Pfeilerverlust } & 5 & $46 \%$ & $55 \%$ & $62 \%$ \\
\cline { 2 - 5 } & 8 & $23 \%$ & $25 \%$ & $53 \%$ \\
\cline { 2 - 5 } & 10 & $23 \%$ & $25 \%$ & $39 \%$ \\
\hline
\end{tabular}

Tab. 4: Zeitabhängige Pfeilerüberlebenswahrscheinlichkeit der 263 Resilienzteleskopprothesen bezüglich Pfeilerverlust 


\subsubsection{Pfeilerfraktur}

Aus der Kaplan-Meier-Analyse ging hervor, dass bei Versorgungen mit drei Pfeilerzähnen mit einer Wahrscheinlichkeit von 83\% nach 5 Jahren keine Fraktur aufgetreten ist. Resilienzteleskopprothesen mit zwei Pfeilerzähnen zeigten nach dieser Zeit zu 79\% und mit einem Pfeilerzahn zu 63\% keine Fraktur. Nach einem Beobachtungszeitraum von 8 Jahren konnte bei Versorgungen mit drei Pfeilerzähnen eine Wahrscheinlichkeit von 75\%, mit zwei Pfeilerzähnen von $71 \%$ und einem Pfeilerzahn von 59\% festgestellt werden. Die gleiche Wahrscheinlichkeit konnte nach 10 Jahren bei Prothesen mit einem oder zwei Pfeilerzähnen beobachtet werden. Versorgungen mit drei Pfeilerzähnen erzielten nach dieser Zeit 67\% (Abb. 16, Tab. 5).

Die Untersuchung der abhängigen Variablen Pfeilerfraktur zeigte einen statistisch signifikanten Unterschied zwischen Resilienzteleskopprothesen mit einem Pfeilerzahn im Vergleich zur Referenzgruppe mit drei Pfeilerzähnen $(p=0,0249)$. Bei Versorgungen mit zwei Pfeilerzähnen konnte keine statistische Signifikanz festgestellt werden.

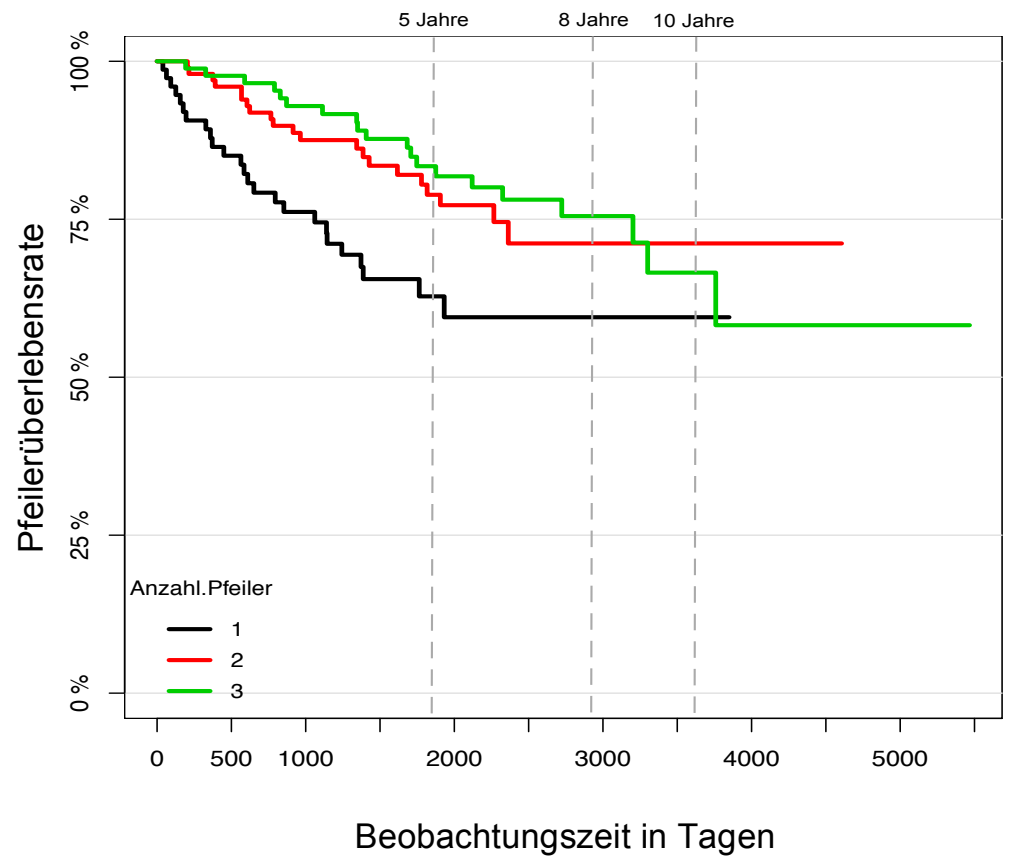

Abb. 16: Zeitabhängige Pfeilerüberlebenswahrscheinlichkeit (keine Fraktur) (nach Kaplan-Meier) der 263 Resilienzteleskopprothesen auf 538 Pfeilerzähnen in Abhängigkeit von der Pfeileranzahl 


\begin{tabular}{|c|c|c|c|c|}
\hline Variable & Zeit (Jahre) & 1 Pfeilerzahn & 2 Pfeilerzähne & 3 Pfeilerzähne \\
\hline \multirow{3}{*}{ Pfeilerfraktur } & 5 & $63 \%$ & $79 \%$ & $83 \%$ \\
\cline { 2 - 5 } & 8 & $59 \%$ & $71 \%$ & $75 \%$ \\
\cline { 2 - 5 } & 10 & $59 \%$ & $71 \%$ & $67 \%$ \\
\hline
\end{tabular}

Tab. 5: Zeitabhängige Pfeilerüberlebenswahrscheinlichkeit (keine Fraktur) der 263 Resilienzteleskopprothesen 


\subsubsection{Erfolgswahrscheinlichkeit bezogen auf Retentionsverlust}

Die Kaplan-Meier-Analyse ergab eine Wahrscheinlichkeit von $79 \%$ bei Resilienzteleskopprothesen mit einem Pfeilerzahn in einem Intervall von 5,8 und 10 Jahren, dass es zu keinem Verlust der Retention kam. Versorgungen mit zwei Pfeilerzähnen wiesen eine Wahrscheinlichkeit von $81 \%$ nach 5 Jahren und von $70 \%$ nach 8 bzw. 10 Jahren auf. Für Prothesen mit drei Pfeilerzähnen konnte nach 5 Jahren eine Wahrscheinlichkeit von $90 \%$, nach 8 Jahren von $88 \%$ und nach 10 Jahren von $83 \%$ festgestellt werden (Abb. 18, Tab. 6).

Die Cox-Regressionsanalyse ergab bei Versorgungen mit einem Pfeilerzahn ein Hazard Ratio von 2,28 sowie einen p-Wert von 0,0471. Ein statistisch signifikanter Unterschied, im Vergleich zur Referenzgruppe mit drei Pfeilerzähnen, konnte ebenfalls bei Resilienzteleskopprothesen mit zwei Pfeilerzähnen festgestellt werden $(p=0,0277)$.

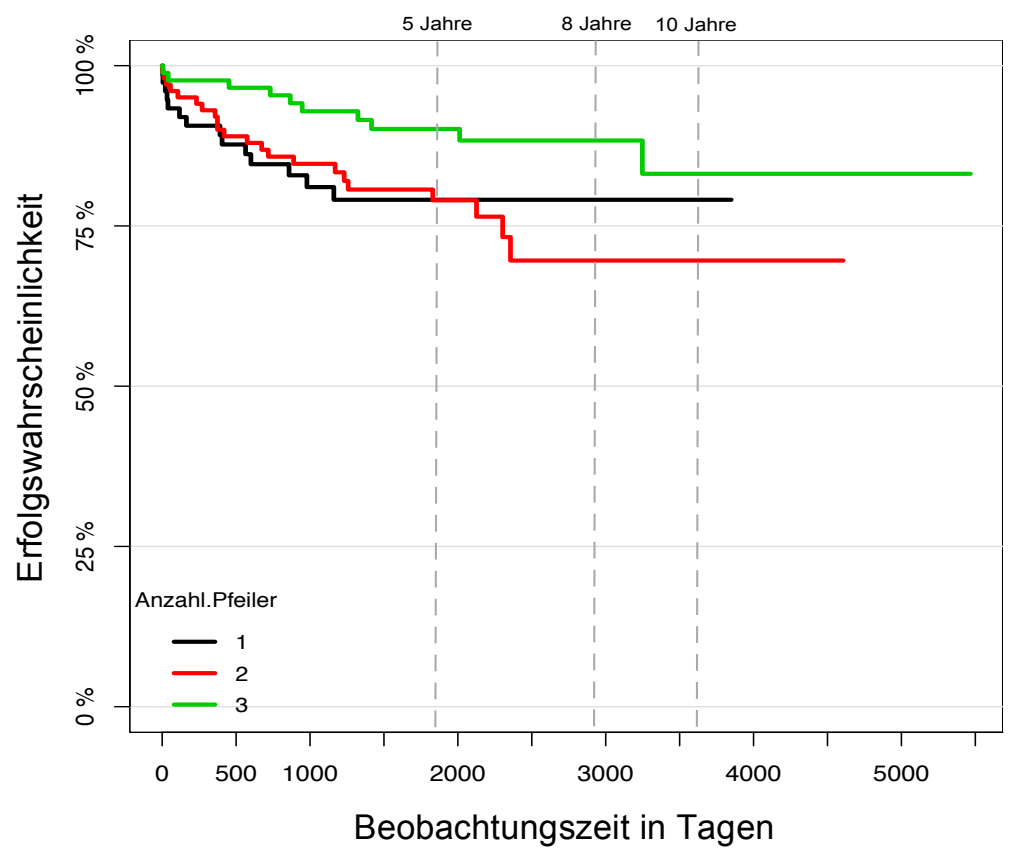

Abb. 18: Zeitabhängige Erfolgswahrscheinlichkeit (nach Kaplan-Meier) der 263 Resilienzteleskopprothesen bezogen auf Retentionsverlust in Anhängigkeit von der Pfeileranzahl 


\begin{tabular}{|c|c|c|c|c|}
\hline Variable & $\begin{array}{c}\text { Zeit } \\
\text { (Jahre) }\end{array}$ & 1 Pfeilerzahn & 2 Pfeilerzähne & 3 Pfeilerzähne \\
\hline \multirow{3}{*}{ Retentionsverlust } & 5 & $79 \%$ & $81 \%$ & $90 \%$ \\
\cline { 2 - 5 } & 8 & $79 \%$ & $70 \%$ & $88 \%$ \\
\cline { 2 - 5 } & 10 & $79 \%$ & $70 \%$ & $83 \%$ \\
\hline
\end{tabular}

Tab. 6: Zeitabhängige Erfolgswahrscheinlichkeit der 263 Resilienzteleskopprothesen bezüglich Retentionsverlust 


\subsubsection{Risikofaktor Antagonistische Versorgung}

\subsubsection{1 Überlebenswahrscheinlichkeit in Abhängigkeit von der antagonistischen Versorgung}

Dargestellt ist die zeitabhängige Überlebenswahrscheinlichkeit der Resilienzteleskopprothesen in Abhängigkeit von der jeweiligen Gegenkieferversorgung. Anhand der aufgeführten Grafik in Abbildung 19 ist zu erkennen, dass sowohl antagonistischer festsitzender Zahnersatz mit einer Überlebenswahrscheinlichkeit von $66 \%$ nach 5 Jahren als auch antagonistischer kombiniert festsitzendabnehmbarer Zahnersatz mit einer Wahrscheinlichkeit von 67\% nach gleicher Zeit zu ähnlichen Langzeitergebnissen führt. Die Überlebenswahrscheinlichkeit bei antagonistischer totalprothetischer Versorgung zeigt einen steileren Abfall des Graphen und konnte nach 5 Jahren mit 49\% dokumentiert werden.

Nach einer Beobachtungszeit von 8 Jahren betrug die Überlebenswahrscheinlichkeit der Resilienzteleskopprothesen bei antagonistischem festsitzenden Zahnersatz 48\%, bei kombiniert festsitzend-abnehmbarem Zahnersatz $46 \%$ und bei Totalprothesen 14\%. Nur noch die Hälfte der Wahrscheinlichkeit ließ sich bei totalprothetischen antagonistischen Versorgungen nach einer Zeitspanne von 10 Jahren feststellen (7\%). Nach dieser Zeit erwies der festsitzende Zahnersatz eine Überlebenswahrscheinlichkeit von 38\% und kombiniert festsitzend-abnehmbarer Zahnersatz von 35\% (Tab. 7).

Die Referenzgruppe festsitzender Zahnersatz wurde jeweils im Zusammenhang mit den Variablen kombiniert festsitzend-abnehmbarer Zahnersatz und Totalprothesen untersucht. Dabei konnte kein signifikanter Unterschied bezüglich der Überlebenszeit festgestellt werden. 


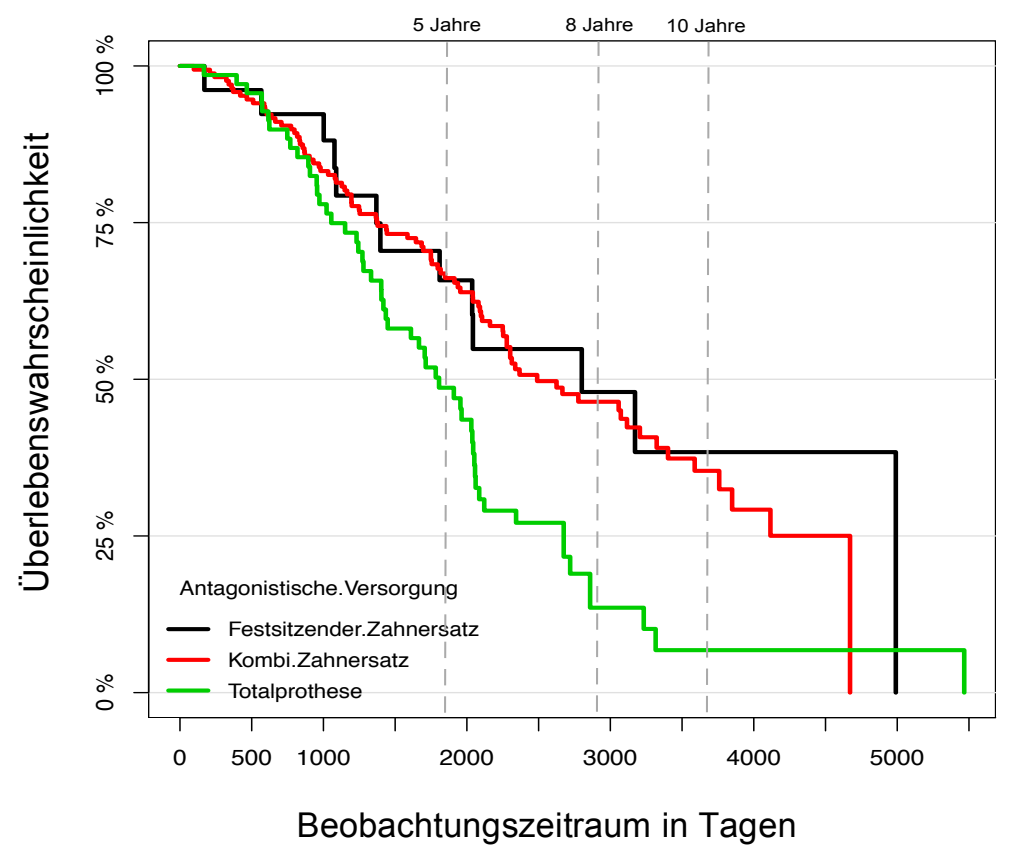

Abb. 19: Zeitabhängige Überlebenswahrscheinlichkeit (nach Kaplan-Meier) der 263 Resilienzteleskopprothesen in Abhängigkeit von der antagonistischen Versorgung

\begin{tabular}{|c|c|c|c|c|}
\hline Variable & $\begin{array}{c}\text { Zeit } \\
\text { (Jahre) }\end{array}$ & $\begin{array}{c}\text { Festsitzender } \\
\text { Zahnersatz }\end{array}$ & $\begin{array}{c}\text { Kombi- } \\
\text { Zahnersatz }\end{array}$ & Totalprothesen \\
\hline \multirow{3}{*}{ Überleben } & 5 & $66 \%$ & $67 \%$ & $49 \%$ \\
\cline { 2 - 5 } & 8 & $48 \%$ & $46 \%$ & $14 \%$ \\
\cline { 2 - 5 } & 10 & $38 \%$ & $35 \%$ & $7 \%$ \\
\hline
\end{tabular}

Tab. 7: Zeitabhängige Überlebenswahrscheinlichkeit der 263 Resilienzteleskopprothesen in Abhängigkeit von der antagonistischen Versorgung 


\subsubsection{Erfolgswahrscheinlichkeit in Abhängigkeit von der antagonistischen Versorgung}

In der Kaplan-Meier-Analyse offenbarten sich tendenziell ähnliche Langzeitergebnisse wie in der zuvor beschriebenen Überlebenszeitanalyse. Die Grafik ist in Abbildung 20 dargestellt.

Bei antagonistischem festsitzenden Zahnersatz wurde nach 5 Jahren für Resilienzteleskopprothesen eine Erfolgswahrscheinlichkeit von 25\% ermittelt. Bei kombiniert festsitzend-abnehmbarem Zahnersatz wurde eine Erfolgswahrscheinlichkeit von 13\% und $8 \%$ für antagonistische Totalprothesen nach dieser Zeit dokumentiert.

Nach einer Beobachtungszeit von 8 Jahren zeigte sich bei antagonistischem festsitzenden Zahnersatz eine Erfolgswahrscheinlichkeit von 8\% und bei kombiniert festsitzend-abnehmbarem Zahnersatz von 4\%. Für antagonistische totalprothetische Versorgungen konnten für 8 und 10 Jahre keine Daten ermittelt werden. Relativ geringe Erfolgswahrscheinlichkeiten von $4 \%$ bei antagonistischem festsitzenden Zahnersatz und $1 \%$ bei kombiniert festsitzend-abnehmbarem Zahnersatz ließen sich nach einer Zeit von 10 Jahren feststellen (Tab. 8).

Es konnte kein signifikanter Einfluss verschiedener antagonistischer Versorgungen auf die Erfolgswahrscheinlichkeit der Resilienzteleskopprothesen festgestellt werden. 


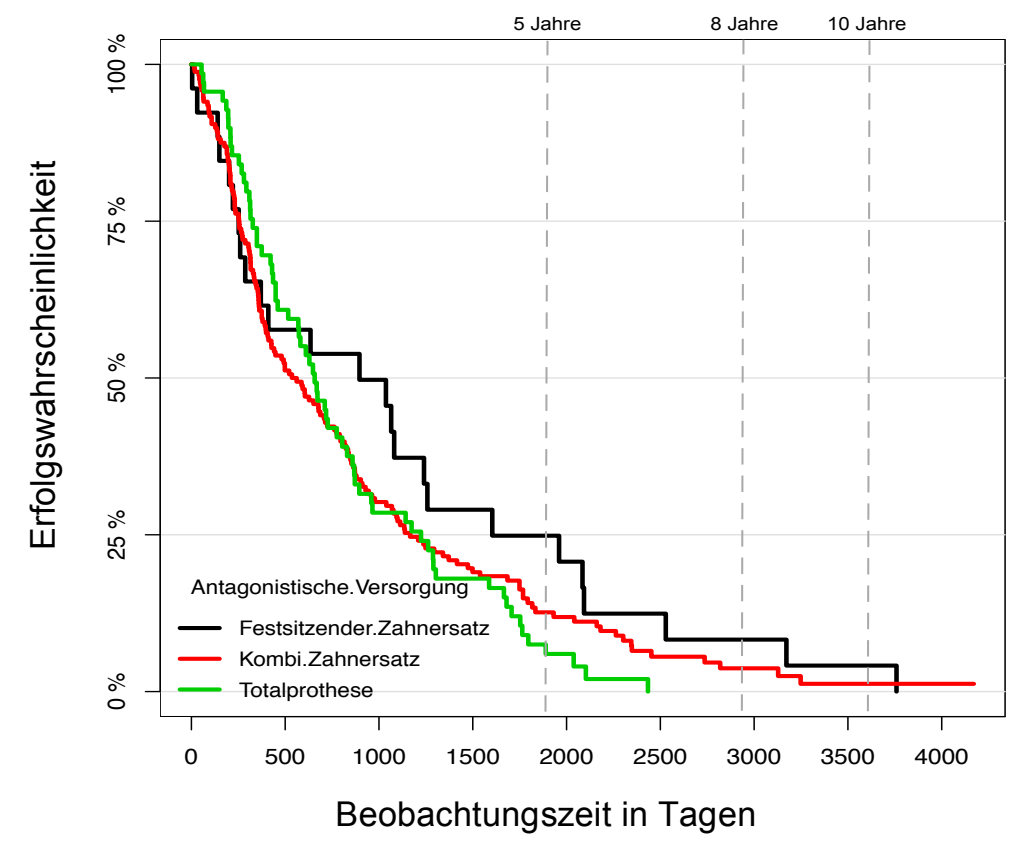

Abb. 20: Zeitabhängige Erfolgswahrscheinlichkeit (nach Kaplan-Meier) der 263 Resilienzteleskopprothesen in Abhängigkeit von der antagonistischen Versorgung

\begin{tabular}{|c|c|c|c|c|}
\hline Variable & $\begin{array}{c}\text { Zeit } \\
\text { (Jahre) }\end{array}$ & $\begin{array}{c}\text { Festsitzender } \\
\text { Zahnersatz }\end{array}$ & $\begin{array}{c}\text { Kombi- } \\
\text { Zahnersatz }\end{array}$ & Totalprothesen \\
\hline \multirow{3}{*}{ Erfolg } & 5 & $25 \%$ & $13 \%$ & $8 \%$ \\
\cline { 2 - 5 } & 8 & $8 \%$ & $4 \%$ & NA \\
\cline { 2 - 5 } & 10 & $4 \%$ & $1 \%$ & NA \\
\hline
\end{tabular}

Tab. 8: Zeitabhängige Erfolgswahrscheinlichkeit der 263 Resilienzteleskopprothesen in Abhängigkeit von der antagonistischen Versorgung, (NA = Not Available) 


\subsubsection{Pfeilerfraktur}

Anhand der durchgeführten Kaplan-Meier-Analyse ließ sich bei der antagonistischen Versorgung festsitzender Zahnersatz nach 5 sowie 8 Jahren zu $75 \%$ keine Pfeilerfraktur bei Resilienzteleskopprothesen feststellen. Bei der antagonistischen Versorgung kombiniert festsitzend-abnehmbarer Zahnersatz waren nach 5 Jahren zu $80 \%$ und nach 8 Jahren zu 74\% keine Pfeilerfrakturen nachweisbar. Totalprothesen als antagonistische Versorgung ergaben nach 5 Jahren eine Wahrscheinlichkeit von $64 \%$ und nach 8 sowie 10 Jahren 54\%. Kombiniert festsitzend-abnehmbarer Zahnersatz zeigte nach 10 Jahren das beste Ergebnis bezüglich einer nicht zu erwartenden Fraktur der Pfeilerzähne bei Resilienzteleskopprothesen (70\%). Die relativ schlechteste Prognose von 50\% wurde bei antagonistischem festsitzenden Zahnersatz für die gleiche Zeit ermittelt (Abb. 21, Tab. 9).

Eine statistische Signifikanz ließ sich bei der antagonistischen Versorgung bezüglich einer zu erwartenden Pfeilerfraktur nicht feststellen.

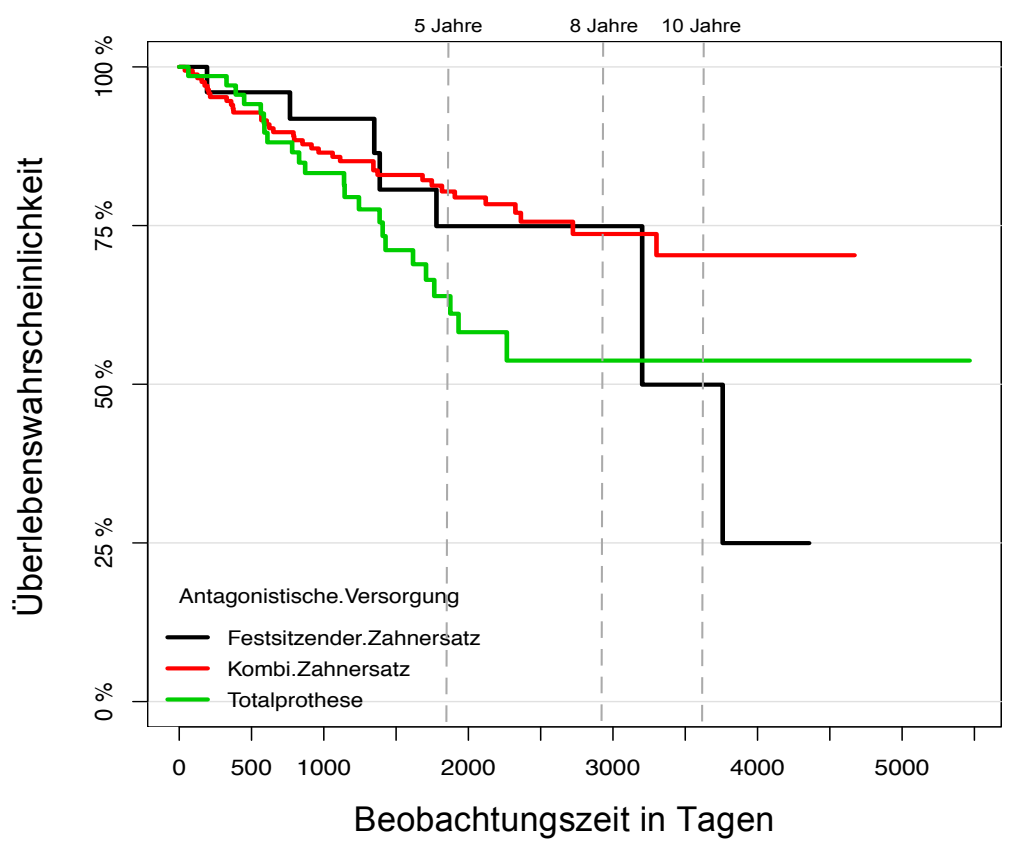

Abb. 21: Zeitabhängige Pfeilerüberlebenswahrscheinlichkeit (keine Fraktur) (nach Kaplan-Meier) der 263 Resilienzteleskopprothesen auf 538 Pfeilerzähnen in Abhängigkeit von der antagonistischen Versorgung 


\begin{tabular}{|c|c|c|c|c|}
\hline Variable & $\begin{array}{c}\text { Zeit } \\
\text { (Jahre) }\end{array}$ & $\begin{array}{c}\text { Festsitzender } \\
\text { Zahnersatz }\end{array}$ & $\begin{array}{c}\text { Kombi- } \\
\text { Zahnersatz }\end{array}$ & Totalprothesen \\
\hline \multirow{3}{*}{ Pfeilerfraktur } & 5 & $75 \%$ & $80 \%$ & $64 \%$ \\
\cline { 2 - 5 } & 8 & $75 \%$ & $74 \%$ & $54 \%$ \\
\cline { 2 - 5 } & 10 & $50 \%$ & $70 \%$ & $54 \%$ \\
\hline
\end{tabular}

Tab. 9: Zeitabhängige Pfeilerüberlebenswahrscheinlichkeit (keine Fraktur) der 263 Resilienzteleskopprothesen 


\subsubsection{Erfolgswahrscheinlichkeit bezogen auf Prothesenbasisfraktur}

Anhand der Kaplan-Meier-Analyse ließ sich eine prothesenbasisbezogene Erfolgswahrscheinlichkeit von $61 \%$ bei Resilienzteleskopprothesen mit antagonistischem festsitzenden Zahnersatz ohne Fraktur der Prothesenbasis nach 5 Jahren feststellen. Bei kombiniert festsitzend-abnehmbarem antagonistischen Zahnersatz konnten Erfolgsraten von 83\% und bei Totalprothesen von 80\% erzielt werden.

Nach einem Zeitraum von 8 Jahren betrug die Wahrscheinlichkeit bei festsitzendem Zahnersatz $46 \%$, bei kombiniert festsitzend-abnehmbarem Zahnersatz $75 \%$ und bei totalprothetischen Versorgungen 59\%.

Kombiniert festsitzend-abnehmbarer Zahnersatz als antagonistische Versorgung konnte - nach einem Beobachtungszeitraum von 10 Jahren - gegenüber Resilienzteleskopprothesen und einer nicht zu erwartenden Prothesenbasisfraktur die höchste Wahrscheinlichkeit von $66 \%$ zeigen. Gefolgt wurde dieses Ergebnis von festsitzendem Zahnersatz (46\%) und Totalprothesen (44\%). Die Darstellung ist in Abbildung 22 und Tabelle 10 ersichtlich.

Bei antagonistischem kombiniert festsitzend-abnehmbaren Zahnersatz konnte ein 0,33-fach geringeres Risiko für eine Basisfraktur in Relation zur Vergleichsgruppe (festsitzender antagonistischer Zahnersatz) festgestellt werden. Dieser Unterschied war statistisch signifikant $(p=0,0026)$. Eine Tendenz, jedoch keine Signifikanz, zeigte sich bei totalprothetischen antagonistischen Versorgungen relativ zur Vergleichsgruppe $(p=0,066)$. 


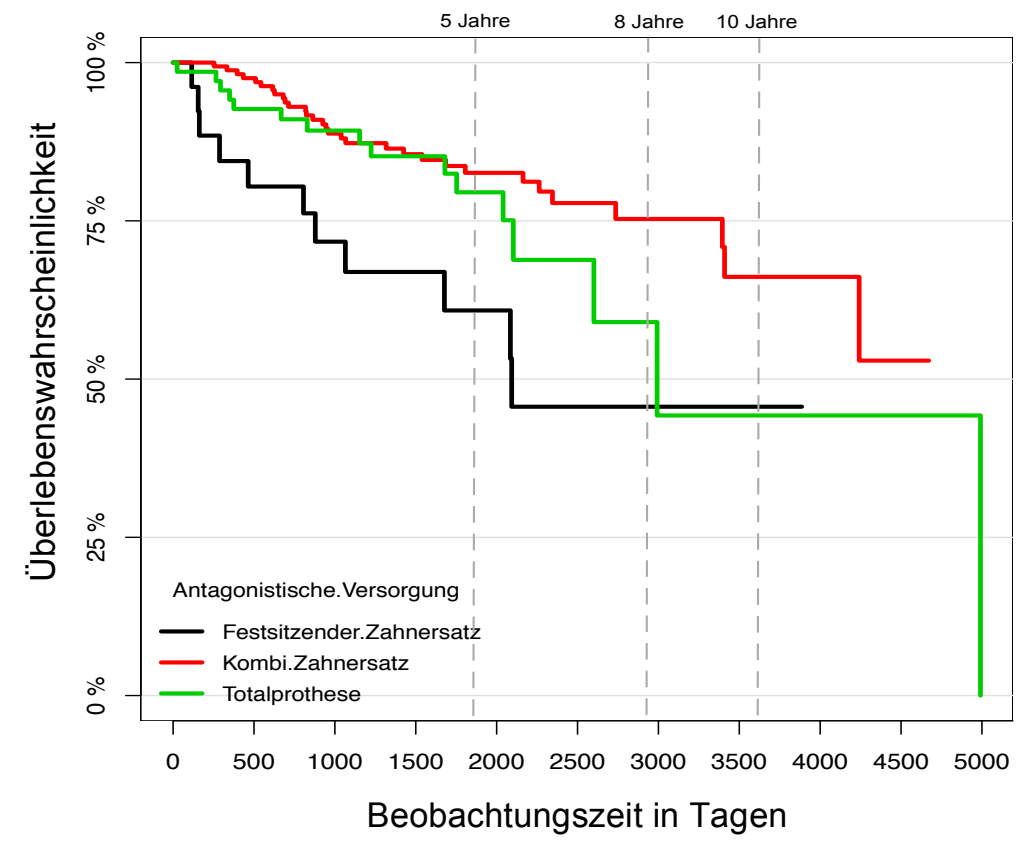

Abb. 22: Zeitabhängige Pfeilerüberlebenswahrscheinlichkeit (nach Kaplan-Meier) der 263 Resilienzteleskopprothesen auf 538 Pfeilerzähnen in Abhängigkeit von der antagonistischen Versorgung bezogen auf Prothesenbasisfraktur

\begin{tabular}{|c|c|c|c|c|}
\hline Variable & $\begin{array}{c}\text { Zeit } \\
\text { (Jahre) }\end{array}$ & $\begin{array}{c}\text { Festsitzender } \\
\text { Zahnersatz }\end{array}$ & $\begin{array}{c}\text { Kombi- } \\
\text { Zahnersatz }\end{array}$ & Totalprothesen \\
\hline \multirow{3}{*}{ Prothesenbasisfraktur } & 5 & $61 \%$ & $83 \%$ & $80 \%$ \\
\cline { 2 - 5 } & 8 & $46 \%$ & $75 \%$ & $59 \%$ \\
\cline { 2 - 5 } & 10 & $46 \%$ & $66 \%$ & $44 \%$ \\
\hline
\end{tabular}

Tab. 10: Zeitabhängige Erfolgswahrscheinlichkeit der 263 Resilienzteleskopprothesen bezüglich Prothesenbasisfraktur in Abhängigkeit von der antagonistischen Versorgung 


\section{Diskussion}

\subsection{Diskussion der Ergebnisse}

Die optimale Versorgung des stark reduzierten Restgebisses ist seit vielen Jahren von großer Bedeutung und war somit eine zentrale Fragestellung in klinischen Studien.

Die vorliegende Untersuchung zu Resilienzteleskopprothesen als prothetische Versorgungsmöglichkeit bei einem bis drei Restzähnen fokussierte sich auf die Langzeitbewährung und deren Komplikationsanfälligkeit. Leider ist die bisherige Datenlage zu dieser prothetischen Versorgungart rar, so dass sowohl starr verankerte teleskopierende Versorgungsmöglichkeiten als auch konische Doppelkronensysteme im Rahmen der Diskussion vergleichend herangezogen wurden.

Anhand der Ereigniszeitanalysen nach Kaplan-Meier wurde eine Überlebenswahrscheinlichkeit von Resilienzteleskopprothesen von $62 \%$ nach einer mittleren Beobachtungszeit von 5 Jahren, 38\% nach 8 Jahren und 28\% nach 10 Jahren ermittelt. Schwindling et al. gaben in ihrer Studie eine deutlich höhere Überlebenswahrscheinlichkeit für Resilienzteleskopprothesen von 90,4\% nach 5 Jahren an (Schwindling et al. 2014). Jedoch wurde in deren Studie nur eine geringe Fallzahl (34 Prothesen) untersucht, und die Überlebenswahrscheinlichkeit wurde nur bis zu einer Zeitspanne von 7 Jahren angegeben.

Rehmann et al. unterschritten 2007 in ihrer Longitudinalstudie über die Bewährung von Freiendteleskopen mit ausschließlicher Verankerung auf den Unterkiefereckzähnen die 50\%-Überlebenswahrscheinlichkeit der Prothesen erst nach 9,1 Jahren. Dabei sollten jedoch keine Rückschlüsse von einer beliebigen Zwei-PfeilerKonstellation auf die Eckzahnsituation gezogen werden (Rehmann et al. 2007). Eisenburger und Tschernitschek beschrieben in ihrer Studie zum Langzeiterfolg von Teleskopprothesen eine 50\%-Überlebenswahrscheinlichkeit nach 9,5 Jahren (Eisenburger und Tschernitschek 1998).

Des Weiteren konnte in der vorliegenden Untersuchung gezeigt werden, dass die Überlebenswahrscheinlichkeit mit zunehmender Anzahl der Pfeilerzähne im Laufe der untersuchten Beobachtungsintervalle steigt. 
Nach einer Beobachtungszeit von 5 Jahren betrug die Überlebenswahrscheinlichkeit für Resilienzteleskopprothesen mit einem Pfeilerzahn 39\%, mit zwei Pfeilerzähnen $63 \%$ und mit drei Pfeilerzähnen $80 \%$. Es konnte in der vorliegenden Studie ein signifikanter Unterschied zwischen Versorgungen mit einem bzw. zwei Pfeilerzähnen gegenüber Resilienzteleskopprothesen mit drei Pfeilerzähnen festgestellt werden $(p<0,001)$.

Schwindling et al. kamen zu dem Ergebnis, dass schwerwiegende Komplikationen bei Versorgungen mit Resilienzteleskopprothesen im Vergleich zu Teleskop- oder Konuskronen 2,2-fach häufiger auftraten (Schwindling et al. 2014). Zudem wurde ebenfalls ein negativer Effekt einer geringen Pfeileranzahl hinsichtlich der Überlebenszeit der Versorgungen beschrieben (Schwindling et al. 2014). Ähnliche Erkenntnisse konnten in einer Studie von Weber gewonnen werden. In dieser Untersuchung wiesen teleskopverankerte Teilprothesen mit drei Pfeilerzähnen eine signifikant höhere Überlebensdauer auf $(p=0,0464)$ als Prothesen mit zwei Pfeilern (Weber 2005). Ebenso kam Schüth zu dem Resultat, dass Teleskopprothesen mit $\geq 3$ Pfeilerzähnen eine längere Überlebenszeit vorweisen als Prothesen mit einem oder zwei Pfeilerzähnen (Schüth 1997). Wagner und Kern beschrieben in ihrer Studie über abnehmbare Teilprothesen eine Verbesserung des Therapieerfolges hinsichtlich einer höheren Pfeileranzahl sowie auch Rehmann et al. in ihrer Studie über Teilprothesen mit Teleskopkronen, welche ebenfalls einen Einfluss der Pfeileranzahl pro Prothese auf die Überlebenswahrscheinlichkeit der Pfeilerzähne feststellten (Wagner und Kern 2000; Rehmann et al. 2006).

Im Gegensatz dazu konnten Wenz et al. kein signifikant höheres Pfeilerverlustrisiko bei Teleskopprothesen mit starrer oder resilienter Lagerung, basierend auf dem Marburger Doppelkronensystem, mit drei oder weniger Restzähnen feststellen (Wenz et al. 2001). Stark und Schrenker ermittelten ebenfalls keinen negativen Einfluss einer geringeren Pfeilerzahl auf die Überlebensdauer von teleskopverankerten Prothesen (Stark und Schrenker 1998). In dieser Studie wurde allerdings nicht zwischen starr oder resilient verankerten Prothesen unterschieden. 
Die Überlebenswahrscheinlichkeit der Resilienzteleskopprothesen in Abhängigkeit von der jeweiligen Gegenkieferversorgung betrug nach den vorliegenden Ergebnissen bei festsitzendem Zahnersatz 66\%, bei kombiniert festsitzendabnehmbarem Zahnersatz $67 \%$ und bei totalprothetischer Versorgung $49 \%$ nach 5 Jahren; ein statistisch signifikanter Unterschied konnte jedoch nicht ermittelt werden.

Mock stellte in seiner Studie zur Langzeitbewährung von teleskopverankerten Prothesen einen signifikanten Einfluss der Gegenbezahnung hinsichtlich einiger Hygiene-Indizes fest. So zeigten sich beispielsweise bei Patienten mit einer natürlichen Gegenbezahnung oder rein gingival gelagertem Zahnersatz weniger Beläge auf der Prothesenunterseite und dem Pfeilerumfeld als bei Patienten mit einem parodontal-gingival gelagerten antagonistischen Zahnersatz (Mock 2005).

In der vorliegenden Studie konnte nach 5 Jahren die höchste Überlebenswahrscheinlichkeit der Prothesen bei antagonistischem kombiniert festsitzendabnehmbaren Zahnersatz festgestellt werden. Dieses Ergebnis wechselte jedoch im Laufe der Beobachtungszeit, was darauf schließen lässt, dass die Gegenkieferversorgung durchaus als möglicher zusätzlicher Einflussfaktor zur Verbesserung der Langzeitergebnisse der Resilienzteleskopprothesen gesehen werden kann, sich jedoch nicht konstant widerspiegelte.

Anhand der Ergebnisse konnte eine Erfolgswahrscheinlichkeit der Resilienzteleskopprothesen von 13\% nach 5 Jahren bestimmt werden. Versorgungen mit drei Pfeilerzähnen ergaben die beste Erfolgsrate von 20\%, gefolgt von zwei Pfeilerzähnen mit $11 \%$ und einem Pfeilerzahn mit $6 \%$. Außerdem ließ sich ein statistisch signifikanter Unterschied zwischen Resilienzteleskopprothesen mit zwei im Vergleich zu drei Pfeilerzähnen feststellen $(p=0,0353)$. Schlechtere Ergebnisse ermittelte Makowski in ihrer Studie über teleskopverankerte Prothesen. Dabei konnte nur eine Erfolgsrate von 8,6\% nach gleicher Beobachtungszeit festgestellt werden (Makowski 2010). Eine Ursache für diese Diskrepanz könnte die sofortige Wertung der technischen Komplikationen bei teleskopverankerten Prothesen nach dem Eingliederungsdatum sein. So stellten in dieser Studie Druckstellen die häufigste Komplikation dar $(71,7 \%)$, welche in der vorliegenden Studie erst ab einer Beobachtungszeit von 6 Monaten gewertet wurden. 
Schüth ermittelte in seiner Studie über die langfristige Bewährung von herausnehmbarem Zahnersatz eine durchschnittliche Zeit bis zur ersten Reparatur von 3,2 Jahren (Schüth 1997).

Vergleicht man die Erfolgswahrscheinlichkeit hinsichtlich der jeweiligen Gegenkieferversorgung konnte nach 5 Jahren bei antagonistischem festsitzenden Zahnersatz die beste Erfolgsrate von $25 \%$ festgestellt werden. Bei kombiniert festsitzend-abnehmbarem antagonistischen Zahnersatz wurde eine Erfolgswahrscheinlichkeit von $13 \%$ und $8 \%$ für antagonistische Totalprothesen nach dieser Zeit dokumentiert. Eine Steigerung der Erfolgswahrscheinlichkeit der Resilienzteleskopprothesen bei antagonistisch festsitzendem Zahnersatz kann somit vermutet, jedoch aufgrund mangelnder Untersuchungen in der Literatur nicht bestätigt werden.

Die untersuchten Resilienzteleskopprothesen wurden zum Großteil im Studentenkurs angefertigt und nur in Ausnahmefällen in der Assistentenbehandlung. Eine Verbesserung der Überlebenswahrscheinlichkeit der Versorgungen, unter der Behandlung von erfahrenen Zahnärzten, wäre zu erwarten und erklärt womöglich auch die im Vergleich zur Literatur schlechteren Erfolgs- und Überlebensraten.

Allerdings wurden die Studierenden gezielt und regelmäßig durch examinierte Zahnärzte betreut. So sollten eventuelle Fehler bei allen Behandlungsschritten in der Regel minimiert werden.

In der vorliegenden Studie wurde eine Pfeilerüberlebensrate von 55\% nach einer Beobachtungszeit von 5 Jahren, 34\% nach 8 Jahren und 30\% nach 10 Jahren dokumentiert. Versorgungen mit zwei Pfeilerzähnen ließen nach einer Beobachtungszeit von 5 Jahren ebenfalls eine Wahrscheinlichkeit von 55\% erkennen. Die Wahrscheinlichkeit für Resilienzteleskopprothesen mit einem Pfeilerzahn betrug jedoch nur 46\%, im Gegensatz zu Prothesen mit drei Pfeilerzähnen mit $62 \%$. Es wurde diesbezüglich ein statistisch signifikanter Unterschied zwischen Versorgungen mit einem und drei Pfeilerzähnen $(p=0,0207)$ ermittelt. Dieses Ergebnis unterlag somit deutlich der in vielen Untersuchungen aufgeführten Pfeilerüberlebenswahrscheinlichkeit von über $90 \%$ nach 5 Jahren und ca. $80 \%$ nach 8 bis 10 Jahren (Wenz und Kern 2007). 
Allerdings muss berücksichtigt werden, dass es sich bei der Mehrheit der Studien um teleskopierende, starre Doppelkronensysteme handelte und dadurch oft mehr als nur maximal 3 Pfeilerzähne mit in die prothetischen Versorgungen einbezogen wurden. Wenz et al. stellten bei ihrer Langzeitstudie über Resilienzteleskopprothesen ein geringeres Pfeilerverlustrisiko von 10\% nach 5 Jahren und 24\% nach 10 Jahren fest (Wenz et al. 2001). Dabei ist zu bemerken, dass alle Prothesen auf dem Marburger Doppelkronensystem basierten und die Anzahl der untersuchten Prothesen (insg. 125) wesentlich geringer war (Wenz et al. 2001).

Des Weiteren konnten Wenz et al. in ihrer Studie über Doppelkronen mit Spielpassung ein Risiko des Pfeilerverlustes bei starrer Lagerung ( $\geq 4$ Pfeilerzähne) von $4,8 \%$ und bei resilienter Lagerung ( $\leq 3$ Pfeilerzähne) von $8,7 \%$ nach 5 Jahren feststellen (Wenz et al. 1999).

Coca und Klimek stellten eine Überlebenswahrscheinlichkeit der Pfeilerzähne von $73 \%$ bei Cover-Denture-Prothesen mit Spielpassungsteleskopen im Unterkiefer nach 6,5 Jahren fest (Coca und Klimek 2002). Heners und Walther stellten bei Konuskronen eine statistisch signifikant schlechtere Prognose für den Pfeilerzahn, wenn die Prothese nur über 3 oder weniger Zähne verankert war (Heners und Walther 1990). Es kann somit ein Zusammenhang zwischen Pfeileranzahl sowie der Lagerungsart der Prothesen vermutet werden.

Eisenburger et al. stellten in ihrer retrospektiven Studie über Teleskopprothesen fest, dass sich keine besseren Überlebensraten unter Einbeziehung von mehr als 4 Pfeilerzähnen zeigten (Eisenburger et al. 2000). Außerdem konnten Szentpetery et al. in ihrer Studie über Friktionsteleskopkronen im stark reduzierten Restgebiss eine Pfeilerüberlebensrate von $80,6 \%$ nach einem Zeitraum von 5 Jahren verzeichnen (Szentpetery et al. 2012).

Eine mögliche Ursache für diese differierenden Ergebnisse könnte die schlechtere Pfeilerwertigkeit der jeweiligen in die Resilienzteleskopprothesen einbezogenen Zähne, vor allem im Vergleich zu Teleskopprothesen gewesen sein. In der vorliegenden Studie wiesen bereits 142 von den 271 ermittelten Zähnen einen Lockerungsgrad von 1 bis 3 auf. Die Pfeilerzähne waren zum Teil devital und wiesen einen erhöhten Substanzverlust auf. 
Aufgrund der aufgeführten Studien wurde deutlich, dass teleskopierende Versorgungen, im Vergleich zu resilient gelagerten Prothesen eine bessere Überlebenswahrscheinlichkeit der Pfeilerzähne aufwiesen. Die Ergebnisse der vorliegenden Studie sind hierzu konsistent, wobei die ermittelten Pfeilerüberlebenswahrscheinlichkeiten relativ zu vergleichbaren Studien unterlegen sind.

In der vorliegenden Studie konnte nach einer Beobachtungszeit von 5 Jahren ein frakturfreies Überleben der Pfeilerzähne mit einer Wahrscheinlichkeit von $76 \%$ festgestellt werden. Außerdem wurde ein statistisch signifikanter Unterschied zwischen einem und drei Pfeilerzähnen ermittelt $(p=0,0249)$. Rehmann et al. beschrieben in ihrer Studie über teleskopierende Teilprothesen die Fraktur als häufigste Ursache (47\%) für eine Pfeilerextraktion (Rehmann et al. 2006).

Als mögliche Ursache für das Ergebnis der vorliegenden Studie kann die alleinige Übertragung der Kaukräfte auf nur noch einen Pfeilerzahn und dessen Parodont gesehen werden. Rehmann et al. ermittelten bei vitalen Pfeilerzähnen eine signifikant höhere Überlebensrate $(p=0,015)$ gegenüber endodontisch behandelten Pfeilern (Rehmann et al. 2006, 2007). Ähnliche Erkenntnisse beschrieb Makowski in ihrer Studie über teleskopverankerte Prothesen. Dabei ließ sich eine signifikante Korrelation zwischen der Anzahl der verlorengegangenen Pfeilerzähne und der Anzahl der avitalen Pfeiler feststellen (Makowski 2010). Entgegen dieser Aussagen stellte sich Walther 1990, welcher in seiner Studie über Konuskronenkonstruktionen für den allein stehenden Pfeilerzahn kein erhöhtes Frakturrisiko als für den Pfeiler in umfangreicher abgestützten Konstruktionen feststellte. Er führte hingegen eine rigide Stufenpräparation als Risikofaktor an (Walther 1990).

Anhand der vorliegenden als auch anhand vieler angeführten Studien lässt sich schlussfolgern, dass prothetische Versorgungen, vor allem unter Einbeziehung vereinzelter Zähne, tendenziell einem höheren Risiko unterliegen.

Eine statistische Signifikanz ließ sich bei der antagonistischen Versorgung bezüglich einer Pfeilerfraktur nicht feststellen. Bei der antagonistischen Versorgung mit kombiniert festsitzend-abnehmbarem Zahnersatz konnte nach 5 Jahren die geringste Pfeilerfrakturrate festgestellt werden. Diese Wahrscheinlichkeit bestätigte sich im Laufe der Beobachtungszeit. 
Ein Grund für dieses Ergebnis könnte eine gute Harmonisierung der Resilienzteleskopprothesen mit der jeweiligen antagonistischen Prothese im Gegenkiefer sein. Eine zeitgleiche Versorgung beider Kiefer konnte während der Aufnahme der Daten vermehrt beobachtet werden und stellte somit eine gute Möglichkeit dar, beide Versorgungen sowohl funktionell als auch technisch einander anzugleichen.

In der vorliegenden Untersuchung konnte gezeigt werden, dass die Wahrscheinlichkeit für biologische Komplikationen bei Resilienzteleskopprothesen mit einem oder zwei Pfeilerzähnen höher ist als mit drei Restzähnen. Dieses Ergebnis konnte anhand der zwei bereits aufgeführten Komplikationen wie der Pfeilerverlust und die Frakturrate bestätigt werden.

Als ein möglicher Einflussfaktor kann die Position der Restzähne im jeweiligen Kiefer gesehen werden. Die Verankerung der Resilienzteleskopprothesen in der vorliegenden Studie erfolgte am häufigsten auf den unteren Eckzähnen (18\%). Pöggeler kam in seiner Studie über Cover-Denture-Prothesen zu der gleichen Erkenntnis (Pöggeler 1995). Mock ermittelte in seiner Untersuchung über teleskopverankerte Prothesen ebenfalls eine überwiegende Überkronung der Canini (Mock 2005). Bei der Mehrheit der Patienten verbleiben mit zunehmendem Alter vorwiegend im anterioren Bereich einige Zähne.

Die Auswirkungen der Verankerung auf unteren Eckzähnen auf die Überlebenswahrscheinlichkeit von Teleskopprothesen im teilbezahnten Gebiss untersuchten Rehmann et al. in ihrer Studie. Dabei konnte eine 90\%- (50\%-) Überlebenswahrscheinlichkeit der Pfeilerzähne nach 4 Jahren (12,9 Jahren) festgestellt werden (Rehmann et al. 2007).

Als mögliche Faktoren zur bevorzugten Einbeziehung der Eckzähne in die prothetische Versorgung könnten die günstige Morphologie sowie die ideale topographische Stellung im Zahnbogen angesehen werden. Sie stellen als die längsten Zähne im permanenten Gebiss (durchschnittlich $28 \mathrm{~mm}$ im Oberkiefer und $26 \mathrm{~mm}$ im Unterkiefer) eine optimale Verankerungsmöglichkeit dar (Lehmann et al. 2008). 
In der vorliegenden Studie konnte bei Resilienzteleskopprothesen, wie bereits beschrieben, eine deutlich schlechtere Überlebenswahrscheinlichkeit der Pfeilerzähne nach 5 Jahren ermittelt werden. Ein Grund für diese Diskrepanz könnte darin liegen, dass in der vorliegenden Arbeit alle Pfeilerzähne, unabhängig von deren Topographie, mit in die Versorgungen einbezogen wurden. Da beispielsweise Frontzähne eine ungünstigere Morphologie und damit Pfeilerwertigkeit aufweisen, ist die Art der Pfeilerzähne für die Gesamtprognose von Bedeutung. Des Weiteren konnte bei Rehmann von einer anderen Ausgangssituation ausgegangen werden, da es sich um Teleskopprothesen im teilbezahnten Gebiss handelte und somit ggf. zwischen potentiellen Pfeilern ausgewählt werden konnte.

In der vorliegenden Studie lag die biologische Erfolgsrate der Resilienzteleskopprothesen deutlich unter der technischen Komplikationsfreiheit nach einer Beobachtungszeit von 5 Jahren. Schlussfolgernd kann somit die Pfeilerwertigkeit als entscheidender Faktor für die Langzeitbewährung gesehen werden. Szentpetery et al. beschrieben eine signifikante Beeinflussung in ihrer Studie über Friktionsteleskopkronen im stark reduzierten Gebiss durch Geschlecht sowie Vitalität, Mobilität und Verteilung der Pfeilerzähne (Szentpetery et al. 2012).

Die günstigeren pfeileranzahlabhängigen Überlebensraten lassen sich möglicherweise u. a. auf eine verbesserte Retention bei Prothesen mit drei Restzähnen zurückführen. In der vorliegenden Studie ließen sich hinsichtlich der Pfeileranzahl signifikant schlechtere Überlebensraten zwischen Versorgungen mit einem von 79\% $(p=0,0471)$ und zwei Restzähnen von $81 \%(p=0,0277)$ gegenüber drei Pfeilerzähnen von $90 \%$ nach 5 Jahren feststellen.

In weiteren Studien wurde der Effekt der Retentionsverbesserung von Teilprothesen durch zusätzliche Pfeiler in Form von Implantaten untersucht. Krennmair et al. konnten in ihrer Studie über Implantate als Ergänzungsabutments, bei ImplantatZahn gestützten Teleskopprothesen im Oberkiefer gute Ergebnisse und geringe Komplikationen bestätigen (Krennmair et al. 2007). Durch die zusätzlichen implantologischen Pfeiler kann die auf die natürlichen Restzähne einwirkende Belastung reduziert werden (Sato et al. 2013; Mijiritsky et al. 2005; Levin 2008). 
Hofmann und Ludwig beurteilten die Haltwirkung bei Resilienzteleskopprothesen über einen Beobachtungszeitraum von 2-8 Jahren positiv (Hofmann und Ludwig 1973). Ein Faktor für diese Diskrepanz konnte die jeweils individuelle Position der Restzähne sein. Außerdem wurden in der vorliegenden Studie vermehrt Prothesen mit einem oder zwei Restzähnen eingeschlossen. Dies könnte ebenfalls ein Grund für die schlechteren Ergebnisse hinsichtlich der Retention sein. Zum anderen lässt sich die längere Überlebenswahrscheinlichkeit bei Resilienzteleskopprothesen mit drei Pfeilerzähnen auf die geringere mechanische Belastung der einzelnen Pfeiler zurückführen. Durch die gleichmäßigere Verteilung der Kau- und Scherkräfte kann beispielsweise das Auftreten von Parodontopathien reduziert werden.

Bereits Körber et al. beschrieben die Abhängigkeit der Zahnlockerung von der Pfeileranzahl (Körber et al. 1978). Diese Studie basierte auf einer Untersuchung von Friktionsteleskopen.

Hofmann und Ludwig kamen bei ihrer Untersuchung von 163 Resilienzteleskopen zu dem Ergebnis, dass zunächst die Tendenz der Zahnbeweglichkeit innerhalb der ersten 3 Jahre abnahm, sich jedoch bei fortschreitender Tragedauer erhöhte (Hofmann und Ludwig 1973). Die Untersuchung des Lockerungsgrades über einen längeren Zeitraum konnte in der vorliegenden Studie aufgrund unzureichender Dokumentation nur vereinzelt erfolgen und bot deshalb keine ausreichende Untersuchungs- und Diskussionsgrundlage.

Rehmann et al. nannten einzig die Vitalität der Pfeilerzähne als signifikanten Einflussfaktor zur Sicherung eines langfristigen Erfolges der Pfeilerzähne (Rehmann et al. 2007).

Als eine weitere Ansatzmöglichkeit zur Steigerung des Langzeiterfolges kann die technische Gestaltungsmöglichkeit der Resilienzteleskopprothesen gesehen werden. Coca et al. beschrieben in ihrer Studie 106 resilient gelagerte Deckprothesen (mit funktioneller Randgestaltung nach dem "Cover-Denture“ Prinzip), während hingegen Wenz et al. ein perioprothetisches Design, mit Freihaltung des marginalen Parodonts, eine brückenzwischengliedartige Gestaltung der pfeilerzahnnahen Prothesensättel sowie den Verzicht auf den großen Verbinder als auch einen TKSnap als zusätzliches Halteelement wählten (Coca et al. 2000; Pöggeler 1995; Wenz et al. 1999, 2001). 
Durch diese Gestaltung konnte nach einer Beobachtungszeit von 7 Jahren ein deutlich besseres Ergebnis für das perioprothetische Design erzielt werden. In der vorliegenden Studie wurden die Prothesen ebenfalls nach dem "Cover-Denture" Prinzip hergestellt. Eine parodontalfreundlichere Gestaltung sollte somit in jedem Fall geprüft werden.

In der vorliegenden Studie wurde außerdem anhand der Kaplan-Meier-Analyse ein statistisch signifikanter Unterschied bei Resilienzteleskopprothesen in Abhängigkeit ihrer jeweiligen antagonistischen Versorgung in Hinsicht auf eine Prothesenbasisfraktur festgestellt. Ein geringeres Risiko für eine Fraktur der Prothesenbasis bei resilient gelagerten Versorgungen stellte kombiniert festsitzendabnehmbarer Zahnersatz $(p=0,0026)$ im Vergleich zu festsitzendem Zahnersatz dar.

Nach 5 Jahren ließ sich mit einer Wahrscheinlichkeit von $61 \%$ bei Resilienzteleskopprothesen in Abhängigkeit von festsitzendem Zahnersatz keine Fraktur der Prothesenbasis feststellen. Totalprothesen erzielten eine Wahrscheinlichkeit von $80 \%$ und kombiniert festsitzend-abnehmbarer Zahnersatz die geringste Wahrscheinlichkeit mit 83\%.

Ein Grund für das erhöhte Risiko in Abhängigkeit von festsitzendem Zahnersatz für eine Prothesenbasisfraktur könnte die ungleichmäßige Kräfteverteilung bzw. Belastung gewesen sein. Außerdem ist der Spielraum für die Resilienzteleskopprothese sehr begrenzt, da sich festsitzende Restaurationen nicht wie beispielsweise teleskopierende Prothesen bei Freiendsituationen in die Schleimhaut einlagern. Totalprothesen, aber auch kombiniert festsitzend-abnehmbare Prothesen, können somit die Voraussetzungen für ein weiches Widerlager besser erfüllen.

Mock stellte in seiner Untersuchung einen signifikanten Einfluss der Gegenbezahnung hinsichtlich des Lockerungsgrades der Pfeilerzähne fest (Mock 2005). Eine „physiologische Beweglichkeit“ ergab sich bei natürlicher Gegenbezahnung und eine „,ühlbare Beweglichkeit" zeigte sich bei rein gingival gelagertem Zahnersatz oder parodontal-gingivaler Gegenbezahnung (Mock 2005).

In der vorliegenden Studie wurden ebenfalls biologische Komplikationen der Pfeilerzähne im Zusammenspiel mit der antagonistische Versorgung untersucht. Es konnte keine statistische Signifikanz festgestellt werden. 
Es ließen sich bei Resilienzteleskopprothesen in Abhängigkeit von festsitzendem Zahnersatz mit einer Wahrscheinlichkeit von $68 \%$, bei kombiniert festsitzendabnehmbarem Zahnersatz von $43 \%$ und bei totalprothetischen Versorgungen von $37 \%$ keine biologischen Komplikationen nach 5 Jahren feststellen. Ein Grund für dieses Ergebnis könnte die gleichmäßigere, physiologische Belastung der Pfeilerzähne bei festsitzendem bzw. natürlichem Zahnersatz sein.

Auch ein möglicher Zusammenhang zwischen einer schlechteren Mundhygiene und erhöhtem Auftreten von Sekundärkaries in der Vergangenheit bei Vorhandensein einer Totalprothese (81\% Komplikationsfreiheit) im Vergleich zu festsitzendem Zahnersatz (89\% Komplikationsfreiheit) könnte vermutet werden.

\subsection{Diskussion der Methodik}

Im Rahmen der vorliegenden Studie konnte aufgrund der hohen Anzahl von 263 Resilienzteleskopprothesen sowie 538 Pfeilerzähnen eine umfangreiche und repräsentative Analyse durchgeführt werden. Die aufgeführte Fallzahl ist zu vergleichbaren bisher veröffentlichten Studien zu dieser prothetischen Versorgungsart relativ hoch. Hofmann und Ludwig untersuchten 78 Resilienzteleskopprothesen (Hofmann und Ludwig 1973). Coca und Klimek schlossen 236 Doppelkronen in ihrer Studie über Cover-Dentures mit Spielpassungsteleskopen ein (Coca und Klimek 2002). Allerdings bezog sich diese Studie nur auf Restzähne im Unterkiefer. Schwindling et al. bezogen 117 Prothesen in ihre Studie ein, wobei nur 34 Versorgungen aus resilient getragenen Doppelkronen bestanden (Schwindling et al. 2014). Erwähnt werden sollte an dieser Stelle nochmals, dass die bisherige Datenlage zu dieser prothetischen Versorgungart rar ist und ihre Anwendung nur in einigen Ländern positiven Zuspruch erlangte.

Die vorliegende Studie konnte eine mittlere klinische Beobachtungsdauer von 64,5 Monaten (5,4 Jahren) verzeichnen. Dabei ergaben sich individuelle Beobachtungszeiträume von 3 bis 179 Monaten, wobei sich die Zeiträume unterhalb der Mindestbeobachtungsdauer von 24 Monaten nur auf frühzeitig verloren gegangene Versorgungen bezogen. Es wurden Ereigniszeitanalysen nach Kaplan-Meier sowie eine multivariate Cox-Regression im Intervall von 3, 5, 8 und 10 Jahren durchgeführt. 
Vergleicht man diese Beobachtungszeiträume mit anderen Studien lassen sich ähnliche Werte finden. So gaben Wenzel et al. in ihrer Untersuchung für die aufgeführten Resilienzteleskopprothesen Beobachtungsintervalle von 5 und 10 Jahren an (Wenz et al. 2001). Heners und Walther wählten in ihrer klinischen Langzeitstudie einen Beobachtungszeitraum von 6 Jahren aus (Heners und Walther 1988). Hofmann und Ludwig beschrieben eine ermittelte Tragedauer von 2 bis 8 Jahren (Hofmann und Ludwig 1973).

Die vorliegende retrospektive Studie basierte auf einer Aktenanalyse der in der Poliklinik für Zahnärztliche Prothetik der Medizinischen Fakultät der Universität Göttingen behandelten Patienten.

Aufgrund des zum Teil unregelmäßigen Erscheinens der Patienten zu den empfohlenen Recall-Terminen, gestaltete sich die ausführliche Dokumentation und Nachvollziehbarkeit der aufgetretenen Komplikationen teilweise schwierig und lückenhaft. Des Weiteren war die ausführliche Erfassung der durchgeführten Behandlungen sehr von der Dokumentation des jeweiligen behandelnden Zahnarztes abhängig.

Die strukturierte, alleinige Dokumentation von nur einem behandelnden Zahnarzt wäre vorteilhaft gewesen, dies ist bei retrospektiven Studien in der Regel jedoch nicht zu erwarten. Eine strukturierte klinische Nachuntersuchung erfolgte in dieser Studie ebenfalls nicht. Auch dies hätte die Validität der Daten ggf. gesteigert, hätte aber einen ungleich höheren Aufwand mit unvorhersehbarer Recall-Quote im Sinne der Verfügbarkeit der Patienten bedeutet. So endete die Erfassung mit dem zuletzt vermerkten Recall-Termin in der jeweiligen Patientenakte.

Das retrospektive Studiendesign gestaltete sich sowohl aus ethischer als auch aus finanzieller Sicht als vorteilhaft. Die Untersuchung erfolgte hierbei an bereits vorhandenen Datenmaterialien. Der Großteil aller bisher veröffentlichten Studien zu Resilienzteleskopprothesen und teleskopverankerten Versorgungen bediente sich dieser Methode. Demgegenüber standen prospektive Longitudinalstudien, wie es beispielsweise durch Mock in seiner Untersuchung über die Bewährung von teleskopverankerten Prothesen gezeigt wurde (Mock 2005). Dabei wurden die wichtigsten Parameter vor Anfall des Datenmaterials definiert. 
Dieses konnte somit anschließend genau auf die Anforderungen der Studie zugeschnitten werden. Das Kriterium konnte in der vorliegenden Studie als negativ bewertet werden. Aufgrund teilweise fehlender Daten konnte nicht immer alle Anforderungen der Studie erfüllt werden. Die Ursachenforschung konnte sich somit nur auf das gefundene Datenmaterial beschränken. Als deutlicher Vorteil konnten die schnellere Durchführbarkeit und der stark reduzierte administrative Aufwand sowie die Kosten im Vergleich zu einem prospektiven Studiendesign gesehen werden.

Insgesamt ließen sich wesentlich weniger und vermehrt ältere Studien bzw. Nachuntersuchungen zu Resilienzteleskopprothesen als zu teleskopverankerten Versorgungen finden. Dies könnte zum einen an der festen Vorgabe einer begrenzten Anzahl der Restzähne sowie der damit verbundenen erschwerten Patienten-Akquirierung liegen. Zum anderen handelte es sich um eine spezielle Doppelkronenart mit resilienter Lagerung, welche in Deutschland nicht überall verbreitet ist.

1/3 der nachuntersuchten Resilienzteleskopprothesen konnten in die vorliegende Studie und die anschließende Auswertung nicht mit einbezogen werden. Anhand der Aktendokumentation wurde bei einigen Patienten, nach dem Eingliederungsdatum der Prothese, keine erneute Vorstellung in der Poliklinik für Zahnärztliche Prothetik ersichtlich. Somit konnte diese Patientengruppe den vorgegebenen Beobachtungszeitraum von mindestens 24 Monaten nicht erfüllen. Eine Folgeuntersuchung hätte bei diesen Patienten als sinnvoll angesehen werden können. Dies sollte aus genannten Gründen jedoch nicht erfolgen.

Des Weiteren konnte bei einigen Patienten nur das Eingliederungs- und Verlustdatum festgestellt werden, jedoch ohne erfolgte Recall-Behandlungen bzw. fortlaufender Dokumentation. Diese Patienten konnten ebenfalls keine repräsentativen Ergebnisse liefern. Darüber hinaus ließ sich in einigen Patientenakten kein ersichtlicher Grund für den Verlust oder die Neuanfertigung der Resillienzteleskopprothesen finden. Diese Patientengruppe wurde kritisch analysiert, da dies teilweise der unzureichenden Dokumentation des jeweilig behandelnden Zahnarztes geschuldet war. Die Einbeziehung in die vorliegende Studie wurde somit für jeden Fall individuell und kritisch entschieden. 
Des Weiteren muss die Auswahl der untersuchten Parameter kritisch betrachtet werden. Pöggeler führte getrennte Überlebenszeitanalysen nach Kaplan-Meier im Ober- und Unterkiefer durch (Pöggeler 1995). Diese Analyse hätte durchaus als eine weitere mögliche Untersuchung in Erwägung gezogen werden können. Stark und Schrenker untersuchten in ihrer klinischen Langzeitstudie über teleskopverankerte Prothesen den Entzündungs- und Lockerungsgrad der Pfeilerzähne bei Männern und Frauen (Stark und Schrenker 1998). Diese Parameter hätten in der vorliegenden Studie ebenfalls als sinnvoll angesehen werden können.

Behr et al. verglichen Teleskop- und Konuskronen getragene Doppelkronensysteme hinsichtlich der technischen Komplikationsraten miteinander (Behr et al. 2000). Schwindling et al. führten eine vergleichende Studie von Teleskop,- Konus- und resilient getragenen Doppelkronenprothesen hinsichtlich der Überlebens- sowie Komplikationsraten durch (Schwindling et al. 2014). Eine Analyse in dieser Form wäre in der Poliklinik für Zahnärztliche Prothetik der Medizinischen Fakultät der Universität Göttingen zum damaligen Zeitpunkt aufgrund fehlender konischer Doppelkronenprothesen nicht möglich gewesen. Kritisch betrachtet werden muss jedoch in der Untersuchung von Schwindling die relativ geringe Fallzahl, da dies die Allgemeingültigkeit der Ergebnisse begrenzt.

Ziel der vorliegenden Studie war es, anhand der hohen Prothesenanzahl sowie der fokussierten Analyse auf eine Versorgungsart eine kritische und ausführliche Bewertung bezüglich möglicher Komplikationen und den zu erwartenden Langzeitergebnissen vorzunehmen. Aus diesem Grund wurden gezielt alle möglichen biologischen und technischen Komplikationen aufgenommen und ausgewertet. Ein ähnliches Vorgehen konnte bei Makowski in ihrer Studie über die häufigsten Reparaturen bei teleskopverankerten Prothesen identifiziert werden (Makowski 2010). 


\section{Schlussfolgerung}

Die in der vorliegenden Studie untersuchten Resilienzteleskopprothesen konnten den durchschnittlichen Anforderungen an Zahnersatz bezüglich einer guten Langzeitbewährung und geringen Komplikationsraten nicht gerecht werden.

Anhand der Kaplan-Meier-Analyse konnte eine Überlebenswahrscheinlichkeit ermittelt werden, welche nicht mit den Ergebnissen bisher durchgeführter Studien zu Cover-Denture-Prothesen einhergeht. Eine Verbesserung der Überlebenswahrscheinlichkeit mit zunehmender Anzahl der Pfeilerzähne im Laufe der untersuchten Beobachtungsintervalle wurde wiederum in vielen anderen Studien bestätigt. Schwindling et al. beschrieben in ihrer Studie über Doppelkronengetragene Prothesen einen negativen Effekt einer geringen Pfeileranzahl hinsichtlich der Überlebenszeit der Resilienzteleskopprothesen (Schwindling et al. 2014). Rehmann et al. stellten ebenfalls in ihrer Studie über Teilprothesen mit Teleskopkronen einen Einfluss der Pfeileranzahl pro Prothese auf die Überlebenswahrscheinlichkeit der Pfeilerzähne fest (Rehmann et al. 2006).

Außerdem konnte in der vorliegenden Studie festgestellt werden, dass sich auch hinsichtlich der Erfolgsraten und biologischen sowie technischen Komplikationen ein Zusammenhang zur Anzahl der Pfeilerzähne zeigte. Dies bezog sich zum einen auf die Pfeilerverlust- und Frakturraten und zum anderen auf den Retentionsverlust. Eine vergleichbare Erkenntnis wurde bei bisher durchgeführten Studien zu Resilienzteleskopprothesen nicht beschrieben, bei anderen Doppelkronenstudien jedoch schon.

Aufgrund der drastischen Komplikationen sowie Verlustraten kann die Resilienzteleskopprothese als kombiniert festsitzend-abnehmbarer Zahnersatz im stark reduzierten Restgebiss bei einem bis drei Restzähnen nicht mehr empfohlen werden. Es ließen sich jedoch anhand der vorliegenden und bereits durchgeführten Studien Faktoren feststellen, welche sich positiv auf die Überlebenswahrscheinlichkeit der Pfeilerzähne auswirkten. Somit konnten durch eine günstige Verteilung und höhere Anzahl der Pfeilerzähne komplikationsreduzierende Voraussetzungen geschaffen werden. Außerdem lassen sich ein funktionierendes Recall-System sowie eine parodontalhygienische Gestaltung des Prothesenkörpers als positive Einflussfaktoren vermuten. 
Als Therapiealternative können anhand der durchgeführten Studie sowie der heutigen Erkenntnis teleskopierende Doppelkronenversorgungen ohne Resilienzspielraum gesehen werden. Dabei ist eine strenge Pfeilerselektion zur Sicherung eines höheren Langzeiterfolges ebenfalls von großer Bedeutung. 


\section{Zusammenfassung}

Zur Beurteilung der langfristigen Bewährung von Resilienzteleskopprothesen wurden 221 Patientenakten mit 263 Versorgungen auf insgesamt 538 Pfeilerzähnen, welche in der Poliklinik für Zahnärztliche Prothetik der Medizinischen Fakultät der Universität Göttingen versorgt wurden, untersucht. Es ergaben sich individuelle Beobachtungszeiträume von 3 bis 179 Monaten mit einer mittleren klinischen Beobachtungsdauer von 64,5 Monaten (5,4 Jahren).

Es konnte anhand der Kaplan-Meier-Analyse insgesamt eine Überlebenswahrscheinlichkeit von $62 \%$ nach 5 Jahren, $38 \%$ nach 8 Jahren und $28 \%$ nach 10 Jahren festgestellt werden. Außerdem wurde ermittelt, dass die Überlebenswahrscheinlichkeit mit zunehmender Anzahl der Pfeilerzähne im Laufe der untersuchten Beobachtungsintervalle steigt. Die Erfolgswahrscheinlichkeit der untersuchten 263 Resilienzteleskopprothesen betrug nach 5 Jahren 13\%. Nach einem Zeitraum von 8 Jahren wird nur noch eine Erfolgswahrscheinlichkeit von 3\% und nach 10 Jahren von 1\% ersichtlich. Eine Pfeilerüberlebensrate von 55\% ergab sich nach einem Beobachtungszeitraum von 5 Jahren. Diese ging nach einem Zeitraum von 8 Jahren auf 34\% und nach 10 Jahren schließlich auf $30 \%$ zurück.

Es konnten signifikant höhere Komplikationsraten wie Pfeilerfrakturen, Pfeilerverluste oder Retentionsverluste bei Versorgungen mit einem oder zwei Pfeilerzähnen im Vergleich zu Resilienzteleskopprothesen mit drei Restzähnen festgestellt werden. Eine statistische Signifikanz konnte ebenfalls bei der Variablen Prothesenbasisfraktur in Abhängigkeit von der antagonistischen Versorgung festgestellt werden. Dabei konnte bei kombiniert festsitzend-abnehmbarem Zahnersatz ein geringeres Risiko für eine Fraktur feststellt werden als bei festsitzendem Zahnersatz. Eine Empfehlung der Resilienzteleskopprothesen als kombiniert festsitzendabnehmbaren Zahnersatz im stark reduzierten Restgebiss bei einem bis drei Restzähnen kann aufgrund der drastischen Komplikationen und Verlustraten nicht mehr ausgesprochen werden. Eine gute Langzeitbewährung war bei dieser Versorgungsart nicht mehr ersichtlich. Jedoch stellten eine günstige Verteilung und höhere Anzahl der Pfeilerzähne sowie regelmäßige Kontrolluntersuchungen Ansatzmöglichkeiten dar, die Langzeitperspektive von Resilienzteleskopprothesen zu verbessern. 


\section{Anhänge}

\begin{tabular}{|c|c|c|c|}
\hline Variable & Zeit [Tage] & Wahrscheinlichkeit & Konfidenzintervall \\
\hline \multirow[t]{4}{*}{ Überleben } & 1095 & 0.8 & {$[0.74 ; 0.85]$} \\
\hline & 1825 & 0.62 & {$[0.55 ; 0.69]$} \\
\hline & 2920 & 0.38 & {$[0.3 ; 0.45]$} \\
\hline & 3650 & 0.28 & {$[0.2 ; 0.36]$} \\
\hline \multirow[t]{4}{*}{ Erfolg } & 1095 & 0.29 & {$[0.23 ; 0.35]$} \\
\hline & 1825 & 0.13 & {$[0.09 ; 0.17]$} \\
\hline & 2920 & 0.03 & {$[0.01 ; 0.05]$} \\
\hline & 3650 & 0.01 & {$[0 ; 0.03]$} \\
\hline \multirow{4}{*}{$\begin{array}{l}\text { Erfolg } \\
\text { Techn.Komplikation }\end{array}$} & 1095 & 0.73 & {$[0.67 ; 0.79]$} \\
\hline & 1825 & 0.63 & {$[0.57 ; 0.7]$} \\
\hline & 2920 & 0.53 & {$[0.45 ; 0.62]$} \\
\hline & 3650 & 0.47 & {$[0.36 ; 0.59]$} \\
\hline \multirow{4}{*}{$\begin{array}{l}\text { Erfolg } \\
\text { Biol. Komplikation }\end{array}$} & 1095 & 0.54 & {$[0.48 ; 0.6]$} \\
\hline & 1825 & 0.44 & {$[0.38 ; 0.51]$} \\
\hline & 2920 & 0.37 & {$[0.31 ; 0.44]$} \\
\hline & 3650 & 0.37 & {$[0.31 ; 0.44]$} \\
\hline \multirow{4}{*}{$\begin{array}{l}\text { Erfolg } \\
\text { Pfeilerverlust }\end{array}$} & 1095 & 0.75 & {$[0.7 ; 0.81]$} \\
\hline & 1825 & 0.55 & {$[0.48 ; 0.62]$} \\
\hline & 2920 & 0.34 & {$[0.27 ; 0.42]$} \\
\hline & 3650 & 0.3 & {$[0.22 ; 0.38]$} \\
\hline \multirow{4}{*}{$\begin{array}{l}\text { Erfolg } \\
\text { Sekundärkaries }\end{array}$} & 1095 & 0.9 & {$[0.86 ; 0.94]$} \\
\hline & 1825 & 0.85 & {$[0.8 ; 0.89]$} \\
\hline & 2920 & 0.78 & {$[0.71 ; 0.85]$} \\
\hline & 3650 & 0.71 & {$[0.61 ; 0.81]$} \\
\hline \multirow{4}{*}{$\begin{array}{l}\text { Erfolg } \\
\text { Vitalitätsverlust }\end{array}$} & 1095 & 0.88 & {$[0.84 ; 0.92]$} \\
\hline & 1825 & 0.82 & {$[0.77 ; 0.87]$} \\
\hline & 2920 & 0.73 & {$[0.66 ; 0.8]$} \\
\hline & 3650 & 0.73 & {$[0.66 ; 0.8]$} \\
\hline \multirow{4}{*}{$\begin{array}{l}\text { Erfolg } \\
\text { Pfeilerfraktur }\end{array}$} & 1095 & 0.86 & {$[0.81 ; 0.9]$} \\
\hline & 1825 & 0.76 & {$[0.7 ; 0.82]$} \\
\hline & 2920 & 0.69 & {$[0.62 ; 0.76]$} \\
\hline & 3650 & 0.64 & {$[0.55 ; 0.73]$} \\
\hline \multirow{4}{*}{\begin{tabular}{|l} 
Erfolg \\
Retentionsverlust
\end{tabular}} & 1095 & 0.86 & {$[0.82 ; 0.91]$} \\
\hline & 1825 & 0.83 & {$[0.79 ; 0.88]$} \\
\hline & 2920 & 0.79 & {$[0.73 ; 0.85]$} \\
\hline & 3650 & 0.76 & {$[0.67 ; 0.84]$} \\
\hline \multirow{4}{*}{$\begin{array}{l}\text { Erfolg } \\
\text { Prothesenzahnfraktur }\end{array}$} & 1095 & 0.9 & {$[0.86 ; 0.94]$} \\
\hline & 1825 & 0.86 & {$[0.81 ; 0.9]$} \\
\hline & 2920 & 0.83 & {$[0.78 ; 0.88]$} \\
\hline & 3650 & 0.79 & {$[0.72 ; 0.86]$} \\
\hline \multirow{4}{*}{$\begin{array}{l}\text { Erfolg } \\
\text { Prothesenbasisfraktur }\end{array}$} & 1095 & 0.86 & {$[0.81 ; 0.9]$} \\
\hline & 1825 & 0.8 & {$[0.74 ; 0.85]$} \\
\hline & 2920 & 0.69 & {$[0.61 ; 0.77]$} \\
\hline & 3650 & 0.6 & {$[0.48 ; 0.71]$} \\
\hline
\end{tabular}

Tab. 11: Ereignis-Zeitanalysen (Kaplan-Meier) nach jeweils 3, 5, 8 und 10 Jahren mit 95\%-Konfidenzintervall 


\begin{tabular}{|c|c|c|c|c|}
\hline Variable & Zeit [Tage] & Pfeileranzahl 1 & Pfeileranzahl 2 & Pfeileranzahl 3 \\
\hline \multirow[t]{4}{*}{ Überleben } & 1095 & $0.64[0.53 ; 0.75]$ & $0.78[0.7 ; 0.86]$ & $0.95[0.91 ; 1]$ \\
\hline & 1825 & $0.39[0.27 ; 0.51]$ & $0.63[0.53 ; 0.73]$ & $0.8[0.72 ; 0.89]$ \\
\hline & 2920 & $0.16[0.07 ; 0.25]$ & $0.27[0.16 ; 0.38]$ & $0.7[0.6 ; 0.81]$ \\
\hline & 3650 & $0.16[0.07 ; 0.25]$ & $0.2[0.09 ; 0.3]$ & $0.48[0.31 ; 0.65]$ \\
\hline \multirow[t]{4}{*}{ Erfolg } & 1095 & $0.24[0.14 ; 0.34]$ & $0.26[0.18 ; 0.35]$ & $0.36[0.26 ; 0.47]$ \\
\hline & 1825 & $0.06[0.01 ; 0.11]$ & $0.11[0.05 ; 0.18]$ & $0.2[0.11 ; 0.28]$ \\
\hline & 2920 & $\mathrm{NA}[\mathrm{NA} ; \mathrm{NA}]$ & $0.01[0 ; 0.03]$ & $0.07[0.02 ; 0.12]$ \\
\hline & 3650 & NA [NA;NA] & $\mathrm{NA}[\mathrm{NA} ; \mathrm{NA}]$ & $0.03[0 ; 0.07]$ \\
\hline \multirow{4}{*}{$\begin{array}{l}\text { Erfolg } \\
\text { Techn. Komplikation }\end{array}$} & 1095 & $0.68[0.56 ; 0.79]$ & $0.71[0.62 ; 0.81]$ & $0.79[0.7 ; 0.87]$ \\
\hline & 1825 & $0.49[0.35 ; 0.64]$ & $0.62[0.52 ; 0.73]$ & $0.72[0.62 ; 0.81]$ \\
\hline & 2920 & $0.42[0.25 ; 0.59]$ & $0.59[0.47 ; 0.71]$ & $0.57[0.44 ; 0.7]$ \\
\hline & 3650 & $0.42[0.25 ; 0.59]$ & $0.49[0.31 ; 0.68]$ & $0.51[0.35 ; 0.67]$ \\
\hline \multirow{4}{*}{$\begin{array}{l}\text { Erfolg } \\
\text { Biol. Komplikation }\end{array}$} & 1095 & $0.5[0.39 ; 0.62]$ & $0.54[0.44 ; 0.64]$ & $0.57[0.47 ; 0.67]$ \\
\hline & 1825 & $0.4[0.29 ; 0.52]$ & $0.45[0.34 ; 0.55]$ & $0.47[0.37 ; 0.57]$ \\
\hline & 2920 & $0.34[0.22 ; 0.46]$ & $0.34[0.22 ; 0.45]$ & $0.43[0.33 ; 0.53]$ \\
\hline & 3650 & $0.34[0.22 ; 0.46]$ & $0.34[0.22 ; 0.45]$ & $0.43[0.33 ; 0.53]$ \\
\hline \multirow{4}{*}{$\begin{array}{l}\text { Erfolg } \\
\text { Pfeilerverlust }\end{array}$} & 1095 & $0.66[0.55 ; 0.77]$ & $0.74[0.66 ; 0.83]$ & $0.83[0.76 ; 0.91]$ \\
\hline & 1825 & $0.46[0.33 ; 0.59]$ & $0.55[0.45 ; 0.66]$ & $0.62[0.51 ; 0.73]$ \\
\hline & 2920 & $0.23[0.11 ; 0.34]$ & $0.25[0.14 ; 0.36]$ & $0.53[0.41 ; 0.65]$ \\
\hline & 3650 & $0.23[0.11 ; 0.34]$ & $0.25[0.14 ; 0.36]$ & $0.39[0.25 ; 0.54]$ \\
\hline \multirow{4}{*}{$\begin{array}{l}\text { Erfolg } \\
\text { Sekundärkaries }\end{array}$} & 1095 & $0.92[0.85 ; 0.99]$ & $0.91[0.85 ; 0.97]$ & $0.88[0.81 ; 0.95]$ \\
\hline & 1825 & $0.86[0.76 ; 0.96]$ & $0.84[0.76 ; 0.92]$ & $0.84[0.76 ; 0.92]$ \\
\hline & 2920 & $0.77[0.6 ; 0.94]$ & $0.77[0.66 ; 0.88]$ & $0.79[0.69 ; 0.89]$ \\
\hline & 3650 & $0.64[0.4 ; 0.88]$ & $0.66[0.46 ; 0.86]$ & $0.76[0.64 ; 0.88]$ \\
\hline \multirow{4}{*}{$\begin{array}{l}\text { Erfolg } \\
\text { Vitalitätsverlust }\end{array}$} & 1095 & $0.83[0.73 ; 0.92]$ & $0.87[0.81 ; 0.94]$ & $0.93[0.88 ; 0.98]$ \\
\hline & 1825 & $0.74[0.63 ; 0.86]$ & $0.83[0.75 ; 0.91]$ & $0.86[0.78 ; 0.94]$ \\
\hline & 2920 & $0.74[0.63 ; 0.86]$ & $0.7[0.59 ; 0.81]$ & $0.8[0.7 ; 0.9]$ \\
\hline & 3650 & $0.74[0.63 ; 0.86]$ & $0.7[0.59 ; 0.81]$ & $0.8[0.7 ; 0.9]$ \\
\hline \multirow{4}{*}{$\begin{array}{l}\text { Erfolg } \\
\text { Pfeilerfraktur }\end{array}$} & 1095 & $0.75[0.64 ; 0.85]$ & $0.88[0.81 ; 0.94]$ & $0.93[0.87 ; 0.98]$ \\
\hline & 1825 & $0.63[0.5 ; 0.75]$ & $0.79[0.7 ; 0.88]$ & $0.83[0.75 ; 0.92]$ \\
\hline & 2920 & $0.59[0.46 ; 0.73]$ & $0.71[0.6 ; 0.83]$ & $0.75[0.65 ; 0.86]$ \\
\hline & 3650 & $0.59[0.46 ; 0.73]$ & $0.71[0.6 ; 0.83]$ & $0.67[0.53 ; 0.8]$ \\
\hline \multirow{4}{*}{$\begin{array}{l}\text { Erfolg } \\
\text { Retentionsverlust }\end{array}$} & 1095 & $0.81[0.72 ; 0.9]$ & $0.85[0.78 ; 0.92]$ & $0.93[0.88 ; 0.98]$ \\
\hline & 1825 & 0.79 [0.69;0.89] & $0.81[0.73 ; 0.89]$ & $0.9[0.84 ; 0.96]$ \\
\hline & 2920 & $0.79[0.69 ; 0.89]$ & $0.7[0.57 ; 0.82]$ & $0.88[0.81 ; 0.95]$ \\
\hline & 3650 & $0.79[0.69 ; 0.89]$ & $0.7[0.57 ; 0.82]$ & $0.83[0.7 ; 0.96]$ \\
\hline \multirow{4}{*}{$\begin{array}{l}\text { Erfolg } \\
\text { Prothesenzahnfraktur }\end{array}$} & 1095 & $0.93[0.87 ; 0.99]$ & $0.85[0.77 ; 0.92]$ & $0.93[0.87 ; 0.98]$ \\
\hline & 1825 & $0.84[0.74 ; 0.95]$ & $0.82[0.74 ; 0.9]$ & $0.9[0.84 ; 0.96]$ \\
\hline & 2920 & $0.84[0.74 ; 0.95]$ & $0.82[0.74 ; 0.9]$ & $0.84[0.76 ; 0.93]$ \\
\hline & 3650 & $0.84[0.74 ; 0.95]$ & $0.74[0.58 ; 0.9]$ & $0.81[0.71 ; 0.91]$ \\
\hline \multirow{4}{*}{$\begin{array}{l}\text { Erfolg } \\
\text { Prothesenbasisfraktur }\end{array}$} & 1095 & $0.8[0.7 ; 0.9]$ & $0.87[0.8 ; 0.94]$ & $0.88[0.81 ; 0.95]$ \\
\hline & 1825 & $0.76[0.65 ; 0.88]$ & $0.79[0.71 ; 0.88]$ & $0.82[0.74 ; 0.91]$ \\
\hline & 2920 & $0.68[0.51 ; 0.85]$ & $0.71[0.59 ; 0.83]$ & $0.71[0.59 ; 0.83]$ \\
\hline & 3650 & $0.51[0.26 ; 0.76]$ & $0.59[0.42 ; 0.76]$ & $0.64[0.48 ; 0.8]$ \\
\hline
\end{tabular}

Tab. 12: Ereignis-Zeitanalysen (Kaplan-Meier) nach jeweils 3, 5, 8 und 10 Jahren mit 95\%-Konfidenzintervall abhängig von der Pfeileranzahl 


\begin{tabular}{|c|c|c|c|c|}
\hline Variable & Zeit [Tage] & $\begin{array}{l}\text { Festsitzender } \\
\text { Zahnersatz }\end{array}$ & Kombi-Zahnersatz & Totalprothese \\
\hline \multirow[t]{4}{*}{ Überleben } & 1095 & $0.79[0.64 ; 0.95]$ & $0.81[0.75 ; 0.88]$ & $0.75[0.65 ; 0.85]$ \\
\hline & 1825 & $0.66[0.47 ; 0.84]$ & $0.67[0.59 ; 0.75]$ & $0.49[0.37 ; 0.6]$ \\
\hline & 2920 & $0.48[0.27 ; 0.69]$ & $0.46[0.37 ; 0.56]$ & $0.14[0.05 ; 0.22]$ \\
\hline & 3650 & $0.38[0.17 ; 0.6]$ & $0.35[0.24 ; 0.47]$ & $0.07[0.01 ; 0.13]$ \\
\hline \multirow[t]{4}{*}{ Erfolg } & 1095 & $0.37[0.2 ; 0.55]$ & $0.28[0.2 ; 0.35]$ & $0.29[0.18 ; 0.39]$ \\
\hline & 1825 & $0.25[0.09 ; 0.4]$ & $0.13[0.08 ; 0.19]$ & $0.08[0.02 ; 0.13]$ \\
\hline & 2920 & $0.08[0 ; 0.17]$ & $0.04[0.01 ; 0.07]$ & NA [NA;NA] \\
\hline & 3650 & $0.04[0 ; 0.09]$ & $0.01[0 ; 0.03]$ & NA [NA;NA] \\
\hline \multirow{4}{*}{$\begin{array}{l}\text { Erfolg } \\
\text { Techn. Komplikation }\end{array}$} & 1095 & $0.67[0.49 ; 0.85]$ & $0.73[0.65 ; 0.8]$ & $0.76[0.66 ; 0.87]$ \\
\hline & 1825 & $0.51[0.31 ; 0.71]$ & $0.66[0.58 ; 0.74]$ & $0.61[0.47 ; 0.75]$ \\
\hline & 2920 & $0.43[0.23 ; 0.64]$ & $0.55[0.43 ; 0.66]$ & $0.55[0.39 ; 0.71]$ \\
\hline & 3650 & $0.43[0.23 ; 0.64]$ & $0.46[0.31 ; 0.61]$ & $0.55[0.39 ; 0.71]$ \\
\hline \multirow{4}{*}{$\begin{array}{l}\text { Erfolg } \\
\text { Biol. Kompliaktion }\end{array}$} & 1095 & $0.68[0.5 ; 0.85]$ & $0.53[0.45 ; 0.61]$ & $0.5[0.39 ; 0.62]$ \\
\hline & 1825 & $0.68[0.5 ; 0.85]$ & $0.43[0.35 ; 0.52]$ & $0.37[0.26 ; 0.49]$ \\
\hline & 2920 & $0.52[0.31 ; 0.74]$ & $0.39[0.3 ; 0.47]$ & $0.28[0.15 ; 0.4]$ \\
\hline & 3650 & $0.52[0.31 ; 0.74]$ & $0.39[0.3 ; 0.47]$ & $0.28[0.15 ; 0.4]$ \\
\hline \multirow{4}{*}{$\begin{array}{l}\text { Erfolg } \\
\text { Pfeilerverlust }\end{array}$} & 1095 & $0.72[0.55 ; 0.89]$ & $0.77[0.71 ; 0.84]$ & $0.7[0.6 ; 0.81]$ \\
\hline & 1825 & $0.5[0.31 ; 0.69]$ & $0.6[0.52 ; 0.69]$ & $0.44[0.32 ; 0.57]$ \\
\hline & 2920 & $0.34[0.16 ; 0.52]$ & $0.43[0.33 ; 0.52]$ & $0.12[0.03 ; 0.21]$ \\
\hline & 3650 & $0.34[0.16 ; 0.52]$ & $0.35[0.25 ; 0.46]$ & $0.12[0.03 ; 0.21]$ \\
\hline \multirow{4}{*}{$\begin{array}{l}\text { Erfolg } \\
\text { Sekundärkaries }\end{array}$} & 1095 & $0.96[0.88 ; 1]$ & $0.89[0.84 ; 0.94]$ & $0.9[0.83 ; 0.98]$ \\
\hline & 1825 & $0.89[0.76 ; 1]$ & $0.85[0.79 ; 0.91]$ & $0.81[0.7 ; 0.92]$ \\
\hline & 2920 & $0.89[0.76 ; 1]$ & $0.79[0.7 ; 0.88]$ & $0.68[0.53 ; 0.84]$ \\
\hline & 3650 & $0.89[0.76 ; 1]$ & $0.73[0.6 ; 0.85]$ & $0.55[0.32 ; 0.78]$ \\
\hline \multirow{4}{*}{$\begin{array}{l}\text { Erfolg } \\
\text { Vitalitätsverlust }\end{array}$} & 1095 & $0.96[0.89 ; 1]$ & $0.88[0.83 ; 0.94]$ & $0.84[0.75 ; 0.93]$ \\
\hline & 1825 & $0.84[0.68 ; 1]$ & $0.82[0.76 ; 0.88]$ & $0.8[0.7 ; 0.9]$ \\
\hline & 2920 & $0.84[0.68 ; 1]$ & $0.73[0.65 ; 0.82]$ & $0.69[0.54 ; 0.83]$ \\
\hline & 3650 & $0.84[0.68 ; 1]$ & $0.73[0.65 ; 0.82]$ & $0.69[0.54 ; 0.83]$ \\
\hline \multirow{4}{*}{$\begin{array}{l}\text { Erfolg } \\
\text { Pfeilerfraktur }\end{array}$} & 1095 & $0.92[0.81 ; 1]$ & $0.86[0.8 ; 0.91]$ & $0.83[0.74 ; 0.92]$ \\
\hline & 1825 & $0.75[0.57 ; 0.93]$ & $0.8[0.74 ; 0.87]$ & $0.64[0.51 ; 0.77]$ \\
\hline & 2920 & $0.75[0.57 ; 0.93]$ & $0.74[0.65 ; 0.82]$ & $0.54[0.39 ; 0.69]$ \\
\hline & 3650 & $0.5[0.21 ; 0.79]$ & $0.7[0.6 ; 0.8]$ & $0.54[0.39 ; 0.69]$ \\
\hline \multirow{4}{*}{$\begin{array}{l}\text { Erfolg } \\
\text { Retentionsverlust }\end{array}$} & 1095 & $0.83[0.69 ; 0.98]$ & $0.86[0.81 ; 0.92]$ & $0.88[0.8 ; 0.96]$ \\
\hline & 1825 & $0.78[0.62 ; 0.94]$ & $0.86[0.8 ; 0.91]$ & $0.8[0.69 ; 0.9]$ \\
\hline & 2920 & $0.7[0.51 ; 0.9]$ & $0.8[0.73 ; 0.88]$ & $0.8[0.69 ; 0.9]$ \\
\hline & 3650 & $0.7[0.51 ; 0.9]$ & $0.76[0.66 ; 0.87]$ & $0.8[0.69 ; 0.9]$ \\
\hline \multirow{4}{*}{$\begin{array}{l}\text { Erfolg } \\
\text { Prothesenzahnfraktur }\end{array}$} & 1095 & $0.8[0.64 ; 0.95]$ & $0.91[0.86 ; 0.96]$ & $0.9[0.83 ; 0.98]$ \\
\hline & 1825 & $0.8[0.64 ; 0.95]$ & $0.88[0.82 ; 0.93]$ & $0.83[0.72 ; 0.93]$ \\
\hline & 2920 & $0.71[0.51 ; 0.91]$ & $0.87[0.81 ; 0.92]$ & $0.78[0.65 ; 0.91]$ \\
\hline & 3650 & $0.71[0.51 ; 0.91]$ & $0.81[0.72 ; 0.9]$ & $0.78[0.65 ; 0.91]$ \\
\hline \multirow{4}{*}{$\begin{array}{l}\text { Erfolg } \\
\text { Prothesenbasisfraktur }\end{array}$} & 1095 & $0.67[0.49 ; 0.85]$ & $0.87[0.82 ; 0.93]$ & $0.89[0.82 ; 0.97]$ \\
\hline & 1825 & $0.61[0.42 ; 0.8]$ & $0.83[0.76 ; 0.89]$ & $0.8[0.68 ; 0.91]$ \\
\hline & 2920 & $0.46[0.24 ; 0.67]$ & $0.75[0.66 ; 0.84]$ & $0.59[0.38 ; 0.8]$ \\
\hline & 3650 & $0.46[0.24 ; 0.67]$ & $0.66[0.53 ; 0.79]$ & $0.44[0.2 ; 0.69]$ \\
\hline
\end{tabular}

Tab. 13: Ereignis-Zeitanalysen (Kaplan-Meier) nach jeweils 3, 5, 8 und 10 Jahren mit 95\%-Konfidenzintervall abhängig von der antagonistischen Versorgung 


\begin{tabular}{|c|c|c|c|c|c|}
\hline Variable & Gruppe & Koef & $\begin{array}{l}\text { Hazard } \\
\text { Ratio }\end{array}$ & $\begin{array}{l}\text { Konfidenz- } \\
\text { intervall }\end{array}$ & p \\
\hline \multirow[t]{4}{*}{ Überleben } & Anzahl Pfeiler1 & 1.26 & 3.54 & {$[2.22 ; 5.65]$} & $<0.001$ \\
\hline & Anzahl Pfeiler2 & 0.89 & 2.43 & {$[1.59 ; 3.71]$} & $<0.001$ \\
\hline & Antagonistische Versorgung Kombi.Zahnersatz & -0.03 & 0.97 & {$[0.54 ; 1.74]$} & 0.9154 \\
\hline & Antagonistische Versorgung Totalprothese & 0.4 & 1.49 & {$[0.8 ; 2.75]$} & 0.2054 \\
\hline \multirow[t]{4}{*}{ Erfolg } & Anzahl Pfeiler1 & 0.34 & 1.4 & {$[1 ; 1.97]$} & 0.0525 \\
\hline & Anzahl Pfeiler2 & 0.34 & 1.4 & {$[1.02 ; 1.92]$} & 0.0353 \\
\hline & Antagonistische Versorgung Kombi.Zahnersatz & 0.23 & 1.26 & {$[0.81 ; 1.94]$} & 0.3002 \\
\hline & Antagonistische Versorgung Totalprothese & 0.26 & 1.3 & {$[0.84 ; 2.01]$} & 0.2436 \\
\hline \multirow[t]{4}{*}{ Techn.Komplikation } & Anzahl Pfeiler1 & 0.47 & 1.6 & {$[0.97 ; 2.62]$} & 0.0633 \\
\hline & Anzahl Pfeiler2 & 0.26 & 1.3 & {$[0.8 ; 2.12]$} & 0.2953 \\
\hline & Antagonistische Versorgung Kombi.Zahnersatz & -0.38 & 0.68 & {$[0.36 ; 1.29]$} & 0.2397 \\
\hline & Antagonistische Versorgung Totalprothese & -0.48 & 0.62 & {$[0.31 ; 1.25]$} & 0.1818 \\
\hline \multirow[t]{4}{*}{ Biol.Komplikation } & Anzahl Pfeiler1 & 0.18 & 1.2 & {$[0.81 ; 1.77]$} & 0.3556 \\
\hline & Anzahl Pfeiler2 & 0.14 & 1.16 & {$[0.8 ; 1.66]$} & 0.4344 \\
\hline & Antagonistische Versorgung Kombi.Zahnersatz & 0.43 & 1.54 & {$[0.82 ; 2.89]$} & 0.1845 \\
\hline & Antagonistische Versorgung Totalprothese & 0.56 & 1.74 & {$[0.91 ; 3.33]$} & 0.093 \\
\hline \multirow[t]{4}{*}{ Pfeilerverlust } & Anzahl Pfeiler1 & 0.54 & 1.72 & {$[1.09 ; 2.73]$} & 0.0207 \\
\hline & Anzahl Pfeiler2 & 0.42 & 1.52 & {$[1 ; 2.32]$} & 0.0508 \\
\hline & Antagonistische Versorgung Kombi.Zahnersatz & -0.16 & 0.85 & {$[0.49 ; 1.46]$} & 0.5581 \\
\hline & Antagonistische Versorgung Totalprothese & 0.24 & 1.27 & {$[0.72 ; 2.25]$} & 0.4051 \\
\hline \multirow[t]{4}{*}{ Sekundärkaries } & Anzahl Pfeiler1 & -0.15 & 0.86 & {$[0.36 ; 2.07]$} & 0.7416 \\
\hline & Anzahl Pfeiler2 & 0.01 & 1.01 & {$[0.5 ; 2.04]$} & 0.9731 \\
\hline & Antagonistische Versorgung Kombi.Zahnersatz & 0.73 & 2.08 & {$[0.47 ; 9.15]$} & 0.3315 \\
\hline & Antagonistische Versorgung Totalprothese & 1.2 & 3.33 & {$[0.73 ; 15.05]$} & 0.1187 \\
\hline \multirow[t]{4}{*}{ Vitalitätsverlust } & Anzahl Pfeiler1 & 0.56 & 1.75 & {$[0.83 ; 3.69]$} & 0.1435 \\
\hline & Anzahl Pfeiler2 & 0.35 & 1.42 & {$[0.73 ; 2.77]$} & 0.3032 \\
\hline & Antagonistische Versorgung Kombi.Zahnersatz & 0.2 & 1.22 & {$[0.45 ; 3.28]$} & 0.6919 \\
\hline & Antagonistische Versorgung Totalprothese & 0.35 & 1.42 & {$[0.5 ; 4.08]$} & 0.5134 \\
\hline \multirow[t]{4}{*}{ Pfeilerfraktur } & Anzahl Pfeiler1 & 0.72 & 2.06 & {$[1.1 ; 3.88]$} & 0.0249 \\
\hline & Anzahl Pfeiler2 & 0.03 & 1.03 & {$[0.56 ; 1.9]$} & 0.9144 \\
\hline & Antagonistische Versorgung Kombi.Zahnersatz & -0.29 & 0.75 & {$[0.35 ; 1.63]$} & 0.4694 \\
\hline & Antagonistische Versorgung Totalprothese & 0.27 & 1.31 & {$[0.58 ; 2.95]$} & 0.5144 \\
\hline \multirow[t]{4}{*}{ Retentionsverlust } & Anzahl Pfeiler1 & 0.82 & 2.28 & {$[1.01 ; 5.14]$} & 0.0471 \\
\hline & Anzahl Pfeiler2 & 0.86 & 2.37 & {$[1.1 ; 5.09]$} & 0.0277 \\
\hline & Antagonistische Versorgung Kombi.Zahnersatz & -0.38 & 0.69 & {$[0.29 ; 1.62]$} & 0.3895 \\
\hline & Antagonistische Versorgung Totalprothese & -0.4 & 0.67 & {$[0.26 ; 1.74]$} & 0.4139 \\
\hline \multirow[t]{4}{*}{ Prothesenzahnfraktur } & Anzahl Pfeiler1 & 0.1 & 1.1 & {$[0.46 ; 2.61]$} & 0.828 \\
\hline & Anzahl Pfeiler2 & 0.44 & 1.55 & {$[0.73 ; 3.31]$} & 0.2555 \\
\hline & Antagonistische Versorgung Kombi.Zahnersatz & -0.74 & 0.48 & {$[0.19 ; 1.22]$} & 0.1221 \\
\hline & Antagonistische Versorgung Totalprothese & -0.5 & 0.61 & {$[0.21 ; 1.73]$} & 0.3507 \\
\hline \multirow[t]{4}{*}{ Prothesenbasisfraktur } & Anzahl Pfeiler1 & 0.35 & 1.41 & {$[0.7 ; 2.86]$} & 0.3359 \\
\hline & Anzahl Pfeiler2 & 0.24 & 1.27 & {$[0.7 ; 2.32]$} & 0.4256 \\
\hline & Antagonistische Versorgung Kombi.Zahnersatz & -1.11 & 0.33 & {$[0.16 ; 0.68]$} & 0.0026 \\
\hline & Antagonistische Versorgung Totalprothese & -0.78 & 0.46 & {$[0.2 ; 1.05]$} & 0.066 \\
\hline
\end{tabular}

Tab. 14: Risikoanalyse (Cox-Regression) bezüglich der Faktoren Pfeileranzahl und antagonistische Versorgung mit Modell-Koeffizient, Hazard Ratio und 95\%Konfidenzintervall; signifikante Unterschiede sind hervorgehoben 


\section{Literaturverzeichnis}

Adam P: Langzeituntersuchung über die Erfolgswahrscheinlichkeit von Teilprothesen mit Konuskronen nach K.-H. Körber. Med. Diss. Freiburg 1984

Bär C, Reiber T, Nitschke I (2009): Seniorenzahnmedizin in Deutschland: Status quo und Ziele der nahen und fernen Zukunft. Zahnärztl Mitt 5, 34-44

Becker H (1983): Wirkungsmechanismen der Haftung telekopierender Kronen. Zahnärztl Prax 34, 281

Behr M, Hofmann E, Rosentritt M, Lang R, Handel G (2000): Technical failure rate of double crown - retained removable partial dentures. Clin Oral Invest $\underline{4}, 87-90$

Bergmann B (1987): Periodontal reactions related to removable partial dentures: a literature review. J Prosthet Dent 58, 454-458

Bergman B, Hugoson A, Olsson CO (1977): Caries and periodontal status in patients fitted with removable partial dentures. J Clin Periodontol 4 , 134-146

Böttger H (1953): Die prothetische Versorgung des Lückengebisses mit Teleskopprothesen. Zahnärztl Rundsch 62, 512-518

Böttger H: Das Teleskopsystem in der zahnärztlichen Prothetik. Zahnärztliche Fortbildung, Heft 14. J. A. Barth, Leipzig 1961

Böttger H: Das Teleskopsystem in der zahnärztlichen Prothetik. Zahnärztliche Fortbildung, Heft 14. 4. Auflage; J. A. Barth, Leipzig 1973

Böttger H, Gründler H: Die Praxis des Teleskopsystems - das zahnärztliche und zahntechnische Vorgehen. 3. Auflage; Neuer Merkur, München 1970

Böttger H, Häupl K, Kirsten H: Zahnärztliche Prothetik - ein Lehrbuch für Studium und Praxis Band 2. 2. Auflage; J.A. Barth, Leipzig 1965

Coca J, Klimek K (2002): Vergleichende Langzeitstudie über das Verhalten von Pfeilerzähnen bei unterschiedlichen Versorgungsformen des reduzierten Lückengebisses im Unterkiefer. Zahn Prax 5, 252-254

Coca J, Lotzmann U, Pöggeler R (2000): Long-term experience with telescopically retained overdentures (double crown technique). Eur J Prosthodont Restor Dent 8 , 33-37

Diedrichs G (1990): Ist das Teleskopsystem noch zeitgemäß? Zahnärztl Welt 99 , 78-82

Dolder E, Wirz J: Die Steg-Gelenk-Prothese. Quintessenz Verlag, Berlin 1982 
Eisenburger M, Tschernitschek H (1998): Klinisch-technischer Vergleich zu Langzeitfolgen von klammerverankertem Zahnersatz und Teleskop-Prothesen. Dtsch Zahnärztl Z 53, 257-259

Eisenburger M, Gray G, Tschernitschek H (2000): Long-term results of telescopic crown retained dentures - a retrospective study. Eur J Prosthodont Restor Dent $\underline{8}$, 87-91

Ettinger RL, Jakobson J (1997): Denture treatment needs of an overdenture population. Int J Prosthodont 10, 355-365

Freesmeyer WB: Konstruktionselemente in der zahnärztlichen Prothetik: Planung und Einsatz unter funktionellen Gesichtspunkten. Carl Hanser, München/Wien 1987

Gernet W, Adam P, Reither W (1983): Nachuntersuchungen von Teilprothesen mit Konuskronen nach K.-H. Körber. Dtsch Zahnärztl Z 38, 998-1001

Graber G (1966): Teleskopkronen als Fixationsmittel unter schleimhautgetragener Prothesen. Schweiz Monatsschr Zahnheilk 76, 611-621

Hagener MW: Werkstoffwissenschatliche Untersuchungen zum Verschleiß von Telekopkronen. Med. Diss. Bonn 2006

Häupl K (1959): Das Teleskop im Dienste der Behandlung der Zahnlockerung. Österr Z Stomatol 56, 73-79

Häupl K, Reichborn-Kjennerud J: Moderne zahnärztliche Kronen- und Brückenarbeit. 1. Auflage; Hermann Meusser, Berlin 1929

Heners M, Walther W (1988): Pfeilerverteilung und starre Verblockung - eine klinische Langzeitstudie. Dtsch Zahnärztl Z $\underline{43}, 1122-1126$

Heners M, Walther W (1990): Die Prognose von Pfeilerzähnen bei stark reduziertem Restzahnbestand. Dtsch Zahnärztl Z $\underline{45}, 579-581$

Hofmann M (1966): Die Versorgung von Gebissen mit einzelstehenden Restzähnen mittels sog. Cover-Denture-Prothesen. Dtsch Zahnärztl Z 21, 478-482

Hofmann M: Die prothetische Versorgung bei einzelnen Restzähnen. In: Harndt E: Deutscher Zahnärztekalender. Jg. 35, Carl Hanser, München/Wien 1976, 32-52

Hofmann M, Ludwig P (1973): Die teleskopierende Totalprothese im stark reduzierten Lückengebiß. Dtsch Zahnärztl Z 28, 2-17

Jacoby W, Gasser F (1973): Nachträgliche Haltverbesserung von Teleskopkronen. Quintessenz 9, 59-62

Jüde HD, Kühl W, Rossbach A: Einführung in die zahnärztliche Prothetik.

2. überarbeitete Auflage; Deutscher Ärzte-Verlag, Köln-Lövenich 1979 
Jung T : Die prothetische Versorgung des stark reduzierten Restgebisses. In: Ketterl W: Deutscher Zahnärztekalender. Jg. 48, Carl Hanser, München/Wien 1989, 85-105

Kimmel K (2007): „Doppelkronen“ sind selbst nach 120 Jahren immer noch aktuell. DZW 16, 9

Kobes L (1969): Einzelne Restzähne und ihre Verwendung im Rahmen prothetischer Rehabilitationsmaßnahmen. Dtsch Zahnärztl Z 24, 401-409

Koeck B: Teilprothesen. 3. Auflage; Urban und Schwarzenberg, München 1996

Körber E (1954): Untersuchungen über die Beziehungen von Kaukraft zur Schleimhautresilienz unter Prothesen. Dtsch Zahnärztl Z 9, 348

Körber E (1980): Erfahrungen mit der Cover-Denture-Prothese. Stomatol DDR 30, 843-845

Körber E, Lehmann KM, Hoffmann U: Die Versorgung des stark reduzierten Lückengebisses mit gingival gelagerten Deckprothesen (teleskopierende Totalprothesen). In: Körber E: Die zahnärztlich - prothetische Versorgung des älteren Menschen.1. Auflage; Carl Hanser, München/Wien 1978, 148-159

Körber KH: Konuskronen - Teleskopkrone. Hüthig, Heidelberg 1974

Kothe A, Balkenhol M, Wickop H, Wöstmann B, Ferger P (2003): Orale Gesundheit und Lebensqualität vor und nach prothetischer Versorgung. Dtsch Zahnärztl Z 58, 603-605

Krämer A, Weber H (1990): Präzisionselemente in der Teilprothetik Teleskopierende Systeme. Zahnärztl Mitt 21, 2328-2331

Krennmair G, Krainhöfner M, Waldenberger O, Piehslinger E (2007): Dental implants as strategic supplementary abutments for implant-tooth-supported telescopic crownretained maxillary dentures: a retrospective follow-up study for up to 9 years. Int $\mathrm{J}$ Prosthodont 20, 617-622

Langer A (1981): Telescop retainers for removable partial dentures. J Prosthet Dent $\underline{45}, 37-43$

Lehmann KM, Gente M: Doppelkronen als Verankerung von abnehmbarem Zahnersatz. In: Ketterl W: Deutscher Zahnärztekalender. Jg. 47, Carl Hanser, München/Wien 1988, 106-120

Lehmann KM, Hellwig E, Wenz HJ: Zahnärztliche Propädeutik: Einführung in die Zahnheilkunde. 11. überarbeitete und erweiterte Auflage; Deutscher Zahnärzte Verlag, Köln 2008

Levin L (2008): Dealing with dental implant failures. J Appl Oral Sci 16, 171-175 
Ludwig P (1983):Grundlagen der Abstützung von herausnehmbarem Zahnersatz im Lückengebiss. Dtsch Zahnärztl Z 38, 967-974

Majewsky I von (1989): Zur Methodik des Resilienztelekopes im stark reduzierten Lückengebiss. Stomatol DDR 39, 499-501

Makowski A : Die häufigsten Reparaturen bei teleskopverankerten Prothesen. Med. Diss. Würzburg 2010

Meyer E (1983): Die Bewährung von Stegverbindungen, Teleskopkronen und Kugelknopfankern im stark reduzierten Gebiß. Dtsch Zahnärztl Z 38, 1011-1015

Mijiritsky E, Ormianer Z, Klinger A, Mardinger O (2005): Use of dental implants to improve unfavorable removable partial denture design. Compend Contin Educ Dent 26, $744-50$

Mock F: Bewährung teleskopverankerter Prothesen - eine klinische Langzeitstudie. Med. Diss. Saarbrücken 2005

Nitschke I, Müller F (2002): Die altersgerechte Praxis. BZB 4, 31-33

Pöggeler R: Klinische Nachuntersuchung von totalprothetischen Versorgungen mit Doppelkronen (Cover-Dentures). Med. Diss. Marburg 1995

Rehm H (1940): Grundsätzliche Betrachtungen zur partiellen abnehmbaren Prothese. Zahnärztl Rundsch $\underline{49}, 37$

Rehm H (1952): Über die Möglichkeiten der prothetischen Auswertung einzelner Frontzähne. Zahnärztl Welt $\underline{5}, 115$

Rehm H, Körber E, Körber KH (1962): Biophysikalischer Beitrag zur Problematik starr abgestützter Freiendprothesen. Dtsch Zahnärztl Z 17, 963-975

Rehmann P, Schmitt-Plank C, Balkenhol M, Wöstmann B, Ferger P (2007): Retrospektive Longitudinalstudie über die Bewährung von Freiendteleskopen mit ausschließlicher Verankerung auf den Unterkiefereckzähnen. Int Poster J Dent Oral Med 9, 362

Rehmann P, Weber A, Wöstmann B, Ferger P (2006): Klinische Bewährung von Zähnen, die zur Verankerung einer Teilprothese mit Teleskopkronen versorgt wurden. Dtsch Zahnärztl. Z 61, 662-666

Reither W (1978): Prothetische Konstruktionselemente als Schienungshilfsmittel zur Versorgung des parodontal insuffizienten Gebisses. Österr Z Stomatol DDR $\underline{75}, 99$

Sato M, Suzuki Y, Kurihara D, Shimpo H, Ohkubo C (2013): Effect of implant support on mandibular distal extension removable partial dentures: Relationship between denture supporting area and stress distribution. J Prosthodont Res 57,109-112 
Sauer G (1986): Mechanische Gesichtspunkte bei der Bewertung der Verbindungselemnte zwischen Restgebiss und Zahnersatz. Zahnärztl Welt 95 , 100-103

Schüth B: Die langfristige Bewährung von herausnehmbarem Zahnersatz. Med. Diss. Münster 1997

Schwanewede von H, Anderseck E (1985): Proteza Teleskopowa - Die Teleskopprothese im stark reduzierten Lückengebiss. Prot Stom 35, 166-170

Schwindling FS, Dittmann B, Rammelsberg P (2014): Double-crown-retained removable dental prostheses: a retrospective study of survival and complications. $J$ Prosthet Dent 112, 488-493

Stark H (1993): Untersuchungen über die Mundhygiene bei Trägern von Teleskopprothesen. Dtsch Zahnärztl Z 48, 570-572

Stark H: Klinische und werkstoffkundliche Untersuchungen zur Bewährung von Teleskopprothesen und zum Verschleißverhalten von Teleskopkronen. HänselHohenhausen, Egelsbach 1996

Stark H, Schrenker H (1998): Bewährung teleskopverankerter Prothesen - eine klinische Langzeitstudie. Dtsch Zahnärztl Z 53, 183-186

Stüttgen H: Das Reibungs- und Verschleißverhalten teleskopierender Prothesenanker. Quintessenz Verlag, Berlin 1985

Strub JR, Kern M, Türp JC, Witkowski S, Heydecke G, Wolfart S: Curriculum Prothetik, Band III. 4. überarbeitete und erweiterte Auflage; Quintessenz Verlag, Berlin 2011

Szentpetery V, Lautenschlager C, Setz JM (2012): Frictional telescopic crowns in severely reduced dentitions: a 5-year clinical outcome study. Int J Prosthodont 25, 217-220

Teubner E, Lorenzon A, Marinello CP (2007): Ästhetische und technische Aspekte in der konventionellen abnehmbaren Prothetik. Schweiz Monatsschr Zahnmed 117, 491-506

Vosbeck B: Nachuntersuchungen von Teleskopprothesenträgern. Med. Diss. Düsseldorf 1989

Wagner B, Kern M (2000): Clinical evalution of removable partial dentures 10 years after insertion: success rates, hygienic problems, and technical failures. Clin Oral Investig 4 , 74-80

Walther W (1990): Kronenfrakturen bei herausnehmbarem Zahnersatz. Dtsch Zahnärztl Z $\underline{45}$, 542-544 
Weber A: Überlebenszeitanalysen von teleskopverankerten Teilprothesen unter besonderer Berücksichtigung der Folgekosten. Med. Diss. Gießen 2005

Wenz HJ, Kern M (2007): Langzeitbewährung von Doppelkronen. Quintessenz Zahntech 33,1482-1494

Wenz HJ, Hertrampf K, Gente M, Lehmann K (1999): Langzeitverweildauer von Doppelkronen mit Spielpassung. Dtsch Zahnärztl Z 54, 655-657

Wenz HJ, Hertrampf K, Lehmann K (2001): Clinical longevity of removable partial dentures retained by telescopic crowns: Outcome oft the double crown with clearance fit. Int J Prosthodont 14, 207-213

Wupper H (1986): Zur Biomechanik verschiedener Verankerungssysteme Grundsätze zur Indikation von Geschieben, Stegen und Teleskopen. Zahnärztl Welt 95, 36

http://www.medizin-im-text.de/blog/2010/9859/hazard-ratio/, Zugriff am 28.11.2016 http://m.aerzteblatt.de/print/81171.htm, Zugriff am 28.11.2016 


\section{Danksagung}

Mein Dank gilt an dieser Stelle ganz besonders meinem Doktorvater, Herrn PD Dr. Matthias Rödiger, für die Überlassung des Themas sowie die freundliche, kontinuierliche Unterstützung und Beratung bei der Lösung aller anstehenden Fragen zur Fertigstellung dieser Arbeit.

Mein Dank gilt ebenfalls Herrn Prof. Dr. Nikolaus Gersdorff für die Ermöglichung dieser Dissertation.

Bei Herrn PD Dr. Sven Rinke bedanke ich mich herzlich für die freundliche Unterstützung während der Auswertung der Ergebnisse.

Frau Xenia Schulz aus der Abteilung Medizinische Statistik möchte ich für die statistische Auswertung danken. 


\section{Lebenslauf}

Am 18.08.1989 wurde ich, Vicky Wiedemann, als erste Tochter von Dr. med. Viola Wiedemann und Dr. med. Jens Kohlschreiber in Karl-Marx-Stadt, jetzt Chemnitz, geboren.

Von 1996 bis 2000 besuchte ich in Chemnitz die Grundschule Gablenz und wechselte 2000 auf das Johannes-Kepler-Gymnasium, an welchem ich 2008 meine Allgemeine Hochschulreife erlangte.

In der Zeit von 2008 bis 2009 leistete ich für 10 Monate ein Freiwilliges Soziales Jahr im DRK-Krankenhaus Chemnitz-Rabenstein ab.

Im Wintersemester 2009/2010 nahm ich das Studium der Zahnmedizin an der Georg-August-Universität Göttingen auf. Die naturwissenschaftliche Vorprüfung absolvierte ich im September 2010, die zahnärztliche Vorprüfung im März 2012. Im November 2014 legte ich die zahnärztliche Prüfung mit der Gesamtnote "sehr gut“ ab.

Im Januar 2015 begann ich meine Doktorarbeit in der Poliklinik für Zahnärztliche Prothetik im Zentrum Zahn-, Mund- und Kieferheilkunde der Medizinischen Fakultät der Universität Göttingen unter der Leitung von PD Dr. med. dent. M. Rödiger.

In der Zeit von September 2015 bis Ende September 2016 arbeitete ich als Assistenzzahnärztin in der Praxis Dr. Wostratzky in Leipzig.

Im Januar 2017 begann ich meine Weiterbildung zur Fachzahnärztin für Kieferorthopädie in der kieferorthopädischen Fachzahnarztpraxis Dr. Friedrich in Merseburg. Seit August 2017 bin ich als Weiterbildungsassistentin in der kieferorthopädischen Fachzahnarztpraxis Dr. Reich in Wurzen tätig. 